\title{
On Spectral Properties of the $L$ Operator in the Lax Pair of the sine-Gordon Equation
}

\author{
Thomas Kappeler and Yannick Widmer \\ To Professor Vladimir Aleksandrovich Marchenko \\ on the occasion of his ninety fifth birthday
}

We analyze the periodic spectrum of the $L$ operator in the Lax pair of the sine-Gordon equation in terms of the regularity of the potential.

Key words: sine-Gordon equation, sinh-Gordon equation, Lax pair, decay properties of gap lengths.

Mathematical Subject Classification 2010: 37K10, 35Q55.

\section{Introduction}

We consider the complex sine-Gordon equation

$$
u_{t t}-u_{x x}=-\sinh u, \quad x \in \mathbb{T}=\mathbb{R} / \mathbb{Z}, t \in \mathbb{R},
$$

where $u$ is assumed to be complex valued. In case $u$ is real valued, (1.1) is referred to as the sinh-Gordon equation and in case $u$ is purely imaginary, as real sineGordon equation. Equation (1.1) is a nonlinear perturbation of the (complex) Klein-Gordon equation $u_{t t}-u_{x x}=m u$ (with $m=-1$ ). It has wide ranging applications in geometry and quantum mechanics and has been extensively studied, although most of the work has been done for its version in light cone coordinates and hence does not apply to the periodic in space setup of (1.1). According to [2] (cf. also [8]), it admits a Lax pair. To describe it, note that (1.1) can be written as a system for $\left(u_{1}, u_{2}\right):=\left(u, u_{t}\right)$

$$
\left(\begin{array}{l}
u_{1} \\
u_{2}
\end{array}\right)_{t}=\left(\begin{array}{c}
u_{2} \\
u_{1 x x}-\sinh u_{1}
\end{array}\right) \text {. }
$$

In order to work with function spaces consisting of pairs of functions of equal regularity, we introduce

$$
(q, p):=\left(u_{1},-P^{-1} u_{2}\right) \in H_{c}^{1},
$$

where for any $s, H_{c}^{s}$ denotes the Sobolev space $H^{s}(\mathbb{T}, \mathbb{C}) \times H^{s}(\mathbb{T}, \mathbb{C})\left(\equiv H^{s}\left(\mathbb{T}, \mathbb{C}^{2}\right)\right)$ and $P$ the Fourier multiplier operator $P:=\sqrt{1-\partial_{x}^{2}}$. When expressed in these 
coordinates, equation (1.2) becomes

$$
\left(\begin{array}{c}
q_{t} \\
p_{t}
\end{array}\right)=\left(\begin{array}{c}
-P p \\
P q+P^{-1}(\sinh (q)-q)
\end{array}\right) .
$$

For any $v=(q, p) \in H_{c}^{1}$, define the following differential operators:

$$
Q(v)=Q_{1} \partial_{x}+Q_{0}(v), \quad K(v)=K_{1} \partial_{x}+K_{0}(v),
$$

where the coefficients $Q_{1}, Q_{0}, K_{1}, K_{0}$ are the $4 \times 4$ matrices given by

$$
\begin{aligned}
Q_{1} & =\left(\begin{array}{ll}
-J &
\end{array}\right), & Q_{0}(v) & =\left(\begin{array}{ll}
A(v) & B(v) \\
B(v) &
\end{array}\right), \\
K_{1} & =\left(\begin{array}{ll}
-I & \\
& I
\end{array}\right), & K_{0}(v) & =\left(\begin{array}{ll}
-2 B(v) J & -2 J B(v)
\end{array}\right),
\end{aligned}
$$

with $I, J, R, Z$ denoting the $2 \times 2$ matrices

$$
I=\left(\begin{array}{ll}
1 & \\
& 1
\end{array}\right), \quad J=\left(\begin{array}{rr} 
& 1 \\
-1 &
\end{array}\right), \quad R=\left(\begin{array}{ll}
\mathrm{i} & \\
& -\mathrm{i}
\end{array}\right), \quad Z=\left(\begin{array}{ll} 
& 1 \\
1 &
\end{array}\right)
$$

and

$$
A(v):=-\frac{1}{4}\left(P p+q_{x}\right) Z, \quad B(v):=\frac{1}{4}\left(\begin{array}{ll}
\exp (-q / 2) & \\
& \exp (q / 2)
\end{array}\right) .
$$

Here and in the sequel, we suppress matrix coefficients which vanish, so, e.g., $\left(\begin{array}{cc}-1 & 1\end{array}\right)$ stands for $\left(\begin{array}{cc}0 & 1 \\ -1 & 0\end{array}\right)$. One verifies that $t \mapsto v(t)$ is a solution of (1.1) iff $t \mapsto(Q(v(t)), K(v(t)))$ satisfies

$$
Q_{t}=[K, Q]
$$

The pair of operators $Q, K$ is referred to as Lax pair for the sine-Gordon equation and $Q$ as the corresponding $L$ operator. Note that (1.7) leads to an abundance of first integrals of (1.1): expressed in a somewhat informal way (i.e., without addressing issues of regularity) it follows from (1.7) that for any solution $t \mapsto$ $v(t)$ of (1.1), the periodic spectrum $\operatorname{spec}_{\text {per }} Q(v(t))$ of the operator $Q(v(t))$ is independent of $t$. Here for any $v \in H_{c}^{1}$, the periodic spectrum of $Q(v)$ is the spectrum of the operator $Q(v)$, considered with the Sobolev space $H^{1}\left(\mathbb{R} /(2 \mathbb{Z}), \mathbb{C}^{2}\right)$ of two periodic functions with values in $\mathbb{C}^{2}$ as its domain. Since for any $v \in H_{c}^{1}$, $\operatorname{spec}_{\text {per }} Q(v)$ is discrete this is saying that the periodic eigenvalues of $Q(v)$ are first integrals for the sine-Gordon equation.

Besides being of interest in its own right, the periodic spectrum of the operator $Q(v)$ and its associated spectral curve play a very important role in the construction of the normal form of the sine-Gordon equation. With this application in mind, the aim of this paper is to analyze $\operatorname{spec}_{\text {per }} Q(v)$. 
To state our main results, we first need to introduce some more notation. Define the domains $D_{0}:=\left\{z \in \mathbb{C}:\left|z-\frac{1}{4}\right|<\frac{1}{4 \pi}\right\}$ and for any $n \geq 1$,

$$
D_{n}:=\{\lambda \in \mathbb{C}:|\lambda-n \pi|<\pi / 3\}, \quad D_{-n}:=\left\{\lambda \in \mathbb{C}: \frac{1}{16 \lambda} \in D_{n}\right\} .
$$

Furthermore, let $B_{0}:=\{\lambda \in \mathbb{C}:|\lambda| \leq \pi / 2\}$ and for any $n \geq 1$

$$
B_{n}:=\{\lambda \in \mathbb{C}:|\lambda|<n \pi+\pi / 2\}, \quad B_{-n}:=\left\{\lambda \in \mathbb{C}:|\lambda| \leq \frac{1}{16(n \pi+\pi / 2)}\right\},
$$

and denote by $A_{n}$ the open annulus $A_{n}:=B_{n} \backslash B_{-n}$. By the Counting Lemma (cf. Lemma 3.11 in Section 3) for any potential in $H_{c}^{1}$, there exist a neighborhood $U$ in $H_{c}^{1}$ and an integer $N \geq 1$, such that for any $v \in U$ and any $n>N$, the operator $Q(v)$ has exactly two periodic eigenvalues in each of the domains $D_{n}$, $-D_{n}, D_{-n}$, and $-D_{-n}$ and exactly $4+8 N$ roots in the annulus $A_{N}$, counted with their multiplicities. There are no further eigenvalues. We denote the eigenvalues in $D_{k},|k| \geq N$ by $\lambda_{k}^{+}, \lambda_{k}^{-}$and list them in the following order, $\left[\left|\lambda_{k}^{-}\right|<\left|\lambda_{k}^{+}\right|\right]$ or $\left[\left|\lambda_{k}^{-}\right|=\left|\lambda_{k}^{+}\right|\right.$and $\left.\operatorname{Im} \lambda_{k}^{-} \leq \operatorname{Im} \lambda_{k}^{+}\right]$. By symmetry, for any $k \in \mathbb{Z}$ with $|k|>$ $N$, the two eigenvalues in $-D_{k}$ are given by $-\lambda_{k}^{+}$and $-\lambda_{k}^{-}$. By a slight abuse of terminology, we refer to the difference $\lambda_{k}^{+}-\lambda_{k}^{-}$as the $k^{\prime}$ th gap length.

Theorem 1.1. For any $v \in H_{c}^{s+1}$ with $s \geq 0$ and for $N \geq 1$ given as above, the following asymptotic estimates of the gap lengths of the periodic spectrum $Q(v)$ hold: there exists $C>0$ so that

$$
\begin{gathered}
\sum_{n>N}\langle n\rangle^{2 s}\left|\lambda_{n}^{+}-\lambda_{n}^{-}\right|^{2} \leq C, \\
\sum_{n>N}\langle n\rangle^{2 s}\left|\left(16 \lambda_{-n}^{+}\right)^{-1}-\left(16 \lambda_{-n}^{-}\right)^{-1}\right|^{2} \leq C .
\end{gathered}
$$

Furthermore, $N$ and $C$ can be chosen locally uniformly with respect to $v$.

To state the second result, we first need to introduce some more notation. Let $v \in H_{c}^{1}$ and let $N \geq 1$ be as above. Then for any $N^{\prime} \geq N, v \in H_{c}^{1}$ is said to be a right [left] sided $N^{\prime}$-gap potential if

$$
\forall n>N^{\prime} \quad \lambda_{n}^{+}=\lambda_{n}^{-} \quad\left[\forall n>N^{\prime} \quad \lambda_{-n}^{+}=\lambda_{-n}^{-}\right] .
$$

It is said to be a right [left] sided finite gap potential if it is a right [left] sided $N^{\prime}$-gap potential for some $N^{\prime} \geq N$. For any $s \geq 1$, denote by $L F G_{c}^{s}$ and $R F G_{c}^{s}$ the following subsets of $H_{c}^{s}$ :

$$
L F G_{c}^{s}:=\left\{v \in H_{c}^{s}: v \text { left sided finite gap potential }\right\}
$$

and

$$
R F G_{c}^{s}:=\left\{v \in H_{c}^{s}: v \text { right sided finite gap potential }\right\} .
$$

Theorem 1.2. For any real number $s$ with $s \geq 1$, the sets $L F G_{c}^{s}$ and $R F G_{c}^{s}$ are dense in $H_{c}^{s}$. 


\section{Comments:}

(i) The presented results are part of a larger project of constructing normal coordinates, also referred to as Birkhoff coordinates, for the sine-Gordon equation. When expressed in these coordinates, the sine-Gordon equation is in normal form. It allows to solve it by quadrature and to obtain KAM type theorems for (small) perturbations of it. Previously, such a program has been carried out for the KdV equation (cf. [5] and references therein) and the defocusing NLS equation (cf. [3] and references therein). To carry this out for the sine-Gordon equation turns out to be more challenging since in this case, the spectral analysis of the L-operator (cf. 1.4) is more complicated (cf. Sections 2 and 3).

(ii) More detailed versions of Theorem 1.1 and Theorem 1.2 are stated in Theorem 4.10 and Theorem 4.15 in Section 4. Their proofs are based on a Lyapunov-Schmidt decomposition developed in previous work for the Hill and Zakharov-Shabat operators - see $[1,4,6,9]$ and references therein. Our work confirms that this method of proof can be applied in a wide variety of cases, but that the estimates needed can become quite involved.

(iii) Many questions remain open which we plan to address in future work. We expect that the developed techniques allow to obtain also asymptotic estimates for $\mu_{n}-\left(\lambda_{n}^{+}+\lambda_{n}^{-}\right) / 2$, similar to the ones in (1.8) and (1.9) obtained for the gap lengths. Here $\mu_{n}$ denote the Dirichlet eigenvalues of $Q(v)$ (cf. Section 3). Furthermore, we expect that $\lambda_{n}^{+}, \lambda_{n}^{-}$admit asymptotic expansions in $n^{-1}$ of the type Marchenko proved in seminal work for the periodic eigenvalues of Hill's operator and of the Zhakarov-Shabat operator (cf. [7]) and that (1.8), (1.9) characterize the regularity of the potential $v$ in case $v$ is real or purely complex valued. It means that in such a case, any $v \in H_{c}^{1}$ satisfying (1.8) and (1.9) is actually in $H_{c}^{s+1}$. Although the statement of Theorem 1.2 is sufficient for the applications we have in mind we expect that with the methods developed, it can be shown that for any $s \geq 1$, the intersection $L F G_{c}^{s} \cap R F G_{c}^{s}$ is dense in $H_{c}^{s}$.

Related work: As already mentioned, the sine-Gordon equation has been extensively studied, although most of the work has been done for its version in light cone coordinates. We only mention that in the periodic in space setup, important contributions can be found in [8] where parts of the material discussed in Sections 2 and 3 are presented and the spectral curve associated with $Q(v)$ is studied.

Organisation: In Section 2, we record the results needed on the fundamental solution of the operator $Q(v)$, the main purpose being to introduce notation and to state the results in the form needed later. In Section 3, we study the asymptotics of the periodic, the Dirichlet, and the Neumann eigenvalues of the operator $Q(v)$ for $v \in H_{c}^{1}$. They provide the setup for the proofs of Theorem 1.1 and Theorem 1.2 in Section 4. Although the material in these two sections is by and large known (cf., e.g., [8]), for the convenience of the reader, we included the 
proofs of the stated results. In Section 4 we prove Theorem 1.1 and Theorem 1.2.

\section{Fundamental solution}

In this section we study the fundamental solution of the differential operator $Q=Q(v)$ defined in (1.4) where $v=(q, p) \in H_{c}^{1}$. The results obtained will be used in particular in Section 3 in order to analyze the periodic and the Dirichlet spectrum of $Q$. Note that $Q F=0$ if and only if $F=0$ and for any given $\lambda \in$ $\mathbb{C}^{*}=\mathbb{C} \backslash\{0\}$, a function $F$ in $H_{\text {loc }}^{1}\left(\mathbb{R}, \mathbb{C}^{4}\right)$ is a solution of

$$
Q F=\lambda F
$$

if and only if $F=\left(f, \lambda^{-1} B f\right)$ and $f$ satisfies the following first order ODE:

$$
-J \partial_{x} f+\left(A+B^{2} / \lambda\right) f=\lambda f .
$$

Here and in the sequel we often write $\partial_{x} f$ for $f_{x}$. Let $M=M(x, \lambda, v) \in \mathbb{C}^{2 \times 2}$ be the fundamental solution for equation (2.2), meaning that

$$
\left(-J \partial_{x}+A(x)+B^{2}(x) / \lambda\right) M(x, \lambda, v)=\lambda M(x, \lambda, v), \quad M(0, \lambda, v)=I d .
$$

Clearly one has

$$
\partial_{x} M=J\left(\lambda-A-B^{2} / \lambda\right) M,
$$

or, taking the definition (1.6) into account,

$$
\partial_{x} M(x, \lambda, v)=J\left(\lambda+\frac{1}{4}\left(P p(x)+q_{x}(x)\right)\left(\begin{array}{ll} 
& 1 \\
1 &
\end{array}\right)-\frac{1}{16 \lambda}\left(\begin{array}{ll}
e^{-q(x)} & \\
& e^{q(x)}
\end{array}\right)\right) M .
$$

It is convenient to also use the matrix valued function $\mathcal{M}$ instead of $M$ where

$$
\mathcal{M}(x, \lambda, v)=T M(x, \lambda, v) T^{-1}, \quad T=\left(\begin{array}{cc}
1 & \mathrm{i} \\
1 & -\mathrm{i}
\end{array}\right), \quad T^{-1}=\frac{1}{2}\left(\begin{array}{cc}
1 & 1 \\
-\mathrm{i} & \mathrm{i}
\end{array}\right) .
$$

Introduce

$$
\mathcal{Q}=\left(\begin{array}{ll}
T & \\
& T
\end{array}\right) Q\left(\begin{array}{cc}
T^{-1} & \\
& T^{-1}
\end{array}\right)=\mathcal{Q}_{1} \partial_{x}+\mathcal{Q}_{0}(v)
$$

where

$$
\begin{gathered}
\mathcal{Q}_{1}=\left(\begin{array}{l}
R \\
\end{array}\right), \quad \mathcal{Q}_{0}(v)=\left(\begin{array}{cc}
\mathcal{A}(v) & \mathcal{B}(v) \\
\mathcal{B}(v) &
\end{array}\right), \\
\mathcal{A}(v)=T A(v) T^{-1}=-\frac{\mathrm{i}}{4}\left(P p+q_{x}\right) J \\
\mathcal{B}(v)=T B(v) T^{-1}=\frac{1}{4}\left(\begin{array}{cc}
\cosh (q / 2) & -\sinh (q / 2) \\
-\sinh (q / 2) & \cosh (q / 2)
\end{array}\right),
\end{gathered}
$$

and hence

$$
\mathcal{B}(v)^{2}=T B(v)^{2} T^{-1}=\frac{1}{16}\left(\begin{array}{cc}
\cosh (q) & -\sinh (q) \\
-\sinh (q) & \cosh (q)
\end{array}\right)
$$


An element $F$ in $H_{\text {loc }}^{1}\left(\mathbb{R}, \mathbb{C}^{4}\right)$ is a solution of $Q F=\lambda F$ iff $\mathcal{Q} T F=\lambda T F$. Furthermore $\mathcal{F}$ on $H_{\text {loc }}^{1}\left(\mathbb{R}, \mathbb{C}^{4}\right)$ is a solution of

$$
\mathcal{Q F}=\lambda \mathcal{F}
$$

iff $\mathcal{F}=\left(\mathcal{F}_{1}, \lambda^{-1} \mathcal{B F}_{1}\right)$ and

$$
\partial_{x} \mathcal{F}_{1}=-R\left(\lambda-\mathcal{A}-\mathcal{B}^{2} / \lambda\right) \mathcal{F}_{1}
$$

Hence $\mathcal{M}$ satisfies

$$
\partial_{x} \mathcal{M}=-R\left(\lambda-\mathcal{A}-\mathcal{B}^{2} / \lambda\right) \mathcal{M}, \quad \mathcal{M}(0, \lambda, v)=I .
$$

First we discuss symmetries of the fundamental solution $M$ needed in the sequel.

Proposition 2.1 (Symmetries). For any $(x, \lambda, v) \in[0, \infty) \times \mathbb{C}^{*} \times H_{c}^{1}$

(i) (Reflection of $\lambda$ )

$$
M(x,-\lambda, v)=-R M(x, \lambda, v) R \quad \text { and } \quad \mathcal{M}(x,-\lambda, v)=-Z \mathcal{M}(x, \lambda, v) Z .
$$

(ii) (Reciprocity of $\lambda$ )

$$
M\left(x, \frac{1}{16 \lambda}, q, p\right)=-R e^{-\mathrm{i} R q(x) / 2} M(x, \lambda,-q, p) e^{\mathrm{i} R q(0) / 2} R
$$

or

$$
M(x, \lambda,-q, p)=-R e^{\mathrm{i} R q(x) / 2} M\left(x, \frac{1}{16 \lambda}, q, p\right) e^{-\mathrm{i} R q(0) / 2} R
$$

(iii) (Conjugation)

$$
M(x, \bar{\lambda}, \bar{v})=\overline{M(x, \lambda, v)} \quad \text { and } \quad \mathcal{M}(x, \bar{\lambda}, \bar{v})=Z \overline{\mathcal{M}(x, \lambda, v)} Z .
$$

(iv) (Reflection of $v$ )

$$
M(x, \lambda,-v)=J M(x, \lambda, v) J^{-1} \quad \text { and } \quad \mathcal{M}(x, \lambda,-v)=R \mathcal{M}(x, \lambda, v) R^{-1} .
$$

Proof. (i) First note that $M(x,-\lambda, v)$ and $-R M(x, \lambda, v) R$ coincide at $x=0$. Hence it suffices to show that they satisfy both the same first order differential equation. By (2.3) $M(x,-\lambda, v)$ satisfies the equation

$$
\partial_{x} M(x,-\lambda, v)=J\left(-\lambda+\frac{1}{4}\left(P p(x)+q_{x}(x)\right) Z+\frac{1}{16 \lambda} e^{\mathrm{i} R q(x)}\right) M(x,-\lambda, v)
$$

and thus

$$
\partial_{x}(-R M(x, \lambda, v) R)
$$




$$
=-R J\left(\lambda+\frac{1}{4}\left(P p(x)+q_{x}(x)\right) Z-\frac{1}{16 \lambda} e^{\mathrm{i} R q(x)}\right) M(x, \lambda, v) R
$$

Since $R J=-J R, R Z=-Z R$ and $R e^{\mathrm{i} R q}=e^{\mathrm{i} R q} R$, one concludes that $-R M(x, \lambda, v) R$ satisfies the same equation as $M(x,-\lambda, v)$,

$$
\begin{aligned}
& \partial_{x}(-R M(x, \lambda, v) R) \\
& \quad=J\left(-\lambda+\frac{1}{4}\left(P p(x)+q_{x}(x)\right) Z+\frac{1}{16 \lambda} e^{\mathrm{i} R q(x)}\right)(-R M(x, \lambda, v) R)
\end{aligned}
$$

as claimed. Concerning the second identity of item (i), note that

$$
Z=-\mathrm{i} T R T^{-1}
$$

and hence $-Z \mathcal{M} Z=-Z T M T^{-1} Z=T R M R T^{-1}$ which yields the claimed identity for $\mathcal{M}(x,-\lambda, v)$.

(ii) Again note that $M\left(x,-\frac{1}{16 \lambda}, q, p\right)$ and $e^{-\mathrm{i} R q(x) / 2} M(x, \lambda,-q, p) e^{\mathrm{i} R q(0) / 2}$ coincide at $x=0$. Hence it suffices to show that they satisfy both the same first order differential equation. By $(2.3), M\left(x,-\frac{1}{16 \lambda}, q, p\right)$ satisfies

$$
\begin{aligned}
\partial_{x}(x, & \left.-\frac{1}{16 \lambda}, q, p\right) \\
& =J\left(-\frac{1}{16 \lambda}+\frac{1}{4}\left(P p(x)+q_{x}(x)\right) Z+\lambda e^{\mathrm{i} R q(x)}\right) M\left(x,-\frac{1}{16 \lambda}, q, p\right) .
\end{aligned}
$$

On the other hand, $\partial_{x}\left(e^{-\mathrm{i} R q(x) / 2} M(x, \lambda,-q, p)\right)$ equals

$$
\begin{aligned}
e^{-\mathrm{i} R q(x) / 2} J\left(\lambda+\frac{1}{4}\left(P p(x)-q_{x}(x)\right) Z\right. & \left.-\frac{1}{16 \lambda} e^{-\mathrm{i} R q(x)}\right) M(x, \lambda,-q, p) \\
& -\frac{1}{2} q_{x}(x) \mathrm{i} R e^{-\mathrm{i} R q(x) / 2} M(x, \lambda,-q, p) .
\end{aligned}
$$

Since $e^{-\mathrm{i} R q / 2} J=J e^{\mathrm{i} R q / 2}, e^{\mathrm{i} R q / 2} Z=Z e^{-\mathrm{i} R q / 2}$ and $\mathrm{i} R=-J Z$, one gets

$$
\begin{aligned}
& \partial_{x}\left(e^{-\mathrm{i} R q(x) / 2} M(x, \lambda,-q, p)\right) \\
& =J\left(\lambda e^{\mathrm{i} R q(x)}+\frac{1}{4}\left(P p(x)+q_{x}(x)\right) Z-\frac{1}{16 \lambda}\right) e^{-\mathrm{i} R q(x) / 2} M(x, \lambda,-q, p) .
\end{aligned}
$$

By the latter identity and (2.14), one sees that $M\left(x,-\frac{1}{16 \lambda}, q, p\right)$ and $e^{-\mathrm{i} R q(x) / 2} M(x, \lambda,-q, p) e^{\mathrm{i} R q(0) / 2}$ satisfy the same differential equation and hence must coincide. The first identity of item (ii) then follows from (i). The second identity of (ii) is obtained using the first one.

(iii) Take the complex conjugate of (2.3), $\partial_{x} \overline{M(x, \lambda, v)}=J(\bar{\lambda}-\overline{A(v)}-$

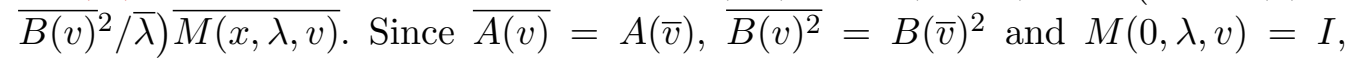
one concludes that $\overline{M(x, \lambda, v)}=M(x, \bar{\lambda}, \bar{v})$. The second identity of item (iii) then also follows since by $(2.4), \overline{T^{-1}}=T^{-1} Z$. 
(iv) Note that $M(x, \lambda,-v)$ satisfies

$$
\partial_{x} M(x, \lambda,-v)=J\left(\lambda-\frac{1}{4}\left(P p(x)+q_{x}(x)\right) Z-\frac{1}{16 \lambda} e^{-\mathrm{i} R q(x)}\right) M(x, \lambda,-v)
$$

while $\partial_{x} J M(x, \lambda, v)=J J\left(\lambda+\frac{1}{4}\left(P p(x)+q_{x}(x)\right) Z-\frac{1}{16 \lambda} e^{\mathrm{i} R q(x)}\right) M(x, \lambda, v)$. The first claimed identity then follows from $J Z=-Z J$ and $J e^{\mathrm{i} R q}=e^{-\mathrm{i} R q} J$ and the second one from $T J T^{-1}=-R$.

To obtain estimates for the fundamental solution of $Q$, we write (2.12) as an integral equation and represent its solution as a series. Here and in the sequel we will use the Euclidean norm for vectors in $\mathbb{C}^{2}$ and the induced operator norm for matrices. We write $M=\left(\begin{array}{ll}m_{1} & m_{2} \\ m_{3} & m_{4}\end{array}\right)$ where $m_{j}=m_{j}(x, \lambda, v)$. The norm $|M|$ of $M$ induced by the Hermitian norm on $\mathbb{C}^{2}$ can be bounded as follows:

$$
|M| \leq 2 \max \left\{\left|m_{1}\right|+\left|m_{2}\right|,\left|m_{3}\right|+\left|m_{4}\right|\right\} .
$$

Let us first compute $\mathcal{M}(x, \lambda, 0)$. By (2.12), it satisfies $\partial_{x} \mathcal{M}=-R\left(\lambda-\frac{1}{16 \lambda}\right) \mathcal{M}$. For $\lambda \in \mathbb{C}^{*}$, let

$$
\mathcal{E}_{\omega}(x):=e^{-R \omega x}=\left(\begin{array}{cc}
e^{-\mathrm{i} \omega x} & \\
& e^{\mathrm{i} \omega x}
\end{array}\right), \quad \omega \equiv \omega(\lambda)=\lambda-\frac{1}{16 \lambda} .
$$

Then $\partial_{x} \mathcal{E}_{\omega}=-R \omega \mathcal{E}_{\omega}$ and hence $\mathcal{E}_{\omega}(x)=\mathcal{M}(x, \lambda, 0)$. Note that $\omega(\lambda)=0$ iff $\lambda=$ $\pm \frac{1}{4}$ and

$$
\omega\left(\frac{1}{16 \lambda}\right)=\omega(-\lambda), \quad \omega(-\lambda)=-\omega(\lambda), \quad \forall \lambda \in \mathbb{C}^{*} .
$$

For $v \in H_{c}^{1}, \mathcal{M}(x)-\mathcal{E}_{\omega}(x)=\mathcal{E}_{\omega}(0) \mathcal{M}(x)-\mathcal{E}_{\omega}(x) \mathcal{M}(0)$ satisfies the integral equation

$$
\begin{aligned}
\mathcal{M}(x)-\mathcal{E}_{\omega}(x) & =\int_{0}^{x} \partial_{s}\left(\mathcal{E}_{\omega}(x-s) \mathcal{M}(s)\right) \mathrm{d} s \\
& =\int_{0}^{x} R \omega \mathcal{E}_{\omega}(x-s) \mathcal{M}(s)-\mathcal{E}_{\omega}(x-s) R\left(\lambda-\mathcal{A}-\mathcal{B}^{2} / \lambda\right) \mathcal{M}(s) \mathrm{d} s \\
& =\int_{0}^{x} \mathcal{E}_{\omega}(x-s) R\left(\omega-\lambda+\mathcal{A}+\mathcal{B}^{2} / \lambda\right) \mathcal{M}(s) \mathrm{d} s
\end{aligned}
$$

or

$$
\mathcal{M}(x)-\mathcal{E}_{\omega}(x)=\int_{0}^{x} \mathcal{E}_{\omega}(x-s) \Phi_{\lambda}(s) \mathcal{M}(s) \mathrm{d} s,
$$

where by (2.7)-(2.9)

$$
\begin{aligned}
\Phi_{\lambda}(s) & =R\left(\omega-\lambda+\mathcal{A}+\mathcal{B}^{2} / \lambda\right) \\
& =R\left(\frac{1}{16 \lambda}\left(\begin{array}{cc}
\cosh (q)-1 & -\sinh (q) \\
-\sinh (q) & \cosh (q)-1
\end{array}\right)-\frac{\mathrm{i}}{4}\left(P p+q_{x}\right) J\right) .
\end{aligned}
$$


To investigate the regularity of the fundamental solution we use equation (2.20) to find a series representation for $\mathcal{M}$. Let $\mathcal{M}_{0}(x)=\mathcal{E}_{\omega}(x)$ and define $\mathcal{M}_{n+1}(x)$ inductively by

$$
\mathcal{M}_{n+1}(x)=\int_{0}^{x} \mathcal{E}_{\omega}(x-s) \Phi_{\lambda}(s) \mathcal{M}_{n}(s) \mathrm{d} s .
$$

Using that $\mathcal{E}_{\omega}(x-s)=\mathcal{E}_{\omega}(x) \mathcal{E}_{\omega}(-s)$, one obtains the following identity for $\mathcal{M}_{n+1}$,

$$
\begin{aligned}
\mathcal{M}_{n+1}(x)= & \int_{0}^{x} \mathcal{E}_{\omega}(x-s) \Phi_{\lambda}(s) \mathcal{M}_{n}(s) \mathrm{d} s \\
= & \mathcal{E}_{\omega}(x) \int_{0 \leq x_{1} \leq \cdots \leq x_{n} \leq x} \underset{\mathcal{E}_{\omega}\left(-x_{n}\right) \Phi_{\lambda}\left(x_{n}\right) \mathcal{E}_{\omega}\left(x_{n}\right) \cdots}{ } \\
& \times \mathcal{E}_{\omega}\left(-x_{1}\right) \Phi_{\lambda}\left(x_{1}\right) \mathcal{E}_{\omega}\left(x_{1}\right) \mathrm{d} x_{1} \cdots \mathrm{d} x_{n} .
\end{aligned}
$$

As usual, we denote by $\|q\|_{s}$ the $H^{s}(\mathbb{T}, \mathbb{C})$-norm of $q=\sum_{k \in \mathbb{Z}} \widehat{q}_{2 k} e^{2 \pi \mathrm{i} k x}$

$$
\|q\|_{s}=\left(\sum_{k \in \mathbb{Z}}\left|\widehat{q}_{2 k}\right|^{2}\langle 2 k\rangle^{2 s}\right)^{1 / 2}, \quad\langle 2 k\rangle=\sqrt{1+(2 k \pi)^{2}}
$$

and write $\|v\|_{s}=\|q\|_{s}+\|p\|_{s}$ as well as $\|v\|_{0} \equiv\|v\|_{L^{2}}=\|q\|_{L^{2}}+\|p\|_{L^{2}}$. Note that for any $q \in H_{\mathbb{C}}^{1}$, one has

$$
\|q\|_{L^{\infty}} \leq 2\|q\|_{1}
$$

Theorem 2.2 (Regularity of the fundamental solution). The series $\mathcal{M}(x)=$ $\sum_{n=0}^{\infty} \mathcal{M}_{n}(x)$, with $\mathcal{M}_{n}(x)$ given by $(2.21)$, converges in $\mathbb{C}^{2 \times 2}$ absolutely, uniformly on bounded closed subsets of $[0, \infty) \times \mathbb{C}^{*} \times H_{c}^{1} . \mathcal{M}$ is continuous in $x, \lambda, v$ and analytic in $v=(q, p)$ and $\lambda$ as a map with values in the Banach space $C\left([0,2], \mathbb{C}^{2 \times 2}\right)$ of continuous functions with values in $\mathbb{C}^{2 \times 2}$, endowed with the supremum norm. It is the unique solution of $(2.12), \partial_{x} \mathcal{M}=$ $-R\left(\lambda-\mathcal{A}-\mathcal{B}^{2} / \lambda\right) \mathcal{M}, \mathcal{M}(0, \lambda, v)=I$, implying that $\mathcal{M}$ and $\partial_{x} \mathcal{M}$ are analytic in $v$ and $\lambda$ as maps with values in $L^{2}\left([0,2], \mathbb{C}^{2 \times 2}\right)$. Furthermore $\mathcal{M}$ satisfies the following estimate for any $0 \leq x \leq 2, \lambda \in \mathbb{C}^{*}, v \in H_{c}^{1}$,

$$
|\mathcal{M}(x, \lambda, v)| \leq e^{|\operatorname{Im} \omega| x} \exp \left(e^{2|\operatorname{Im} \omega| x}\left(\frac{x}{|\lambda|} e^{2\|q\|_{1}}+\sqrt{x}\|v\|_{1}\right)\right) .
$$

Proof. Clearly, one has $\left|\mathcal{M}_{0}(x)\right|=\left|\mathcal{E}_{\omega}(x)\right|=e^{|\operatorname{Im} \omega| x}$. To estimate $\mathcal{M}_{n+1}$ for $n \geq 0$, we first need to estimate $F(x, \lambda, v):=\int_{0}^{x} \mathcal{E}_{\omega}(-s) \Phi_{\lambda}(s) \mathcal{E}_{\omega}(s) \mathrm{d} s$. Use the bound (2.17) of the matrix norm and the identity

$$
\begin{aligned}
\mathcal{E}_{\omega}(-s) \Phi_{\lambda}(s) \mathcal{E}_{\omega}(s)= & \frac{\mathrm{i}}{16 \lambda}\left(\begin{array}{cc}
\cosh (q(s))-1 & -\sinh (q(s)) e^{2 \mathrm{i} \omega s} \\
\sinh (q(s)) e^{-2 \mathrm{i} \omega s} & 1-\cosh (q(s))
\end{array}\right) \\
& +\frac{1}{4}\left(P p(s)+q_{x}(s)\right)\left(\begin{array}{cc}
e^{-2 \mathrm{i} \omega s} & e^{2 \mathrm{i} \omega s}
\end{array}\right)
\end{aligned}
$$

to conclude that

$$
|F(x, \lambda, v)| \leq \frac{1}{|\lambda|} \max _{ \pm} \int_{0}^{x}\left(|\cosh (q(s))-1|+\left|e^{ \pm 2 \mathrm{i} \omega s} \sinh (q(s))\right|\right) \mathrm{d} s
$$




$$
+\max _{ \pm} \int_{0}^{x}\left|\left(P p(s)+q_{x}(s)\right) e^{ \pm 2 \mathrm{i} \omega s}\right| \mathrm{d} s
$$

Since

$$
|\cosh (q)-1| \leq \sum_{n \geq 1} \frac{1}{(2 n) !}|q|^{2 n} \quad \text { and } \quad|\sinh (q)| \leq \sum_{n \geq 0} \frac{1}{(2 n+1) !}|q|^{2 n+1},
$$

one has

$$
|\cosh (q)-1|+|\sinh (q)| \leq e^{|q|}-1 .
$$

Using that

$$
\left|e^{ \pm 2 \mathrm{i} \omega s}\right| \leq e^{2|\operatorname{Im} \omega| s} \quad \text { and } \quad e^{2|\operatorname{Im} \omega| s} \geq 1,
$$

it then follows that

$$
|F(x, \lambda, v)| \leq \int_{0}^{x} e^{2|\operatorname{Im} \omega| s}\left(e^{|q(s)|} \frac{1}{|\lambda|}+\left|P p(s)+q_{x}(s)\right|\right) \mathrm{d} s .
$$

Combining (2.23) with the estimate

$$
\begin{aligned}
\left\|e^{2|\operatorname{Im} \omega| s}\left|P p+q_{x}\right|\right\|_{L^{1}([0, x])} & \leq \sqrt{x} e^{2|\operatorname{Im} \omega| x}\left\|P p+q_{x}\right\|_{L^{2}([0, x])} \\
& \leq \sqrt{x} e^{2|\operatorname{Im} \omega| x}\|v\|_{1},
\end{aligned}
$$

one gets that for any $x \geq 0$,

$$
|F(x, \lambda, v)| \leq e^{2|\operatorname{Im} \omega| x}\left(\frac{x}{|\lambda|} e^{2\|q\|_{1}}+\sqrt{x}\|v\|_{1}\right) .
$$

Since the matrix norm is sub-multiplicative, one obtains

$$
\begin{aligned}
\left|\mathcal{M}_{n+1}(x)\right| & \leq e^{|\operatorname{Im} \omega| x} \int_{0 \leq x_{1} \leq \cdots \leq x_{n} \leq x} \prod_{k=1}^{n}\left|\mathcal{E}_{\omega}\left(-x_{k}\right) \Phi_{\lambda}\left(x_{k}\right) \mathcal{E}_{\omega}\left(x_{k}\right)\right| \mathrm{d} x_{n} \mathrm{~d} x_{n-1} \cdots \mathrm{d} x_{1} \\
& \leq \frac{e^{|\operatorname{Im} \omega| x}}{n !}|F(x, \lambda, v)|^{n} .
\end{aligned}
$$

Hence by (2.26), the series converges normally as claimed and one has

$$
|\mathcal{M}(x, \lambda, v)| \leq e^{|\operatorname{Im} \omega| x} \exp \left(e^{2|\operatorname{Im} \omega| x}\left(\frac{x}{|\lambda|} e^{2\|q\|_{1}}+\sqrt{x}\|v\|_{1}\right)\right), \quad x \geq 0 .
$$

Since for any given $x \geq 0, \mathcal{E}_{\omega}(x)$ and $\Phi_{\lambda}(x)$ are analytic in $(\lambda, v) \in \mathbb{C}^{*} \times H_{c}^{1}$ and continuous in $(x, \lambda, v) \in[0, \infty) \times \mathbb{C}^{*} \times H_{c}^{1}$ so is $\mathcal{M}_{n+1}$ for any $n \geq 0$ by the definition (2.21) and hence $\mathcal{M}=\sum_{n \geq 0} \mathcal{M}_{n}$ in view of the normal convergence of the series. It then follows that $\mathcal{M}$ is analytic as a map of $v$ and $\lambda$ with values in $C\left([0,2], \mathbb{C}^{2 \times 2}\right)$. Finally substituting the series into (2.21) and using that by the normal convergence of the series, sum and integral commute, one gets (2.20). Since $\mathcal{M}(x)$ and $\mathcal{E}_{\omega}(x)$ are continuous, (2.12) holds in the $L^{2}$ sense. It then follows that $\mathcal{M}$ and $\partial_{x} \mathcal{M}$ are continuous in $v$ and $\lambda$ as maps with values in $L^{2}\left([0,2], \mathbb{C}^{2 \times 2}\right)$. 
From Theorem 2.2 we derive the following bounds for $M=T^{-1} \mathcal{M} T$.

Corollary 2.3. $M$ is continuous in $x, v, \lambda$ and for each fixed $x$, it is analytic in $v$ and $\lambda$. It is the unique solution of (2.3), implying that $M$ and $\partial_{x} M$ are continuous in $v$ and $\lambda$ as maps with values in $L^{2}\left([0,2], \mathbb{C}^{2 \times 2}\right)$. Furthermore $M$ satisfies the following estimates for any $0 \leq x \leq 2, \lambda \in \mathbb{C}^{*}, v \in H_{c}^{1}$,

$$
|M(x, \lambda, v)| \leq e^{|\operatorname{Im} \omega| x} \exp \left(e^{2|\operatorname{Im} \omega| x}\left(\frac{x}{|\lambda|} e^{2\|q\|_{1}}+\sqrt{x}\|v\|_{1}\right)\right)
$$

and

$$
\left|M\left(x, \frac{1}{16 \lambda}, v\right)\right| \leq e^{2\|q\|_{1}+|\operatorname{Im} \omega| x} \exp \left(e^{2|\operatorname{Im} \omega| x}\left(\frac{x}{|\lambda|} e^{2\|q\|_{1}}+\sqrt{x}\|v\|_{1}\right)\right) .
$$

Proof. Since $M=T^{-1} \mathcal{M}$, the regularity statements follow from Theorem 2.2. Furthermore, as $\frac{\mathrm{i}}{\sqrt{2}} T$ is unitary one gets

$$
|M(x, \lambda, v)| \leq e^{|\operatorname{Im} \omega| x} \exp \left(e^{2|\operatorname{Im} \omega| x}\left(\frac{x}{|\lambda|} e^{2\|q\|_{1}}+\sqrt{x}\|v\|_{1}\right)\right), \quad x \geq 0 .
$$

Since $\omega\left(\frac{1}{16 \lambda}\right)=\omega(-\lambda)=-\omega(\lambda)$ and by Proposition 2.1 (ii),

$$
M\left(x, \frac{1}{16 \lambda}, q, p\right)=-R e^{-\mathrm{i} R q(x) / 2} M(x, \lambda,-q, p) e^{\mathrm{i} R q(0) / 2} R,
$$

the latter estimate yields

$$
\begin{aligned}
\left|M\left(x, \frac{1}{16 \lambda}, q, p\right)\right| & \leq e^{2\|q\|_{1}}|M(x, \lambda,-q, p)| \\
& \leq e^{2\|q\|_{1}+|\operatorname{Im} \omega| x} \exp \left(e^{2|\operatorname{Im} \omega| x}\left(\frac{x}{|\lambda|} e^{2\|q\|_{1}}+\sqrt{x}\|v\|_{1}\right)\right) .
\end{aligned}
$$

Next we prove that $M$ is compact in $v$, uniformly on closed bounded sets of $(x, \lambda) \in[0, \infty) \times \mathbb{C}^{*}$.

Definition 2.4. We call a map from a subset $U$ of a Hilbert space $H$ into some Banach space compact if it maps sequences in $U$ which converge weakly in $H$, to strongly convergent sequences.

Proposition 2.5. For any sequence $\left(v_{n}\right)_{n \geq 1}$ in $H_{c}^{1}$ which converges weakly to an element $v_{*}$ in $H_{c}^{1}$, one has $\left|M\left(x, \lambda, v_{n}\right)-M\left(x, \lambda, v_{*}\right)\right| \rightarrow 0$ as $n \rightarrow \infty$, uniformly on closed bounded subsets of $[0, \infty) \times \mathbb{C}^{*}$.

Proof. In view of $M=T^{-1} \mathcal{M} T$, it is enough to prove that $\mathcal{M}$ is compact in $v$ uniformly on closed bounded sets of $(x, \lambda) \in[0, \infty) \times \mathbb{C}^{*}$. In view of the uniform convergence of the series $\sum_{m=0}^{\infty} \mathcal{M}_{m}(x)$, it suffices to prove the statement for each term $\mathcal{M}_{m}$. For $M_{0}=E_{\omega}$ the statement is obviously true, since this term 
does not depend on $v$. Now by induction assume that the statement is true for $\mathcal{M}_{m}$, and let $\left(v_{n}\right)_{n \geq 0}$ converge weakly to $v_{*}$ in $H_{c}^{1}$. By equation (2.21) we have

$$
\mathcal{M}_{m+1}\left(x, \lambda, v_{n}\right)=\int_{0}^{x} \mathcal{E}_{\omega}(x-s) \Phi\left(s, \lambda, v_{n}\right) \mathcal{M}_{m}\left(s, \lambda, v_{n}\right) \mathrm{d} s .
$$

By the induction hypothesis $\left|\mathcal{M}_{m}\left(x, \lambda, v_{n}\right)-\mathcal{M}_{m}\left(x, \lambda, v_{*}\right)\right| \rightarrow 0$ uniformly on closed bounded subsets of $[0, \infty) \times \mathbb{C}^{*}$. Furthermore, the weak convergence of $v_{n}$ in $H_{c}^{1}$ implies that $P p_{n}+\left(q_{n}\right)_{x} \rightarrow P p_{*}+\left(q_{*}\right)_{x}$ in $L^{2}([0, x])$ and $q_{n} \rightarrow q_{*}, p_{n} \rightarrow$ $p_{*}$ in $L^{\infty}(\mathbb{T})$. It then follows that $\cosh \left(q_{n}\right) \rightarrow \cosh \left(q_{*}\right), \sinh \left(q_{n}\right) \rightarrow \sinh \left(q^{*}\right)$ in $L^{2}([0, x])$, yielding that $\mathcal{E}_{\omega}(x-\cdot) \Phi\left(\cdot, \lambda, v_{n}\right)$ weakly converges to $\mathcal{E}_{\omega}(x-\cdot) \Phi\left(\cdot, \lambda, v_{*}\right)$ in $L^{2}([0, x])$, uniformly on bounded subsets of $[0, \infty) \times \mathbb{C}^{*}$. Hence the sequence $\int_{0}^{x} \mathcal{E}_{\omega}(x-s) \Phi\left(s, \lambda, v_{n}\right) \mathcal{M}_{m}\left(s, \lambda, v_{n}\right) \mathrm{d} s$ converges uniformly on closed bounded subsets of $[0, \infty) \times \mathbb{C}^{*}$ to

$$
\int_{0}^{x} \mathcal{E}_{\omega}(x-s) \Phi\left(s, \lambda, v_{*}\right) \mathcal{M}_{m}\left(s, \lambda, v_{*}\right) \mathrm{d} s=\mathcal{M}_{m+1}\left(x, \lambda, v_{*}\right) .
$$

Since $J\left(\lambda-A-B^{2} / \lambda\right)$ is traceless,

$$
\operatorname{det} M(x, \lambda, v)=1 \text {. }
$$

Hence for any $(x, \lambda, v) \in[0, \infty] \times \mathbb{C}^{*} \times H_{c}^{1}, M(x, \lambda, v)=\left(\begin{array}{ll}m_{1} & m_{2} \\ m_{3} & m_{4}\end{array}\right)$ is invertible and $M^{-1}(x, \lambda, v)=\left(\begin{array}{cc}m_{4} & -m_{2} \\ -m_{3} & m_{1}\end{array}\right)$ with

$$
\left|M^{-1}(x, \lambda, v)\right| \leq 2 \max \left(\left|m_{4}\right|+\left|m_{2}\right|,\left|m_{3}\right|+\left|m_{1}\right|\right) .
$$

Proposition 2.6. The $\lambda$-derivative $\dot{M}$ of $M$ is given by

$$
\dot{M}(x)=M(x) \int_{0}^{x} M^{-1}(s) J\left(1+B^{2} / \lambda^{2}\right) M(s) \mathrm{d} s .
$$

In particular, for any $x \geq 0, \dot{M}(x, \lambda, v)$ is analytic on $\mathbb{C}^{*} \times H_{c}^{1}$ and on any closed bounded subset of $[0, \infty) \times \mathbb{C}^{*} \times H_{c}^{1}$, it is compact and bounded.

Proof. Taking the $\lambda$-derivative on both sides of equation (2.3) one sees that the $\lambda$-derivative $\dot{M}$ of $M$ fulfills

$$
\partial_{x} \dot{M}=J\left(\lambda-A-B^{2} / \lambda\right) \dot{M}+J\left(1+B^{2} / \lambda^{2}\right) M
$$

with $\dot{M}(0, \lambda, v)(0)=0$. The solution of this inhomogeneous linear equation for $\dot{M}$ is then given by

$$
\dot{M}(x)=M(x) \int_{0}^{x} M^{-1}(s) J\left(1+B^{2} / \lambda^{2}\right) M(s) \mathrm{d} s .
$$

From this formula and the properties established for $M$, the remaining statements for $\dot{M}$ follow. 
Next we establish bounds for the difference of the fundamental solution $M$ with $M(x, \lambda, 0)=E_{\omega}(x)$ for $|\lambda|$ large and small. First we need to establish the following auxiliary result.

Lemma 2.7. For any $v \in H_{c}^{1}$ and any $(x, \lambda) \in[0,1] \times \mathbb{C}^{*}$,

$$
F(x, \lambda, v)=\int_{0}^{x} \mathcal{E}_{\omega}(-s) \Phi_{\lambda}(s) \mathcal{E}_{\omega}(s) \mathrm{d} s
$$

satisfies

$\left|\mathcal{E}_{\omega}(x) F(x, \lambda, v)\right| \leq \frac{1}{|\lambda|} e^{|\operatorname{Im} \omega(\lambda)| x} e^{2\|q\|_{1}}+\max _{ \pm}\left|\int_{0}^{x}\left(P p(s)+q_{x}(s)\right) e^{ \pm \mathrm{i} \omega(\lambda)(x-2 s)} \mathrm{d} s\right|$.

Proof. Multiply (2.24) by $\mathcal{E}_{\omega}(x)$ to get

$$
\begin{aligned}
\mathcal{E}_{\omega}(x) F(x, \lambda, v)= & \int_{0}^{x} \frac{\mathrm{i}}{16 \lambda}\left(\begin{array}{cc}
e^{-\mathrm{i} \omega x}(\cosh (q(s))-1) & -\sinh (q(s)) e^{-\mathrm{i} \omega(x-2 s)} \\
\sinh (q(s)) e^{\mathrm{i} \omega(x-2 s)} & e^{\mathrm{i} \omega x}(1-\cosh (q(s)))
\end{array}\right) \\
& +\frac{1}{4}\left(P p(s)+q_{x}(s)\right)\left(\begin{array}{cc}
e^{\mathrm{i} \omega(x-2 s)} & e^{-\mathrm{i} \omega(x-2 s)}
\end{array}\right) \mathrm{d} s
\end{aligned}
$$

Hence

$$
\begin{aligned}
\left|\mathcal{E}_{\omega}(x) F(x, \lambda, v)\right| \leq & \int_{0}^{x} \frac{1}{|\lambda|} e^{|\operatorname{Im} \omega| x}(|\cosh (q(s))-1|+|\sinh (q(s))|) \mathrm{d} x \\
& +\left|\int_{0}^{x}\left(P p(s)+q_{x}(s)\right) e^{|\operatorname{Im} \omega|(x-2 s)} \mathrm{d} s\right|
\end{aligned}
$$

Using that $|\cosh (q(s))-1|+|\sinh (q(s))| \leq e^{2\|q\|_{1}}$ yields the claim.

For $(x, \lambda, v) \in[0,1] \times \mathbb{C}^{*} \times H_{c}^{1}$, let

$$
\widehat{\mathcal{M}}(x, \lambda, v):=\mathcal{M}(x, \lambda, v)-\mathcal{E}_{\omega}(x) .
$$

Lemma 2.8. On $[0,1] \times H_{c}^{1}$ for all $\lambda \in \mathbb{C}$ with $|\lambda| \geq 1 / 4$,

$$
\begin{aligned}
|\widehat{\mathcal{M}}(x, \lambda, v)| \leq & \left|\mathcal{E}_{\omega}(x) F(x, \lambda, v)\right| \\
& +C_{v} e^{|\operatorname{Im} \omega| x}\left(\int_{0}^{x} e^{-2|\operatorname{Im} \omega| s}\left|\mathcal{E}_{\omega}(s) F(s, \lambda, v)\right|^{2} \mathrm{~d} s\right)^{1 / 2},
\end{aligned}
$$

where $C_{v}=c e^{c}$ and $c=\|v\|_{1}+e^{2\|q\|_{1}}$.

Proof. By (2.20),

$$
\widehat{\mathcal{M}}(x, \lambda, v)=\int_{0}^{x} \mathcal{E}_{\omega}(x-s) \Phi_{\lambda}(s) \mathcal{M}(s) \mathrm{d} s
$$

satisfies

$$
\widehat{\mathcal{M}}(x, \lambda, v)=\int_{0}^{x} \mathcal{E}_{\omega}(x-s) \Phi_{\lambda}(s) \mathcal{E}_{\omega}(s) \mathrm{d} s+\int_{0}^{x} \mathcal{E}_{\omega}(x-s) \Phi_{\lambda}(s) \widehat{\mathcal{M}}(s) \mathrm{d} s,
$$


yielding

$$
\widehat{\mathcal{M}}(x, \lambda, v)=\mathcal{E}_{\omega}(x) F(x, \lambda, v)+\int_{0}^{x} \mathcal{E}_{\omega}(x-s) \Phi_{\lambda}(s) \widehat{\mathcal{M}}(s) \mathrm{d} s .
$$

Clearly,

$$
\left|\mathcal{E}_{\omega}(x-s) \Phi_{\lambda}(s) \widehat{\mathcal{M}}(s)\right| \leq e^{|\operatorname{Im} \omega|(x-s)}\left|\Phi_{\lambda}(s)\right||\widehat{\mathcal{M}}(s)| .
$$

It is convenient to introduce the following weighted norm for a $x$-dependent $2 \times 2$ matrix

$$
|A(x)|_{\omega}:=e^{-|\operatorname{Im} \omega| x}|A(x)| .
$$

Multiplying both sides of (2.31) by $e^{-|\operatorname{Im} \omega| x}$ one obtains the following estimate

$$
|\widehat{\mathcal{M}}(x)|_{\omega} \leq\left|\mathcal{E}_{\omega}(x) F(x, \lambda, v)\right|_{\omega}+\int_{0}^{x}\left|\Phi_{\lambda}(s)\right||\widehat{\mathcal{M}}(s)|_{\omega} \mathrm{d} s
$$

and hence by Gronwall's inequality and the estimate

$$
|\Phi(s)| \leq\left(\left|P p(s)+q_{x}(s)\right|+\frac{1}{|\lambda|} e^{|q(s)|}\right)=: b(s, \lambda)
$$

we get

$$
\begin{aligned}
|\widehat{\mathcal{M}}(x)|_{\omega} \leq & \left|\mathcal{E}_{\omega}(x) F(x, \lambda, v)\right|_{\omega} \\
& +\int_{0}^{x}\left|\mathcal{E}_{\omega}(s) F(s, \lambda, v)\right|_{\omega} b(s, \lambda) \exp \left(\int_{s}^{x} b(r, \lambda) \mathrm{d} r\right) \mathrm{d} s .
\end{aligned}
$$

Arguing as in (2.25) with $x=1$, one gets

$$
\int_{0}^{1} b(r, \lambda) \mathrm{d} r \leq\|v\|_{1}+\frac{1}{|\lambda|} e^{2\|q\|_{1}}
$$

An application of the Cauchy-Schwarz inequality then yields the claim.

We now use Lemma 2.8 to derive estimates for $\mathcal{M}$ and $\dot{\mathcal{M}}$ from those of $F$.

Theorem 2.9. $\mathcal{M}$ and $\dot{\mathcal{M}}$ have the following asymptotics:

(i) For $|\lambda| \rightarrow \infty$, locally uniformly on $[0,1] \times H_{c}^{1}$,

$$
\begin{aligned}
& \mathcal{M}(x, \lambda, v)=\mathcal{E}_{\omega(\lambda)}(x)+o\left(e^{|\operatorname{Im} \omega(\lambda)| x}\right), \\
& \dot{\mathcal{M}}(x, \lambda, v)=\dot{\mathcal{E}}_{\omega(\lambda)}(x)+o\left(e^{|\operatorname{Im} \omega(\lambda)| x}\right),
\end{aligned}
$$

where $\dot{\mathcal{E}}_{\omega(\lambda)}(x)=-\mathrm{i} \dot{\omega}(\lambda) R \mathcal{E}_{\omega(\lambda)}(x)$ and $\dot{\omega}(\lambda)=1+\frac{1}{16 \lambda^{2}}$.

(ii) For $|\lambda| \rightarrow \infty$, uniformly on $[0,1]$ and on bounded subsets of $H_{c}^{2}$,

$$
\begin{aligned}
& \mathcal{M}(x, \lambda, v)=\mathcal{E}_{\omega(\lambda)}(x)+O\left(e^{|\operatorname{Im} \omega(\lambda)| x} /|\omega(\lambda)|\right), \\
& \dot{\mathcal{M}}(x, \lambda, v)=\dot{\mathcal{E}}_{\omega(\lambda)}(x)+O\left(e^{|\operatorname{Im} \omega(\lambda)| x} /|\omega(\lambda)|\right) .
\end{aligned}
$$


Proof. (i) In view of Lemma 2.8 it remains to prove an appropriate asymptotic estimate for $\left|\mathcal{E}_{\lambda}(x) F(x, \lambda, v)\right|$. By Lemma 2.7

$$
\begin{aligned}
\left|\mathcal{E}_{\omega(\lambda)}(x) F(x, \lambda, v)\right| \leq & \frac{1}{|\lambda|} e^{|\operatorname{Im} \omega(\lambda)| x} e^{2\|q\|_{1}} \\
& +\max _{ \pm}\left|\int_{0}^{x}\left(P p(s)+q_{x}(s)\right) e^{ \pm \mathrm{i} \omega(\lambda)(x-2 s)} \mathrm{d} s\right| .
\end{aligned}
$$

For arbitrary $\epsilon>0$ there is $\omega_{\epsilon}>0$, depending locally uniformly on $v \in H_{c}^{1}$, such that for any $\lambda \in \mathbb{C}^{*}$ with $|\omega(\lambda)|>\omega_{\epsilon}$ and $0 \leq x \leq 1$ one has (cf. Lemma D.1 in [3])

$$
\left|\int_{0}^{x}\left(P p(s)+q_{x}(s)\right) e^{ \pm \mathrm{i} \omega(x-2 s)} \mathrm{d} s\right| \leq \epsilon e^{|\operatorname{Im} \omega| x},
$$

yielding the stated asymptotics of $\mathcal{M}$. The claimed asymptotics for $\dot{\mathcal{M}}$ is obtained by applying Cauchy's estimate to the $\lambda$-derivative of $\widehat{\mathcal{M}}$.

(ii) In case $v \in H_{c}^{2}$,

$$
\int_{0}^{x}\left(P p(s)+q_{x}(s)\right) e^{ \pm \mathrm{i} \omega(x-2 s)} \mathrm{d} s
$$

can be integrated by parts. Using that for $\omega \equiv \omega(\lambda) \neq 0$,

$$
e^{ \pm \mathrm{i} \omega(x-2 s)}=\frac{-1}{ \pm 2 \mathrm{i} \omega} \frac{\mathrm{d}}{\mathrm{d} s} e^{ \pm \mathrm{i} \omega(x-2 s)}
$$

one gets

$$
\begin{aligned}
\int_{0}^{x}(P p(s) & \left.+q_{x}(s)\right) e^{ \pm \mathrm{i} \omega(x-2 s)} \mathrm{d} s=-\frac{1}{ \pm 2 \mathrm{i} \omega}\left(\left(P p(x)+q_{x}(x)\right) e^{\mp \mathrm{i} \omega x}\right. \\
& \left.+\left(P p(0)+q_{x}(0)\right) e^{ \pm \mathrm{i} \omega x}\right)-\int_{0}^{x}\left(P p_{x}(s)+q_{x x}(s)\right) e^{ \pm \mathrm{i} \omega(x-2 s)} \mathrm{d} s .
\end{aligned}
$$

Since by (2.23), $\left\|q_{x}\right\|_{L^{\infty}} \leq 2\|q\|_{2}$ and

$$
\|P p\|_{L^{\infty}} \leq 2\|P p\|_{1}=2\left(\sum_{k}\langle 2 k\rangle^{2}\left|\langle 2 k\rangle \widehat{p}_{2 k}\right|^{2}\right)^{1 / 2}=2\|p\|_{2}
$$

one gets for any $0 \leq x \leq 1,|\omega(\lambda)|>0$ and $v \in H_{c}^{2}$

$$
\begin{aligned}
\left|\mathcal{E}_{\omega(\lambda)}(x) F(x, \lambda, v)\right| \leq & \frac{1}{|\lambda|} e^{|\operatorname{Im} \omega(\lambda)| x} e^{2\|q\|_{1}} \\
& +\frac{1}{2|\omega(\lambda)|}\left(2\|v\|_{2} e^{|\operatorname{Im} \omega(\lambda)| x}+2\|v\|_{2} e^{|\operatorname{Im} \omega(\lambda)| x}\right) .
\end{aligned}
$$

The claimed asymptotics for $\dot{\mathcal{M}}$ is once more obtained by applying Cauchy's estimate to the $\lambda$-derivative of $\widehat{\mathcal{M}}$.

Theorem 2.10. For bi-infinite sequences of complex numbers $\left(\zeta_{n}\right)_{n} \subset \mathbb{C}^{*}$ with $\left|\zeta_{n}\right| \geq \frac{1}{4}$, the following holds: 
(i) If $\zeta_{n}=n \pi+O(1)$, then

$$
\mathcal{M}\left(x, \zeta_{n}, v\right)=\mathcal{E}_{\omega\left(\zeta_{n}\right)}(x)+\ell_{n}^{2} \quad \text { and } \quad \dot{\mathcal{M}}\left(x, \zeta_{n}, v\right)=\dot{\mathcal{E}}_{\omega\left(\zeta_{n}\right)}(x)+\ell_{n}^{2},
$$

where $\mathcal{E}_{\omega\left(\zeta_{n}\right)}(x)=e^{-R \omega\left(\zeta_{n}\right) x}$ and $\dot{\omega}\left(\zeta_{n}\right)=1+\frac{1}{16 \zeta_{n}^{2}}$, implying that

$$
\dot{\mathcal{E}}_{\omega\left(\zeta_{n}\right)}(x)=-x \dot{\omega}\left(\zeta_{n}\right) R e^{-R \omega\left(\zeta_{n}\right) x}=-x R \mathcal{E}_{\omega\left(\zeta_{n}\right)}(x)+\ell_{n}^{1} .
$$

These estimates hold uniformly on $[0,1]$, on bounded subsets of $H_{c}^{1}$ and on subsets of sequences $\left(\zeta_{n}\right)_{n}$ where $\left(\omega\left(\zeta_{n}\right)-n \pi\right)_{n}$ is bounded in $\ell_{\mathbb{C}}^{\infty}$. In more detail, e.g., the first estimate means that for any bounded subset $V \subset H_{c}^{1}$ and any subset $B$ of sequences $\left(\zeta_{n}\right)_{n} \subset \mathbb{C}^{*}$, with $\left(\omega\left(\zeta_{n}\right)-n \pi\right)_{n}$ bounded in $\ell_{\mathbb{C}}^{\infty}$, there exists $C>0$ so that

$$
\sup _{0 \leq x \leq 1} \sum_{n \in \mathbb{Z}}\left|\mathcal{M}\left(x, \zeta_{n}, v\right)-\mathcal{E}_{\omega\left(\zeta_{n}\right)}(x)\right|^{2} \leq C, \quad \text { for any } v \in V \text { and }\left(\zeta_{n}\right)_{n} \in B .
$$

(ii) If $\zeta_{n}=n \pi+\ell_{n}^{2}$, then

$$
\mathcal{M}\left(x, \zeta_{n}, v\right)=\mathcal{E}_{n \pi}(x)+\ell_{n}^{2} \quad \text { and } \quad \dot{\mathcal{M}}\left(x, \zeta_{n}, v\right)=-x R \mathcal{E}_{n \pi}(x)+\ell_{n}^{2} .
$$

These estimates hold uniformly on $[0,1]$, on bounded subsets of $H_{c}^{1}$, and on subsets of sequences $\left(\zeta_{n}\right)_{n}$ where $\left(\omega\left(\zeta_{n}\right)-n \pi\right)_{n}$ is bounded in $\ell_{\mathbb{C}}^{2}$.

Proof. (i) By Lemma 2.8, on $[0,1] \times H_{c}^{1}$ for any $|\lambda| \geq 1 / 4$

$$
\left|\mathcal{M}(x, \lambda, v)-\mathcal{E}_{\omega}(x)\right| \leq O\left(e^{|\operatorname{Im} \omega|}\left\|\mathcal{E}_{\omega}(\cdot) F(\cdot, \lambda, v)\right\|_{L^{\infty}([0,1])}\right) .
$$

By Lemma 2.7,

$$
\begin{aligned}
\left|\mathcal{E}_{\omega\left(\zeta_{n}\right)}(x) F\left(x, \zeta_{n}, v\right)\right| \leq & \frac{1}{\left|\zeta_{n}\right|} e^{\left|\operatorname{Im} \omega\left(\zeta_{n}\right)\right| x} e^{2\|q\|_{1}} \\
& +\max _{ \pm}\left|\int_{0}^{x}\left(P p(s)+q_{x}(s)\right) e^{ \pm \mathrm{i} \omega\left(\zeta_{n}\right)(x-2 s)} \mathrm{d} s\right|,
\end{aligned}
$$

and by Lemma D.2 in [3],

$$
\sum_{n \in \mathbb{Z}} \max _{ \pm}\left|\int_{0}^{x}\left(P p(s)+q_{x}(s)\right) e^{ \pm \mathrm{i} \omega\left(\zeta_{n}\right)(x-2 s)} \mathrm{d} s\right|^{2} \leq 2 e^{2 b}\|v\|_{1}, \quad 0 \leq x \leq 1,
$$

where $b=\sup _{n \in \mathbb{Z}}\left|\omega\left(\zeta_{n}\right)-n \pi\right|$. Altogether, we thus have proved $\sum_{n \in \mathbb{Z}}\left|\mathcal{M}\left(x, \zeta_{n}, v\right)-\mathcal{E}_{\omega\left(\zeta_{n}\right)}(x)\right|^{2}<\infty$. In view of Lemma 2.8, the latter estimate holds uniformly on bounded subsets of $H_{c}^{1}$ and on subsets of sequences $\left(\zeta_{n}\right)_{n}$ in $\mathbb{C}^{*}$ so that $\left(\omega\left(\zeta_{n}\right)-n \pi\right)_{n}$ is bounded in $\ell_{\mathbb{C}}^{\infty}$. To obtain the claimed estimate for $\dot{\mathcal{M}}\left(x, \zeta_{n}, v\right)-\dot{\mathcal{E}}_{\omega\left(\zeta_{n}\right)}(x)$ we apply Cauchy's estimate to $\lambda$-discs $D_{\zeta_{n}}$ of fixed radius around each $\zeta_{n}$ to get

$$
\left|\dot{\mathcal{M}}\left(x, \zeta_{n}, v\right)-\dot{\mathcal{E}}_{\omega\left(\zeta_{n}\right)}(x)\right| \leq O\left(\sup _{\lambda \in D_{\zeta_{n}}}\left|\mathcal{M}(x, \lambda, v)-\mathcal{E}_{\omega(\lambda)}(x)\right|\right) .
$$


Since the radii of the discs $D_{\zeta_{n}}$ do not depend on $n$ one then concludes that

$$
\sup _{0 \leq x \leq 1} \sum_{n \in \mathbb{Z}}\left|\dot{\mathcal{M}}\left(x, \zeta_{n}, v\right)-\dot{\mathcal{E}}_{\omega\left(\zeta_{n}\right)}(x)\right|^{2}<\infty
$$

where the estimate holds uniformly in the claimed sense. Altogether this establishes the first claim.

(ii) For sequences $\left(\zeta_{n}\right)_{n}$ with the stronger asymptotics $\omega\left(\zeta_{n}\right)=n \pi+\ell_{n}^{2}$, we have

$$
\mathcal{E}_{\omega\left(\zeta_{n}\right)}(x)=e^{-R \omega\left(\zeta_{n}\right) x}=e^{-R n \pi x}+\ell_{n}^{2}=\mathcal{E}_{\omega(n \pi)}(x)+\ell_{n}^{2}
$$

implying the claimed estimate.

Theorem 2.11. Uniformly on bounded subsets of $H_{c}^{2}$ and on subsets of biinfinite sequences of complex numbers with $\zeta_{n}=n \pi+O(1),\left|\zeta_{n}\right|>\frac{1}{4}$,

$$
\begin{aligned}
& \sup _{0 \leq x \leq 1}\left|\mathcal{M}\left(x, \zeta_{n}, v\right)-\mathcal{E}_{\omega\left(\zeta_{n}\right)}(x)\right|=O(1 / n), \\
& \sup _{0 \leq x \leq 1}\left|\dot{\mathcal{M}}\left(x, \zeta_{n}, v\right)-\dot{\mathcal{E}}_{\omega\left(\zeta_{n}\right)}(x)\right|=O(1 / n) .
\end{aligned}
$$

If in addition $\zeta_{n}=n \pi+\ell_{n}^{2}$, then

$$
\begin{aligned}
& \sup _{0 \leq x \leq 1}\left|\mathcal{M}\left(x, \zeta_{n}, v\right)-\mathcal{E}_{n \pi}(x)\right|=\ell_{n}^{2}, \\
& \sup _{0 \leq x \leq 1}\left|\dot{\mathcal{M}}\left(x, \zeta_{n}, v\right)-\dot{\mathcal{E}}_{n \pi}(x)\right|=\ell_{n}^{2} .
\end{aligned}
$$

Note that $\dot{\mathcal{E}}_{\omega\left(\zeta_{n}\right)}=-x \dot{\omega}\left(\zeta_{n}\right) R e^{-R \omega\left(\zeta_{n}\right) x}=-x R \mathcal{E}_{\omega\left(\zeta_{n}\right)}(x)+O\left(\frac{1}{n}\right)$.

Proof. The first estimate is a consequence of Theorem 2.9 and the second one is then obtained by applying Cauchy's estimate. If the sequence $\zeta_{n}$ satisfies in addition $\zeta_{n}=n \pi+\ell_{n}^{2}$ then $\mathcal{E}_{\omega\left(\zeta_{n}\right)}(x)=\mathcal{E}_{n \pi}(x)+\ell_{n}^{2}$.

For later reference we state the asymptotics of $M=T^{-1} \mathcal{M} T$, corresponding to the ones obtained for $\mathcal{M}$. Introduce

$$
E_{\omega(\lambda)}(x):=M(x, \lambda, 0)=T^{-1} \mathcal{E}_{\omega(\lambda)}(x) T=\left(\begin{array}{cc}
\cos (\omega(\lambda) x) & \sin (\omega(\lambda) x) \\
-\sin (\omega(\lambda) x) & \cos (\omega(\lambda) x)
\end{array}\right) .
$$

Since

$$
\begin{aligned}
\dot{E}_{\omega(\lambda)}(x) & =-T^{-1} x \dot{\omega}(\lambda) R e^{-R \omega(\lambda) x} T=-x \dot{\omega}(\lambda) T^{-1} R T E_{\omega(\lambda)}(x) \\
& =x J E_{\omega(\lambda)}(x)+O\left(e^{|\operatorname{Im} \omega(\lambda)| x} /|\lambda|^{2}\right) .
\end{aligned}
$$

Theorems 2.9-2.11 then yield the following results.

Theorem 2.12. $M$ and $\dot{M}$ have the following asymptotics: 
(i) For $|\lambda| \rightarrow \infty$ locally uniformly on $[0,1] \times H_{c}^{1}$,

$$
\begin{aligned}
& M(x, \lambda, v)=E_{\omega(\lambda)}(x)+o\left(e^{|\operatorname{Im} \omega(\lambda)| x}\right), \\
& \dot{M}(x, \lambda, v)=x J E_{\omega(\lambda)}(x)+o\left(e^{|\operatorname{Im} \omega(\lambda)| x}\right) .
\end{aligned}
$$

(ii) For $|\lambda| \rightarrow \infty$ uniformly on bounded subsets of $[0,1] \times H_{c}^{2}$,

$$
\begin{aligned}
& M(x, \lambda, v)=E_{\omega(\lambda)}(x)+O\left(e^{|\operatorname{Im} \omega(\lambda)| x} /|\omega(\lambda)|\right), \\
& \dot{M}(x, \lambda, v)=x J E_{\omega(\lambda)}(x)+O\left(e^{|\operatorname{Im} \omega(\lambda)| x} /|\omega(\lambda)|\right) .
\end{aligned}
$$

Furthermore for bi-infinite sequences of complex numbers $\left(\zeta_{n}\right)_{n} \subset \mathbb{C}^{*}$ with $\left|\zeta_{n}\right| \geq$ $\frac{1}{4}$ one has:

(iii) If $\zeta_{n}=n \pi+O(1)$, then

$$
M\left(x, \zeta_{n}, v\right)=E_{\omega\left(\zeta_{n}\right)}(x)+\ell_{n}^{2} \text { and } \dot{M}\left(x, \zeta_{n}, v\right)=x J E_{\omega\left(\zeta_{n}\right)}(x)+\ell_{n}^{2},
$$

uniformly on $[0,1]$, on bounded subsets of $H_{c}^{2}$, and on subsets of sequences $\left(\zeta_{n}\right)_{n}$ where $\left(\omega\left(\zeta_{n}\right)-n \pi\right)_{n}$ is bounded in $\ell_{\mathbb{C}}^{\infty}$.

(iv) If $\zeta_{n}=n \pi+\ell_{n}^{2}$, then

$$
M\left(x, \zeta_{n}, v\right)=E_{n \pi}(x)+\ell_{n}^{2} \text { and } \dot{M}\left(x, \zeta_{n}, v\right)=x J E_{n \pi}(x)+\ell_{n}^{2},
$$

uniformly on $[0,1]$, on bounded subsets of $H_{c}^{1}$, and on subsets of sequences $\left(\zeta_{n}\right)_{n}$ where $\left(\omega\left(\zeta_{n}\right)-n \pi\right)_{n}$ is bounded in $\ell_{\mathbb{C}}^{2}$.

(v) If $\zeta_{n}=n \pi+O(1)$ and $v \in H_{c}^{2}$, then

$M\left(x, \zeta_{n}, v\right)=E_{\omega\left(\zeta_{n}\right)}(x)+O(1 / n)$ and $\dot{M}\left(x, \zeta_{n}, v\right)=x J E_{\omega\left(\zeta_{n}\right)}(x)+O(1 / n)$

uniformly on $[0,1]$, on bounded subsets of $H_{c}^{2}$, and on subsets of sequences $\zeta_{n}$ where $\left(\zeta_{n}-n \pi\right)_{n}$ is bounded in $\ell_{\mathbb{C}}^{\infty}$.

Recall that by $(2.29), M^{-1}=\left(\begin{array}{cc}m_{4} & -m_{2} \\ -m_{3} & m_{1}\end{array}\right)$. Furthermore, $E_{\omega(\lambda)}^{-1}=E_{-\omega(\lambda)}=$ $E_{\omega(-\lambda)}$ and hence

$$
\left(E_{\omega(\lambda)}^{-1}\right)^{\cdot}=-\dot{E}_{\omega(-\lambda)}(x)=-x J E_{\omega(-\lambda)}(x)+O\left(e^{|\operatorname{Im} \omega(-\lambda)| x} / \lambda^{2}\right) .
$$

Theorem 2.12 then leads to the following results for $M^{-1}$.

Corollary 2.13. $M^{-1}$ and $\left(M^{-1}\right)^{\cdot}$ satisfy the following estimates:

(i) For $|\lambda| \rightarrow \infty$ locally uniformly on $[0,1] \times H_{c}^{1}$,

$$
\begin{aligned}
M^{-1}(x, \lambda, v) & =E_{\omega(-\lambda)}(x)+o\left(e^{|\operatorname{Im} \omega(\lambda)| x}\right), \\
\left(M^{-1}\right)^{\cdot}(x, \lambda, v) & =-x J E_{\omega(-\lambda)}(x)+o\left(e^{|\operatorname{Im} \omega(\lambda)| x}\right) .
\end{aligned}
$$


(ii) $\operatorname{For}|\lambda| \rightarrow \infty$,

$$
M^{-1}(x, \lambda, v)=E_{\omega(-\lambda)}(x)+o\left(e^{|\operatorname{Im} \omega(\lambda)| x} /|\omega(\lambda)|\right)
$$

uniformly on bounded subsets of $[0,1] \times H_{c}^{2}$, and

$$
\left(M^{-1}\right)^{\cdot}(x, \lambda, v)=-x J E_{\omega(-\lambda)}(x)+o\left(e^{|\operatorname{Im} \omega(\lambda)| x} /|\omega(\lambda)|\right) .
$$

Furthermore for bi-infinite sequences of complex numbers $\left(\zeta_{n}\right)_{n}$ in $\mathbb{C}^{*}$ with $\left|\zeta_{n}\right| \geq \frac{1}{4}$ one has:

(iii) If $\zeta_{n}=n \pi+O(1)$, then

$M^{-1}\left(x, \zeta_{n}, v\right)=E_{\omega\left(-\zeta_{n}\right)}(x)+\ell_{n}^{2}$ and $\left(M^{-1}\right)^{\cdot}\left(x, \zeta_{n}, v\right)=-x J E_{\omega\left(-\zeta_{n}\right)}(x)+\ell_{n}^{2}$ uniformly on $[0,1]$, on bounded subsets of $H_{c}^{1}$, and on subsets of sequences $\left(\zeta_{n}\right)_{n}$, where $\left(\omega\left(\zeta_{n}\right)-n \pi\right)_{n}$ is bounded in $\ell_{\mathbb{C}}^{\infty}$.

(iv) If $\zeta_{n}=n \pi+\ell_{n}^{2}$, then

$$
M^{-1}\left(x, \zeta_{n}, v\right)=E_{-n \pi}(x)+\ell_{n}^{2} \text { and }\left(M^{-1}\right)^{\cdot}\left(x, \zeta_{n}, v\right)=-x J E_{-n \pi}(x)+\ell_{n}^{2}
$$

uniformly on $[0,1]$, on bounded subsets of $H_{c}^{1}$, and on subsets of sequences $\left(\zeta_{n}\right)_{n}$, where $\left(\omega\left(\zeta_{n}\right)-n \pi\right)_{n}$ is bounded in $\ell_{\mathbb{C}}^{2}$.

(v) If $\zeta_{n}=n \pi+O(1)$ and $v \in H_{c}^{2}$, then

$$
M^{-1}\left(x, \zeta_{n}, v\right)=E_{\omega\left(-\zeta_{n}\right)}(x)+O(1 / n)
$$

and

$$
\left(M^{-1}\right)^{\cdot}\left(x, \zeta_{n}, v\right)=-x J E_{\omega\left(-\zeta_{n}\right)}(x)+O(1 / n)
$$

uniformly on $[0,1]$, on bounded subsets of $H_{c}^{2}$, and sequences $\left(\zeta_{n}\right)_{n}$, where $\left(\zeta_{n}-n \pi\right)_{n}$ is bounded in $\ell_{\mathbb{C}}^{\infty}$.

In order to study the periodic spectrum of the operator $Q$, its discriminant plays an important role. For any $v \in H_{c}^{1}$, let

$$
\grave{M}(\lambda, v):=\left.M(x, \lambda, v)\right|_{x=1} \text { and } \grave{M}(\lambda, v)=:\left(\begin{array}{cc}
\grave{m}_{1} & \grave{m}_{2} \\
\grave{m}_{3} & \grave{m}_{4}
\end{array}\right)
$$

as well as $\grave{\mathcal{M}}(\lambda, v):=\mathcal{M}(1, \lambda, v)$. The discriminant and anti-discriminant are then defined as follows:

$$
\Delta(\lambda, v):=\frac{1}{2} \operatorname{tr} \grave{M}(\lambda, v)=\frac{1}{2} \operatorname{tr} \grave{\mathcal{M}}(\lambda, v), \delta(\lambda, v):=\left(\grave{m}_{1}(\lambda, v)-\grave{m}_{4}(\lambda, v)\right) / 2 .
$$

Lemma 2.14. $\Delta$ and $\delta$ are analytic maps on $\mathbb{C}^{*} \times H_{c}^{1}$ and have the following symmetries: for any $\lambda \in \mathbb{C}^{*}$ and $v \in H_{c}^{1}$, 
(i) (Reflection in $\lambda$ )

$$
\Delta(-\lambda, v)=\Delta(\lambda, v), \quad \delta(-\lambda, v)=\delta(\lambda, v) .
$$

(ii) (Reciprocity in $\lambda$ )

$$
\Delta\left(\frac{1}{16 \lambda}, q, p\right)=\Delta(\lambda,-q, p), \quad \delta\left(\frac{1}{16 \lambda}, q, p\right)=\delta(\lambda,-q, p) .
$$

(iii) (Conjugation)

$$
\Delta(\bar{\lambda}, \bar{v})=\overline{\Delta(\lambda, v)}, \quad \delta(\bar{\lambda}, \bar{v})=\overline{\delta(\lambda, v)} .
$$

(iv) (Reflection of $v$ )

$$
\Delta(\lambda,-v)=\Delta(\lambda, v), \quad \delta(\lambda,-v)=-\delta(\lambda, v) .
$$

(v) (Real potentials) If the components $q$ and $p$ of $v$ are real valued then $\Delta(\lambda, v)$ and $\delta(\lambda, v)$ are real for any $\lambda \in \mathbb{R} \cup \mathrm{i} \mathbb{R}$.

(vi) (Purely imaginary potentials) If $q$ and $p$ take values in $i \mathbb{R}$ then $\Delta(\lambda, v)$ is real and $\delta(\lambda, v)$ is purely imaginary for any $\lambda \in \mathbb{R} \cup \mathrm{i} \mathbb{R}$.

Proof. Items (i)-(iv) follow from Proposition 2.1.

(v) By (i) and (iii) one has for $v$ real that for any $\lambda \in \mathbb{R} \cup \mathrm{i} \mathbb{R}$

$$
\overline{\Delta(\lambda, v)}=\overline{\Delta(\bar{\lambda}, \bar{v})}=\Delta(\lambda, v) \quad \text { and } \quad \overline{\delta(\lambda, v)}=\overline{\delta(\bar{\lambda}, \bar{v})}=\delta(\lambda, v) .
$$

(vi) In case $v$ is purely imaginary it follows from (i), (iii), and (iv) that for any $\lambda \in \mathbb{R} \cup \mathrm{i} \mathbb{R}$

$$
\overline{\Delta(\lambda, v)}=\overline{\Delta(\bar{\lambda},-\bar{v})}=\Delta(\lambda, v) \quad \text { and } \quad \overline{\delta(\lambda, v)}=\overline{\delta(\bar{\lambda},-\bar{v})}=-\delta(\lambda, v) .
$$

The latter lemma leads to the following

Corollary 2.15. Discriminant and anti-discriminant together with their $\lambda$ derivatives are real analytic on $\mathbb{C}^{*} \times H_{c}^{1}$. On any closed, bounded subset of $\mathbb{C}^{*} \times$ $H_{c}^{1}, \Delta$ and $\delta$ are compact and bounded. More precisely, for any compact subset $K \subset \mathbb{C}^{*}$ and any closed, bounded subset $V \subset H_{c}^{1}$, the map $V \rightarrow L_{\mathbb{C}}^{\infty}(K), v \mapsto$ $(\lambda \mapsto \Delta(\lambda, v))$ is compact in the sense of Definition 2.4.

For later reference we record the following formulas for $\Delta(\lambda, v)$ and $\delta(\lambda, v)$ at the zero potential $v=0$. Recall that $\omega(\lambda)=\lambda-\frac{1}{16 \lambda}$. Taking into account that by $(2.33), \grave{M}(\lambda, 0)=E_{\omega(\lambda)}(1)$, the following holds.

Lemma 2.16. For any $\lambda \in \mathbb{C}^{*}$

$$
\Delta(\lambda, 0)=\cos (\omega(\lambda)), \dot{\Delta}(\lambda, 0)=-\left(1+\frac{1}{16 \lambda^{2}}\right) \sin (\omega(\lambda)), \delta(\lambda, 0)=\dot{\delta}(\lambda, 0)=0
$$

As a consequence $\Delta^{2}(\lambda, 0)-1=-\sin ^{2}(\omega(\lambda))$. 
To obtain rough asymptotics of the periodic eigenvalues we need to compare $\Delta(\lambda, v)$ with $\Delta(\lambda, 0)$. Recall that the domains $D_{n}, n \in \mathbb{Z}$, were introduced in the introduction (cf. also (3.1) below).

Lemma 2.17. For any given $v \in H_{c}^{1}$, the following asymptotics for $|\lambda| \rightarrow \infty$ hold on $\mathbb{C} \backslash \bigcup_{n \geq 1} D_{n} \cup\left(-D_{n}\right)$

$$
\begin{aligned}
\Delta^{2}(\lambda)-1 & =-\sin ^{2}(\omega(\lambda))(1+o(1))=-\sin ^{2}(\lambda)(1+o(1)), \\
\dot{\Delta}(\lambda) & =-\sin (\lambda)(1+o(1)) .
\end{aligned}
$$

These estimates hold locally uniformly on $H_{c}^{1}$.

Proof. By Theorem 2.12 (ii), $\Delta(\lambda, v)=\cos (\omega(\lambda))+o\left(e^{|\operatorname{Im} \omega(\lambda)|}\right)$, and thus

$$
\begin{aligned}
\Delta^{2}(\lambda, v) & -1 \\
& =-\sin ^{2}(\omega(\lambda))\left(1+\frac{o\left(e^{|\operatorname{Im} \omega(\lambda)|}\right) \cos (\omega(\lambda))}{\sin ^{2}(\omega(\lambda))}+\frac{o\left(e^{2|\operatorname{Im} \omega(\lambda)|}\right)}{\sin ^{2}(\omega(\lambda))}\right) .
\end{aligned}
$$

For $\lambda \in \mathbb{C}^{*} \backslash \bigcup_{n \geq 1} D_{n} \cup\left(-D_{n}\right)$ there exists $m \in \mathbb{Z}$ with $m \pi+\pi / 3 \leq \operatorname{Re} \lambda \leq(m+$ 1) $\pi-\pi / 3$. If in addition, $\lambda$ is sufficiently large, then $\omega(\lambda)=\lambda-\frac{1}{16 \lambda}$ satisfies $m \pi+\pi / 4 \leq \operatorname{Re} \omega(\lambda) \leq(m+1) \pi-\pi / 4$. Hence $|\sin (\operatorname{Re} \omega(\lambda))| \geq \frac{1}{\sqrt{2}}$ and

$$
\begin{aligned}
|\sin (\omega(\lambda))| & =\mid \sin (\operatorname{Re} \omega(\lambda)) \cos (\mathrm{i} \operatorname{Im} \omega(\lambda))+\cos (\operatorname{Re} \omega(\lambda) \sin (\mathrm{i} \operatorname{Im} \omega(\lambda)) \mid \\
& \geq \cosh (|\operatorname{Im} \omega(\lambda)|) / \sqrt{2} \geq e^{|\operatorname{Im} \omega(\lambda)|} / \sqrt{2} .
\end{aligned}
$$

It follows that for $\lambda \in \mathbb{C}^{*} \backslash \bigcup_{n \geq 1} D_{n} \cup\left(-D_{n}\right)$ sufficiently large

$$
\left|\frac{\cos (\omega(\lambda))}{\sin (\omega(\lambda))}\right| \leq \frac{e^{|\operatorname{Im} \omega(\lambda)|}}{|\sin (\omega(\lambda))|} \leq \sqrt{2},
$$

and hence the expression inside the large parentheses of (2.39) is $1+o(1)$. The asymptotics (2.37) then follow since

$$
\begin{aligned}
\sin (\omega(\lambda)) & =\sin (\lambda) \cos \left(\frac{1}{16 \lambda}\right)-\sin \left(\frac{1}{16 \lambda}\right) \cos (\lambda) \\
& =\sin (\lambda)\left(1+O\left(\frac{1}{\lambda^{2}}\right)+O\left(\frac{1}{\lambda}\right) \frac{\cos (\lambda)}{\sin (\lambda)}\right)
\end{aligned}
$$

and

$$
\left|\frac{\cos (\lambda)}{\sin (\lambda)}\right| \leq \frac{e^{|\operatorname{Im} \lambda|}}{\frac{1}{\sqrt{2}} e^{|\operatorname{Im} \lambda|}} \leq \sqrt{2} .
$$

Concerning (2.38), note that by Theorem 2.12 (i),

$$
\dot{M}(x, \lambda, v)=x J E_{\omega(\lambda)}(x)+o\left(e^{|\operatorname{Im} \omega(\lambda)| x}\right)
$$

implying that

$$
\dot{\Delta}(\lambda, v)=-\sin (\omega(\lambda))+o\left(e^{|\operatorname{Im} \omega(\lambda)|}\right) .
$$

A similar argument as the one above then yields the claimed asymptotics. 


\section{Spectra}

The main purpose of this section is to study the asymptotics of the periodic, the Dirichlet, and the Neumann eigenvalues of the operator $Q(v)$ for $v \in H_{c}^{1}$. They provide the setup for the proofs of Theorem 1.1 and Theorem 1.2 in Section 4. Recall that in Section 1, we introduced the domains $D_{0}:=$ $\left\{z \in \mathbb{C}:\left|z-\frac{1}{4}\right|<\frac{1}{4 \pi}\right\}$ and for any $n \geq 1$,

$$
D_{n}:=\{\lambda \in \mathbb{C}:|\lambda-n \pi|<\pi / 3\}, \quad D_{-n}:=\left\{\lambda \in \mathbb{C}: \frac{1}{16 \lambda} \in D_{n}\right\}
$$

as well as $B_{0}:=\{\lambda \in \mathbb{C}:|\lambda| \leq \pi / 2\}$ and for any $n \geq 1$ and

$$
\begin{aligned}
B_{n} & :=\{\lambda \in \mathbb{C}:|\lambda|<n \pi+\pi / 2\}, \\
B_{-n} & :=\left\{\lambda \in \mathbb{C}:|\lambda| \leq \frac{1}{16(n \pi+\pi / 2)}\right\},
\end{aligned}
$$

and we denote by $A_{n}$ the open annulus

$$
A_{n}:=B_{n} \backslash B_{-n}
$$

Furthermore, by the definition (2.35)

$$
\grave{M}(\lambda, v)=\left.M(x, \lambda, v)\right|_{x=1} \quad \text { and } \quad \grave{M}(\lambda, v)=\left(\begin{array}{ll}
\grave{m}_{1} & \grave{m}_{2} \\
\grave{m}_{3} & \grave{m}_{4}
\end{array}\right)
$$

as well as $\grave{\mathcal{M}}(\lambda, v)=\left.\mathcal{M}(x, \lambda, v)\right|_{x=1}$. Denote by $Q_{\text {dir }}$ the operator $Q=Q_{1} \partial_{x}+$ $Q_{0}$ with domain

$$
H_{\mathrm{dir}}:=\left\{F=\left(F_{1}, F_{2}, F_{3}, F_{4}\right) \in H^{1}\left([0,1], \mathbb{C}^{4}\right): F_{1}(0)=F_{1}(1)=0\right\} .
$$

Its spectrum is discrete and coincides with the Dirichlet spectrum of the spectral problem (2.2), defined as the set of eigenvalues with eigenfunctions $f=\left(f_{1}, f_{2}\right) \in$ $H^{1}\left([0,1], \mathbb{C}^{2}\right)$ such that $f_{1}(0)=0=f_{1}(1)$. Clearly, for any $v \in H_{c}^{1}, \mu \in \mathbb{C}^{*}$ is a Dirichlet eigenvalue of $(2.2)$ if there exists $a \in \mathbb{C}^{*}$ such that

$$
\grave{M}(\mu, v)\left(\begin{array}{l}
0 \\
1
\end{array}\right)=a\left(\begin{array}{l}
0 \\
1
\end{array}\right) .
$$

We thus have the following

Theorem 3.1. The Dirichlet spectrum of $Q(v)$ with $v \in H_{c}^{1}$ is the zero set of the function $\chi_{D}(\lambda):=\grave{m}_{2}(\lambda),\left\{\mu \in \mathbb{C}^{*}: \chi_{D}(\mu)=0\right\}$. Furthermore, the multiplicity $\operatorname{Mult}\left(\mu, \chi_{D}\right)$ of a root $\mu$ of $\grave{m}_{2}$ is equal to the algebraic multiplicity $\operatorname{Mult}_{a}(\mu)$ of $\mu$ as a Dirichlet eigenvalue, defined as the dimension of the (finite dimensional) vector space $\bigcup_{n \geq 1} \operatorname{ker}\left(\mu-Q_{\text {dir }}(v)\right)^{n}$. The function $\chi_{D}$ is an analytic and compact function on $\mathbb{C}^{*} \times H_{c}^{1}$. For $v=0, \chi_{D}(\lambda, 0)=\sin (\omega(\lambda))$. 
All the assertions of Theorem 3.1 are shown in a straightforward way except the one on the multiplicity of the roots of $\chi_{D}$, which we state separately in Lemma 3.5 below. To prove it, we first need to discuss some elementary properties of the Dirichlet eigenvalues and $\chi_{D}$.

Lemma 3.2. For any $(\lambda, v) \in \mathbb{C}^{*} \times H_{c}^{1}$

(i) $\chi_{D}(-\lambda, v)=-\chi_{D}(\lambda, v)$ and $\chi_{D}\left(\frac{1}{16 \lambda}, q, p\right)=-e^{-q(0)} \chi_{D}(\lambda,-q, p)$.

(ii) $\chi_{D}(\bar{\lambda}, \bar{v})=\overline{\chi_{D}(\lambda, v)}$ and $\chi_{D}(\lambda,-v)=-\grave{m}_{3}(\lambda, v)$.

(iii) For $|\lambda| \rightarrow \infty$ with $\lambda \notin \bigcup_{n \geq 1} D_{n} \cup\left(-D_{n}\right), \chi_{D}(\lambda, v)=\chi_{D}(\lambda, 0)(1+o(1))$ locally uniformly in $v \in H_{c}^{1}$.

Proof. (i) Using that

$$
\left(\begin{array}{c}
\chi_{D}(\lambda, v) \\
0
\end{array}\right)=\left(\begin{array}{c}
\grave{m}_{2}(\lambda, v) \\
0
\end{array}\right)=\left(\begin{array}{ll}
1 & 0 \\
0 & 0
\end{array}\right) \grave{M}(\lambda, v)\left(\begin{array}{l}
0 \\
1
\end{array}\right)
$$

we obtain by Proposition 2.1

$$
\begin{aligned}
\left(\begin{array}{c}
\chi_{D}(-\lambda, v) \\
0
\end{array}\right) & =\left(\begin{array}{ll}
1 & 0 \\
0 & 0
\end{array}\right) \grave{M}(-\lambda, v)\left(\begin{array}{l}
0 \\
1
\end{array}\right) \\
& =-\left(\begin{array}{ll}
1 & 0 \\
0 & 0
\end{array}\right) R \grave{M}(\lambda, v) R\left(\begin{array}{l}
0 \\
1
\end{array}\right)=\left(\begin{array}{c}
-\grave{m}_{2}(\lambda, v) \\
0
\end{array}\right), \\
\left(\begin{array}{c}
\chi_{D}\left(\frac{1}{16 \lambda}, v\right) \\
0
\end{array}\right) & =\left(\begin{array}{ll}
1 & 0 \\
0 & 0
\end{array}\right) \grave{M}\left(\frac{1}{16 \lambda}, q, p\right)\left(\begin{array}{l}
0 \\
1
\end{array}\right) \\
& =-\left(\begin{array}{ll}
1 & 0 \\
0 & 0
\end{array}\right) R e^{\mathrm{i} R q(0) / 2} \grave{M}(\lambda,-q, p) e^{-\mathrm{i} R q(0) / 2} R\left(\begin{array}{l}
0 \\
1
\end{array}\right) \\
& =-\left(\begin{array}{cc}
\mathrm{i} e^{-q(0) / 2} & 0 \\
0 & 0
\end{array}\right) \grave{M}(\lambda,-q, p)\left(\begin{array}{c}
0 \\
-\mathrm{i} e^{-q(0) / 2}
\end{array}\right) \\
& =-\left(\begin{array}{c}
e^{-q(0)} \grave{m}_{2}(\lambda,-q, p) \\
0
\end{array}\right) .
\end{aligned}
$$

(ii) is proved in a similar way as item (i).

(iii) By the same argument as in the proof of Lemma 2.17 one obtains the claimed asymptotics $\chi_{D}(\lambda, v)=\chi_{D}(\lambda, 0)(1+o(1))$.

As usual we denote by $\sqrt[+]{\lambda}$ the principal branch of the square root defined for $\lambda$ in $\mathbb{C} \backslash(-\infty, 0]$ and determined by $\sqrt[+]{1}=1$.

Lemma 3.3. The Dirichlet eigenvalues at $v=0$ are $\frac{1}{4},-\frac{1}{4}$ and

$$
\frac{n \pi}{2}\left(\sqrt[+]{1+\frac{1}{4 n^{2} \pi^{2}}}+1\right), \quad \frac{n \pi}{2}\left(\sqrt[+]{1+\frac{1}{4 n^{2} \pi^{2}}}-1\right), \quad n \neq 0
$$

each eigenvalue having multiplicity one. 
A rough localization of the Dirichlet eigenvalues is provided by the following

Lemma 3.4 (Counting Lemma). For each potential in $H_{c}^{1}$, there exist a neighborhood $U$ in $H_{c}^{1}$ and an integer $N>0$ so that for any $v \in U$, the function $\lambda \mapsto \chi_{D}(\lambda, v)$ has exactly one root in each of the domains $D_{n},-D_{n}, D_{-n},-D_{-n}$ for any $n>N$ and exactly $2+4 N$ in the annulus $A_{N}$, counted with their multiplicities. There are no other roots.

Proof. By Lemma 3.2, for $|\lambda| \rightarrow \infty$ with $\lambda \notin \bigcup_{n \geq 1} D_{n} \cup\left(-D_{n}\right), \chi_{D}(\lambda, v)=$ $\chi_{D}(\lambda, 0)(1+o(1))$ locally uniformly in $v$. Hence, for any potential in $H_{c}^{1}$ there is a neighborhood $U$ and an integer $N \geq 1$ such that for any $v \in U$

$$
\begin{aligned}
\left|\chi_{D}(\lambda, v)-\chi_{D}(\lambda, 0)\right| & <\left|\chi_{D}(\lambda, 0)\right|, \\
\left|\chi_{D}(\lambda,(-q, p))-\chi_{D}(\lambda, 0)\right| & <\left|\chi_{D}(\lambda, 0)\right|
\end{aligned}
$$

on the boundaries of the discs $D_{n},-D_{n}$, and $B_{n}$ for any $n \geq N$. It follows by Rouché's theorem that $\chi_{D}(\cdot, v)$ has as many roots inside any of the discs $\pm D_{n}$, $n \geq N$, as $\chi_{D}(\cdot, 0)$. There are no other roots in $\mathbb{C}^{*} \backslash\left(B_{N} \bigcup_{n \geq N}\left(D_{n} \cup-D_{n}\right)\right)$. By Lemma 3.2 (i)

$$
\begin{aligned}
\left|e^{q(0)} \chi_{D}\left(\frac{1}{16 \lambda},(q, p)\right)-\chi_{D}\left(\frac{1}{16 \lambda}, 0\right)\right| & =\left|\chi_{D}(\lambda,(-q, p))-\chi_{D}(\lambda, 0)\right| \\
& <\left|\chi_{D}(\lambda, 0)\right| .
\end{aligned}
$$

Since $\chi_{D}(\cdot, v)$ has the same roots as $e^{q(0)} \chi_{D}(\cdot, v)$ and $\chi_{D}\left(\frac{1}{16 \lambda}, 0\right)=\chi_{D}(\lambda, 0)$ it follows that $\chi_{D}(\lambda, v)$ has as many roots as $\chi_{D}(\lambda, 0)$ inside any of the discs $D_{-n},-D_{-n}$ with $n>N$. It remains to count the roots inside $A_{n}$ with $n \geq N$. In order to apply Rouché's theorem we need to estimate $\chi_{D}$ on the boundary of $B_{-n}$. Arguing as above one concludes that for any $\lambda$ on the boundary of $B_{n}$ with $n \geq N$ and $t, t+s \in[0,1]$,

$$
\begin{aligned}
\mid e^{(t+s) q(0)} \chi_{D}\left(\frac{1}{16 \lambda},(t+s) v\right) & -e^{t q(0)} \chi_{D}\left(\frac{1}{16 \lambda}, t v\right) \mid \\
& =\left|\chi_{D}(\lambda,-(t+s) v)-\chi_{D}(\lambda,-t v)\right| \\
& <\frac{1}{2}\left|\chi_{D}(\lambda,-t v)\right|=\frac{1}{2}\left|e^{t q(0)} \chi_{D}\left(\frac{1}{16 \lambda}, t v\right)\right| .
\end{aligned}
$$

After division by $\left|e^{t q(0)}\right|$ one gets

$$
\left|e^{s q(0)} \chi_{D}\left(\frac{1}{16 \lambda},(t+s) v\right)-\chi_{D}\left(\frac{1}{16 \lambda}, t v\right)\right| \leq \frac{1}{2}\left|\chi_{D}\left(\frac{1}{16 \lambda}, t v\right)\right| .
$$

Choose $\epsilon>0$ such that

$$
\left|e^{s q(0)}-1\right|\left|\chi_{D}\left(\frac{1}{16 \lambda},(t+s) v\right)\right|<\frac{1}{2}\left|\chi_{D}\left(\frac{1}{16 \lambda}, t v\right)\right|
$$


for $\lambda$ on the boundary of $B_{n}$ with $n \geq N, t \in[0,1]$, and $0 \leq s<\epsilon$. Then

$$
\left|\chi_{D}(\lambda,(t+s) v)-\chi_{D}(\lambda, t v)\right|<\left|\chi_{D}(\lambda, t v)\right|
$$

on the boundary of $A_{n}$. By Rouché's Theorem it then follows that the number of roots of $\chi_{D}(\cdot, t v)$ inside $A_{n}$ is independent of $t \in[0,1]$. Since $\chi_{D}(\cdot, 0)$ has $2+$ $4 N$ roots inside $A_{N}$ so does $\chi_{D}(\cdot, v)$. Since $\left(A_{n}\right)_{n \geq N}$ is a covering of $\mathbb{C}^{*}$, there are no roots in $\mathbb{C}^{*} \backslash\left(A_{N} \cup \bigcup_{n \geq N} D_{n} \cup\left(-D_{n}\right)\right)$.

Since by Lemma 3.2 (i), $\chi_{D}(-\lambda, v)=-\chi_{D}(\lambda, v)$, it is enough to consider the Dirichlet eigenvalues of $Q(v)$ in

$$
\mathbb{C}^{+}:=\{\lambda \in \mathbb{C}: \operatorname{Re} \lambda>0\} \cup \mathrm{i} \mathbb{R}_{>0} .
$$

For any $v \in H_{c}^{1}$, these eigenvalues, when counted with their multiplicities $\operatorname{Mult}_{a}(\mu)$, can be listed as a bi-infinite sequence

$$
0 \preceq \cdots \preceq \mu_{-2} \preceq \mu_{-1} \preceq \mu_{0} \preceq \mu_{1} \preceq \mu_{2} \preceq \cdots .
$$

Here $\preceq$ is the ordering of complex numbers in $\mathbb{C}^{+}$defined as follows: for $a, b \in$ $\mathbb{C}^{+}, a \preceq b$,

$$
[|a|<|b|] \text { or }[|a|=|b| \text { and } \operatorname{Im} a \leq \operatorname{Im} b] .
$$

Note that $\preceq$ is a total ordering of $\mathbb{C}^{+}$. One of its feature is that for any $a \in \mathbb{C}^{+}$, $a \preceq \mathrm{i}|a|$. In particular, ordering the Dirichlet eigenvalues in this way one has that $\mu_{n}=n \pi+o(1)$ and $\frac{1}{16 \mu_{-n}}=n \pi+o(1)$.

Lemma 3.5. For any Dirichlet eigenvalue $\mu$ of $Q(v)$ with $v \in H_{c}^{1}$, $\operatorname{Mult}_{a}(\mu)=\operatorname{Mult}\left(\mu, \chi_{D}\right)$.

Proof. The algebraic multiplicity $\operatorname{Mult}_{a}(\mu)$ of $\mu$ equals the dimension of the range of the Riesz projector

$$
\Pi(\mu):=\frac{1}{2 \pi \mathrm{i}} \int_{\Gamma(\mu)}\left(\lambda-Q_{\operatorname{dir}}(v)\right)^{-1} \mathrm{~d} \lambda,
$$

where $\Gamma(\mu)$ is a counterclockwise oriented contour around $\mu$ so that all Dirichlet eigenvalues of $Q(v)$ except $\mu$ are outside of $\Gamma(\mu)$. Since $\left(\lambda-Q_{\operatorname{dir}}(v)\right)^{-1}$ is a compact operator, $\operatorname{Mult}_{a}(\mu)$ is finite and $\operatorname{Mult}_{a}(\mu)=\operatorname{tr} \Pi(\mu)$. By Proposition 2.1 and Lemma 3.2, $\operatorname{Mult}_{a}\left(\mu_{n}\right)=\operatorname{Mult}_{a}\left(-\mu_{n}\right)$ and $\operatorname{Mult}\left(\mu_{n}, \chi_{D}\right)=$ $\operatorname{Mult}\left(-\mu_{n}, \chi_{D}\right)$. Hence it suffices to consider the Dirichlet eigenvalues in $\mathbb{C}^{+}$. By Lemma 3.3, the Dirichlet eigenvalues for $v=0$ contained in $\mathbb{C}^{+}$are given by $\mu_{k}^{0}=\frac{1}{2}\left(k \pi+\sqrt{k^{2} \pi^{2}+1 / 4}\right), k \in \mathbb{Z}$, and since $\chi_{D}(\lambda, 0)=\sin (\omega(\lambda))$ one has $\operatorname{Mult}\left(\mu_{k}^{0}, \chi_{D}\right)=1$. Note that for any $k \in \mathbb{Z}$,

$$
\left(\sin \left(\omega_{k}^{0} x\right), \cos \left(\omega_{k}^{0} x\right), \frac{1}{4 \mu_{k}^{0}} \sin \left(\omega_{k}^{0} x\right), \frac{1}{4 \mu_{k}^{0}} \cos \left(\omega_{k}^{0} x\right)\right)
$$

is an eigenfunction of $Q_{\text {dir }}(0)$, corresponding to the eigenvalue $\mu_{k}^{0}$, where $\omega_{k}^{0} \equiv$ $\omega\left(\mu_{k}^{0}\right)=\pi|k|$. Since $Q_{\operatorname{dir}}(0)$ is selfadjoint with respect to the canonical inner 
product on $L^{2}\left([0,1], \mathbb{C}^{4}\right)$, the algebraic multiplicity $\operatorname{Mult}_{a}\left(\mu_{k}^{0}\right)$ is one. Since $\operatorname{Mult}\left(\mu_{k}^{0}, \chi_{D}\right)=1$ it then follows that $\operatorname{Mult}_{a}\left(\mu_{k}^{0}\right)=\operatorname{Mult}\left(\mu_{k}^{0}, \chi_{D}\right)$ for any $k \in$ $\mathbb{Z}$. Now let $v_{0} \in H_{c}^{1}$ and consider the line segment $\left[0, v_{0}\right]$ from 0 to $v_{0}$ in $H_{c}^{1}$. Since it is compact it follows by the Counting Lemma that there exist a neighborhood $U$ of $\left[0, v_{0}\right]$ in $H_{c}^{1}$ and $N \geq 1$ such that for any potential $v$ in $U$ and $|k|>N, \mu_{k}(v) \in D_{k}$. It implies that $\operatorname{Mult}\left(\mu_{k}, \chi_{D}\right)=1$. Choosing $\Gamma\left(\mu_{n}\right):=\partial D_{n}$ as contour for the Riesz projector introduced above, one sees that $\operatorname{Mult}_{a}\left(\mu_{k}\right)=1$ for any $|k|>N$. For the remaining $4 N+2$ Dirichlet eigenvalues in $A_{N}$ consider the Riesz projector

$$
\Pi_{N}(v):=\frac{1}{2 \pi \mathrm{i}} \int_{\partial A_{N}}\left(\lambda-Q_{\mathrm{dir}}(v)\right)^{-1} \mathrm{~d} \lambda .
$$

Denote by $\mathcal{R}_{N}(v)$ the range of $\Pi_{N}(v)$ and let $\Lambda_{N}(v)=\left.Q(v)\right|_{\mathcal{R}_{N}}$. Since $\operatorname{tr} \Pi_{N}$ is continuous and hence constant in $U$, the dimension of $\mathcal{R}_{N}(v)$ is $4 N+2$ and $\Lambda_{N}$ maps $\mathcal{R}_{N}$ onto itself. Thus, $\xi_{N}(\lambda, v):=\operatorname{det}\left(\lambda-\Lambda_{N}(v)\right)$ is a polynomial of degree $4 N+2$. By construction, its roots are precisely the Dirichlet eigenvalues inside $A_{N}$, counted with their algebraic multiplicities. On the other hand, consider the polynomial

$$
\zeta_{N}(\lambda, v):=\prod_{|k| \leq N}\left(\lambda-\mu_{k}(v)\right)\left(\lambda+\mu_{k}(v),\right.
$$

formed by the roots $\mu_{k}(v),-\mu_{k}(v),|k| \leq N$, of $\chi_{D}$ inside $A_{N}$ counted with their multiplicities $\operatorname{Mult}\left(\mu_{k}, \chi_{D}\right)$. By the analyticity of $\chi_{D}$ and the argument principle, the coefficients of $\zeta_{N}$ are in fact analytic functions in $v \in U$. The same is true for the coefficients of $\xi_{N}$. By the same argument as in Lemma 3.4 there is a neighborhood $U^{(0)}$ of 0 in $H_{c}^{1}$ so that on $U^{(0)}, \mu_{k} \in D_{k}$ for any $k \in \mathbb{Z}$. Hence $\xi_{N}$ and $\zeta_{N}$ coincide on $U^{(0)} \cap U(\neq \emptyset)$. By the analyticity of the coefficients of $\zeta_{N}$ and $\xi_{N}$ we conclude that $\xi_{N}(\cdot, v)=\zeta_{N}(\cdot, v)$ for all $v \in U^{(0)} \cap U$, implying that on $U, \operatorname{Mult}_{a}\left(\mu_{k}(v)\right)=\operatorname{Mult}\left(\mu_{k}(v), \chi_{D}\right)$ for any $|k| \leq N$.

Denote by $Q_{\text {neu }}$ the operator $Q(v)$ with domain

$$
H_{\text {neu }}:=\left\{F=\left(F_{1}, F_{2}, F_{3}, F_{4}\right) \in H^{1}\left([0,1], \mathbb{C}^{4}\right): F_{2}(0)=0=F_{2}(1)\right\} .
$$

Its spectrum, referred to as Neumann spectrum, is discrete and coincides with the Neumann spectrum of the spectral problem (2.2), defined as the set of eigenvalues with eigenfunctions $f=\left(f_{1}, f_{2}\right) \in H^{1}\left([0,1], \mathbb{C}^{2}\right)$ such that $f_{2}(0)=0=f_{2}(1)$. Clearly, for any $v \in H_{c}^{1}, \nu \in \mathbb{C}^{*}$ is an eigenvalue of (2.2) if there exists $a \in \mathbb{C}^{*}$ such that

$$
\grave{M}(\lambda, v)\left(\begin{array}{l}
1 \\
0
\end{array}\right)=a\left(\begin{array}{l}
1 \\
0
\end{array}\right) \text {. }
$$

Since by Lemma 3.2 (ii), $\chi_{N}(\lambda, v)=-\chi_{D}(\lambda,-v)$, Theorem 3.1 and Lemma 3.4 yield the following results.

Theorem 3.6. The Neumann spectrum of $Q(v)$ with $v \in H_{c}^{1}$ is the zero set of the function $\chi_{N}(\lambda):=\grave{m}_{3}(\lambda),\left\{\nu \in \mathbb{C}^{*}: \chi_{N}(\nu)=0\right\}$. Furthermore, the 
multiplicity $\operatorname{Mult}\left(\nu, \chi_{N}\right)$ of the root $\nu$ equals the algebraic multiplicity $\operatorname{Mult}_{a}(\nu)$ of $\nu$ as a Neumann eigenvalue, i.e., the dimension of the (finite dimensional) vector space $\bigcup_{n>1} \operatorname{ker}\left(\nu-Q_{\mathrm{neu}}(v)\right)^{n}$. The function $\chi_{N}$ is antisymmetric in $\lambda$ and hence the Neumann spectrum is even in $\lambda$. The function $\chi_{N}$ is analytic and compact on $\mathbb{C}^{*} \times H_{c}^{1}$. For $v=0, \chi_{N}(\lambda, 0)=-\sin (\omega(\lambda))$. Finally, results corresponding to Lemma 3.4 also hold for the Neumann eigenvalues.

Lemma 3.3 and Lemma 3.2 (ii) lead to the following

Lemma 3.7. The Neumann spectrum of $Q(v)$ at $v=0$ coincides with the Dirichlet spectrum of $Q(v)$ at $v=0$.

The Neumann eigenvalues of $Q(v)$ for $v \in H_{c}^{1}$, contained in $\mathbb{C}^{+}$and counted with their algebraic multiplicities can be listed as a bi-infinite sequence

$$
0 \preceq \cdots \preceq \nu_{-2} \preceq \nu_{-1} \preceq \nu_{0} \preceq \nu_{1} \preceq \nu_{2} \preceq \cdots
$$

so that for $|k|$ sufficiently large, $\nu_{k}$ is the unique Neumann eigenvalue of $Q(v)$ in the disc $D_{k}$.

We finish this section with the following useful identity.

Lemma 3.8. For any Dirichlet or Neumann eigenvalue $\lambda$ of $Q(v)$ with $v \in$ $H_{c}^{1}$,

$$
\Delta^{2}(\lambda, v)-1=\delta^{2}(\lambda, v) .
$$

Proof. By the Wronskian identity $1=\grave{m}_{1} \grave{m}_{4}-\grave{m}_{2} \grave{m}_{3}$. Hence

$$
\Delta^{2}-1=\frac{1}{4}\left(\grave{m}_{1}+\grave{m}_{4}\right)^{2}-1
$$

satisfies

$$
\Delta^{2}-1=\frac{1}{4}\left(\grave{m}_{1}+\grave{m}_{4}\right)^{2}-\grave{m}_{1} \grave{m}_{4}+\grave{m}_{2} \grave{m}_{3}=\frac{1}{4}\left(\grave{m}_{1}-\grave{m}_{4}\right)^{2}+\grave{m}_{2} \grave{m}_{3}=\delta^{2}+\grave{m}_{2} \grave{m}_{3} .
$$

Since the Dirichlet and Neumann eigenvalues are roots of $\grave{m}_{2} \grave{m}_{3}$ the claimed identity follows.

Next we describe the periodic spectrum $\operatorname{spec}_{\text {per }}(Q)$ of the operator $Q=$ $Q_{1} \partial_{x}+Q_{0}$ with domain given by the subspace of functions $F$ in

$$
H_{\text {per } \pm}:=\left\{F \in H_{\text {loc }}^{1}\left(\mathbb{R}, \mathbb{C}^{4}\right): \forall x \in \mathbb{R} F(x+1)= \pm F(x)\right\} .
$$

It coincides with the periodic spectrum of the spectral problem (2.2). Hence a complex number $\lambda \in \mathbb{C}^{*}$ is in $\operatorname{spec}_{\text {per }}(Q)$ iff $\grave{M}(\lambda, v)$ has an eigenvalue \pm 1 . Since $\operatorname{det}(\grave{M})=1$, the eigenvalues $\xi_{ \pm}$of $\grave{M}(\lambda) \equiv \grave{M}(\lambda, v)$ satisfy

$$
0=\operatorname{det}\left(\xi_{ \pm} I-\grave{M}(\lambda)\right)=\xi_{ \pm}^{2}-2 \Delta(\lambda) \xi_{ \pm}+1
$$

and thus are given by

$$
\xi_{ \pm}=\Delta(\lambda) \pm \sqrt{\Delta^{2}(\lambda)-1} .
$$

Note that in (3.13), $\xi_{+}$and $\xi_{-}$are determined up to the choice of a branch of $\sqrt{\Delta^{2}(\lambda)-1}$. 
Theorem 3.9. The periodic spectrum of $Q(v)$ with $v \in H_{c}^{1}$ is discrete and coincides with the zero set $\left\{\lambda \in \mathbb{C}^{*}: \chi_{p}(\lambda, v)=0\right\}$ of the function $\chi_{p}(\lambda, v):=$ $\Delta^{2}(\lambda, v)-1$. Furthermore, the multiplicity of any root of $\chi_{p}$ coincides with its algebraic multiplicity as a periodic eigenvalue. By Lemma 2.14 (i) and (ii) the periodic spectrum is invariant under the involution $\lambda \rightarrow-\lambda$ and for any periodic eigenvalue of $Q(q, p), \frac{1}{16 \lambda}$ is a periodic eigenvalue of $Q(-q, p)$.

Proof. Let $v \in H_{c}^{1}$ be given. By (2.2), for any $\lambda \in \mathbb{C}^{*}$ and $F \in$ $H_{\text {loc }}^{1}\left(\mathbb{R}, \mathbb{C}^{4}\right)$, the identity $Q F=\lambda F$ is equivalent to $F=\left(f, \lambda^{-1} B f\right)$ where $f(x)=$ $M(x, \lambda, v) f(0)$. Hence the existence of a solution $F$ of $Q F=\lambda F$ with $F(1)=$ $\pm F(0)$ is equivalent to \pm 1 being an eigenvalue of $M(1, \lambda, v)$. By (3.13), \pm 1 is an eigenvalue of $M(1, \lambda, v)$ iff $\Delta(\lambda, v)= \pm 1$. This proves the characterization. The statement on the algebraic multiplicity of periodic eigenvalues is proved as the corresponding one for the Dirichlet eigenvalues (cf., Lemma 3.5) and hence we omit its proof.

For $v=0$ the periodic spectrum of $Q(v)$ can be computed explicitly. By Lemma 2.16, $\chi_{p}(\lambda, 0)=\cos ^{2}(\omega(\lambda))-1=-\sin ^{2}(\omega(\lambda))$, where we recall that $\omega(\lambda)=\lambda-\frac{1}{16 \lambda}$.

Corollary 3.10. The periodic eigenvalues of $Q(v)$ for $v=0$ are

$$
\left\{\frac{n \pi}{2}\left(\sqrt[+]{1+\frac{1}{4 n^{2} \pi^{2}}}+1\right), \quad \frac{n \pi}{2}\left(\sqrt[+]{1+\frac{1}{4 n^{2} \pi^{2}}}-1\right): n \neq 0\right\} \bigcup\left\{\frac{1}{4},-\frac{1}{4}\right\} .
$$

Each eigenvalue has algebraic multiplicity two.

It is convenient to list the two sequences of periodic eigenvalues of $Q(0)$ with their algebraic multiplicities as follows:

$$
\begin{aligned}
0<\cdots< & \lambda_{-1}^{-}=\lambda_{-1}^{+}<\lambda_{0}^{-}=\lambda_{0}^{+}=\frac{1}{4}<\lambda_{1}^{-}=\lambda_{1}^{+}<\lambda_{2}^{-}=\lambda_{2}^{+}<\cdots \\
& \cdots<-\lambda_{1}^{-}=-\lambda_{1}^{+}<-\lambda_{0}^{-}=-\lambda_{0}^{+}=-\frac{1}{4}<-\lambda_{-1}^{-}=-\lambda_{-1}^{+}<\cdots<0 .
\end{aligned}
$$

We note that

$$
\Delta\left(\lambda_{k}^{+}, 0\right)=\Delta\left(\lambda_{k}^{-}, 0\right)=(-1)^{k}, \quad k \in \mathbb{Z}, \quad \text { and } \quad \lambda_{-k}^{+}=\frac{1}{16 \lambda_{k}^{+}}, \quad k \geq 0 .
$$

The periodic spectrum of $Q(v)$ for arbitrary $v \in H_{c}^{1}$ is asymptotically close to the one of $Q(0)$.

Lemma 3.11 (Counting Lemma). For each potential in $H_{c}^{1}$, there exist a neighborhood $U$ in $H_{c}^{1}$ and an integer $N>0$ such that for every $v \in U$, the entire function $\chi_{p}(\lambda, v)$ has exactly two roots in each of the domains $D_{n},-D_{n}, D_{-n}$, and $-D_{-n}$ with $n>N$ and exactly $4+8 N$ roots in the annulus $A_{N}$, counted with their multiplicities. There are no further roots. 
Proof. By Lemma 2.17, $\chi_{p}(\lambda, v)=\chi_{p}(\lambda, 0)(1+o(1))$ for $|\lambda| \rightarrow \infty$ with $\lambda \notin$ $\bigcup_{n \geq 1} D_{n} \cup\left(-D_{n}\right)$, locally uniformly in $v \in H_{c}^{1}$. Hence, for any potential in $H_{c}^{1}$ there is a neighborhood $U$ and an integer $N \geq 1$ such that for any $v \in U$

$$
\begin{aligned}
\left|\chi_{p}(\lambda, v)-\chi_{p}(\lambda, 0)\right| & <\left|\chi_{p}(\lambda, 0)\right|, \\
\left|\chi_{p}(\lambda,-q, p)-\chi_{p}(\lambda, 0,0)\right| & <\left|\chi_{p}(\lambda, 0,0)\right|
\end{aligned}
$$

on the boundaries of the discs $D_{n},-D_{n}$, and $B_{n}$ (defined in (3.2)) for any $n \geq$ $N$. The estimate (3.16) implies by Lemma 2.14 that

$$
\begin{aligned}
\left|\chi_{p}\left(\frac{1}{16 \lambda}, q, p\right)-\chi_{p}\left(\frac{1}{16 \lambda}, 0,0\right)\right| & =\left|\chi_{p}(\lambda,-q, p)-\chi_{p}(\lambda, 0,0)\right| \\
& <\left|\chi_{p}(\lambda, 0,0)\right|=\left|\chi_{p}\left(\frac{1}{16 \lambda}, 0,0\right)\right| .
\end{aligned}
$$

It then follows that for any $n \geq N,(3.15)$ holds on the boundaries of $\pm D_{n}, \pm D_{-n}$ and $B_{n}, B_{-n}$ and hence on the boundary of $A_{n}=B_{n} \backslash B_{-n}$. By Rouché's theorem, $\chi_{p}(\cdot, v)$ then has as many roots inside any of the discs $\pm D_{n}, \pm D_{-n}$ and annuli $A_{n}$ as $\chi_{p}(\lambda, 0)$ for any $n \geq N$. Since $\left(A_{n}\right)_{n \geq N}$ covers $\mathbb{C}^{*}$ the same argument shows that $\chi_{p}(\lambda, v)$ has no roots in $\mathbb{C}^{*} \backslash\left(A_{N} \cup \bigcup_{n>N}\left(D_{n} \cup\left(-D_{n}\right) \cup D_{-n} \cup\left(-D_{-n}\right)\right)\right)$.

For $v \in H_{c}^{2}$ one can provide a bound $N$ of Lemma 3.11 in terms of $\|v\|_{2}$. By Lemma 2.14 (i) it is enough to consider the part of the periodic spectrum of $Q(v)$ in the half plane $\mathbb{C}^{+}$and by Lemma 3.11 the periodic eigenvalues in $\mathbb{C}^{+}$, counted with their algebraic multiplicities, can be listed as a bi-infinite sequence

$$
0 \preceq \cdots \preceq \lambda_{-1}^{-} \preceq \lambda_{-1}^{+} \preceq \lambda_{0}^{-} \preceq \lambda_{0}^{+} \preceq \lambda_{1}^{-} \preceq \lambda_{1}^{+} \preceq \cdots .
$$

Note that the segment $\{t v \in 1: t \in[0,1]\}$ connecting $v$ to 0 in $H_{c}^{1}$ is compact and hence the integer $N$ of Lemma 3.11 can be chosen uniformly in $0 \leq t \leq$ 1. Furthermore, for any $|k| \geq N, \Delta\left(\lambda_{k}^{+}(t v), t v\right)=\Delta\left(\lambda_{k}^{-}(t v), t v\right)$ and its sign is constant in $t$. We conclude that for such $k, \Delta\left(\lambda_{k}^{ \pm}, v\right)=(-1)^{k}$.

We finish this section by a discussion on the roots of $\dot{\Delta}(\lambda, v) \equiv \partial_{\lambda} \Delta(\lambda, v)$. Since $\Delta$ is even with respect to the variable $\lambda, \dot{\Delta}$ is odd and hence it is again enough to look at the roots of $\dot{\Delta}$ in $\mathbb{C}^{+}$. For $v=0$ one has $\Delta(\lambda) \equiv \Delta(\lambda, 0)=$ $\cos (\omega(\lambda))$, where $\omega(\lambda)=\lambda-\frac{1}{16 \lambda}$, and hence

$$
\dot{\Delta}(\lambda) \equiv \dot{\Delta}(\lambda, 0)=-\left(1+\frac{1}{16 \lambda^{2}}\right) \sin (\omega(\lambda)) .
$$

The roots of $\dot{\Delta}(\lambda)$ in $\mathbb{C}^{+}$are given by the set of complex numbers consisting of the bi-infinite sequence $\dot{\lambda}_{k} \equiv \dot{\lambda}_{k}(0)=\lambda_{k}^{+}(0), k \in \mathbb{Z}$, and the additional root $\dot{\lambda}_{*}=\frac{i}{4}$. Each of these roots has multiplicity one. By Lemma 2.14 (ii), one has $-\frac{1}{16 \lambda^{2}} \dot{\Delta}\left(\frac{1}{16 \lambda}, q, p\right)=\dot{\Delta}(\lambda,-q, p)$. Since $-\frac{1}{16 \lambda^{2}} \dot{\Delta}\left(\frac{1}{16 \lambda}, q, p\right)$ and $\dot{\Delta}\left(\frac{1}{16 \lambda}, q, p\right)$ have the same roots in $\mathbb{C}^{*}$ (counted with their multiplicities), we can use the same arguments as for the periodic eigenvalues of $Q(v)$, to prove the following: 
Lemma 3.12 (Counting Lemma). Given any potential in $H_{c}^{1}$, there exists a neighborhood $U$ of it in $H_{c}^{1}$ and $N>0$ ( $U$ and $N$ can be chosen as in Lemma 3.11) so that for any $v \in U$, the function $\lambda \mapsto \dot{\Delta}(\lambda, v)$ has exactly one root in each of the domains $D_{n},-D_{n}, D_{-n}$, and $-D_{-n}$ with $n>N$ and $4+4 N$ roots in the annulus $A_{N}$. There are no other roots.

By this lemma, the roots of $\dot{\Delta}(\cdot, v)$ in $\mathbb{C}^{+} \backslash A_{N}$, counted with their algebraic multiplicities, can be listed as a bi-infinite sequence

$$
0 \preceq \cdots \preceq \dot{\lambda}_{-N-2} \preceq \dot{\lambda}_{-N-1} \preceq \dot{\lambda}_{N+1} \preceq \dot{\lambda}_{N+2} \preceq \cdots, \quad \dot{\lambda}_{k} \in D_{k}, \quad|k|>N
$$

such that any remaining root $\dot{\lambda}$ in $\mathbb{C}^{+}$satisfies $\dot{\lambda}_{-N-1} \preceq \dot{\lambda} \preceq \dot{\lambda}_{N+1}$.

We finish this section by establishing estimates for the periodic, Dirichlet, and Neumann eigenvalues of $Q(v)$. A first result concerns a priori bounds of the imaginary part of any of these eigenvalues.

Lemma 3.13. For any $v \in H_{c}^{2}$ and any periodic, Dirichlet, or Neumann eigenvalue $\lambda \in \mathbb{C}^{+}$,

$$
|\operatorname{Im} \lambda| \leq\|v\|_{2}+e^{\|q\|_{1}} .
$$

Proof. Let $v \in H_{c}^{2}$ and recall that $Q=Q_{1} \partial_{x}+Q_{0}$ with $Q_{1}, Q_{0}$ given by (1.4) and for any $F, G \in H^{1}\left([0,1], \mathbb{C}^{4}\right)$,

$$
\langle Q(v) F, G\rangle=\left[Q_{1} F \cdot G\right]_{0}^{1}+\langle F, Q(\bar{v}) G\rangle,
$$

where $\langle\cdot, \cdot\rangle$ denotes the $L^{2}$ inner product,

$$
\langle F, G\rangle=\int_{0}^{1} F(x) \cdot \overline{G(x)} \mathrm{d} x .
$$

On the domains (contained in $\left.H^{1}\left([0,1], \mathbb{C}^{4}\right)\right)$ of $Q$, corresponding to periodic, Dirichlet, or Neumann boundary conditions one has $\left[Q_{1} F \cdot G\right]_{0}^{1}=0$. In particular if $\lambda$ is a periodic, Dirichlet, or Neumann eigenvalue and $F$ a corresponding eigenfunction with $\langle F, F\rangle=1$ one has

$$
2 \mathrm{i} \operatorname{Im} \lambda=\lambda-\bar{\lambda}=\langle Q(v) F, F\rangle-\langle F, Q(v) F\rangle=\langle(Q(v)-Q(\bar{v})) F, F\rangle .
$$

Note that $Q(v)-Q(\bar{v})=2 \mathrm{i} \operatorname{Im} Q_{0}(v)$ and hence, by the Cauchy-Schwarz inequality and the normalization condition $\langle F, F\rangle=1$,

$$
|\langle(Q(v)-Q(\bar{v})) F, F\rangle| \leq\left\|2\left(\operatorname{Im} Q_{0}(v)\right) F\right\|_{L^{2}},
$$

where by (1.4) and (2.23)

$$
\left\|2\left(\operatorname{Im} Q_{0}(v)\right) F\right\|_{L^{2}} \leq \frac{1}{2}\left(\left\|\operatorname{Im} P p+q_{x}\right\|_{L^{\infty}}+\max _{ \pm}\left\|\operatorname{Im} e^{ \pm q / 2}\right\|_{L^{\infty}}\right) \leq\|v\|_{2}+e^{\|q\|_{1}} .
$$

Note that $\mu_{m}, \nu_{m}, \dot{\lambda}_{m}$ are close to $m \pi$ for $m \rightarrow \infty$. Our next aim is to obtain more precise asymptotics for these quantities. First we need to establish the following auxiliary result. 
Lemma 3.14. For any bi-infinite sequence of complex numbers $\left(\zeta_{n}\right)_{n} \subset \mathbb{C}^{*}$ satisfying $\zeta_{n}=n \pi+O(1)$ as $n \rightarrow \pm \infty$ one has

$$
\begin{aligned}
\left.\Delta\right|_{\lambda=\zeta_{n}} & =\cos \left(\zeta_{n}\right)+\ell_{n}^{2}, & \left.\dot{\Delta}\right|_{\lambda=\zeta_{n}} & =-\sin \left(\zeta_{n}\right)+\ell_{n}^{2}, \\
\left.\delta\right|_{\lambda=\zeta_{n}} & =\ell_{n}^{2}, & \left.\dot{\delta}\right|_{\lambda=\zeta_{n}} & =\ell_{n}^{2}, \\
\left.\chi_{D}\right|_{\lambda=\zeta_{n}} & =-\sin \left(\zeta_{n}\right)+\ell_{n}^{2}, & \left.\dot{\chi}_{D}\right|_{\lambda=\zeta_{n}} & =-\cos \left(\zeta_{n}\right)+\ell_{n}^{2} .
\end{aligned}
$$

These estimates hold uniformly on subsets of sequences $\left(\zeta_{n}\right)_{n}$ where $\left(\omega\left(\zeta_{n}\right)-n \pi\right)_{n}$ is bounded. If in fact $\zeta_{n}=n \pi+\ell_{n}^{2}$, then

$$
\sin \left(\zeta_{n}\right)=\ell_{n}^{2} \text { and } \cos \left(\zeta_{n}\right)=(-1)^{n}+\ell_{n}^{2},
$$

yielding in particular the sharper asymptotics

$$
\left.\Delta\right|_{\lambda=\zeta_{n}}=(-1)^{n}+\ell_{n}^{2} \text { and }\left.\dot{\Delta}\right|_{\lambda=\zeta_{n}}=\ell_{n}^{2} \quad \text { for } n \rightarrow \pm \infty .
$$

Proof. The stated asymptotics follow from Theorem 2.12 (iii) and (iv).

Lemma 3.15. For any $v \in H_{c}^{1}$, the roots of $\dot{\Delta}$ in $\mathbb{C}^{+}$have the following asymptotics as $n \rightarrow \infty$

$$
\dot{\lambda}_{n}=n \pi+\ell_{n}^{2}, \quad \frac{1}{16 \dot{\lambda}_{-n}}=n \pi+\ell_{n}^{2} .
$$

These estimates hold locally uniformly on $H_{c}^{1}$.

Proof. Since by Lemma $3.12, \dot{\lambda}_{n}=n \pi+O(1)$, it follows from Lemma 3.14 that

$$
0=\dot{\Delta}\left(\dot{\lambda}_{n}\right)=-\sin \left(\dot{\lambda}_{n}\right)+\ell_{n}^{2}
$$

or $\sin \left(\dot{\lambda}_{n}\right)=\ell_{n}^{2}$. Since by Lemma $3.12,\left|\dot{\lambda}_{n}-n \pi\right|<\pi / 3$ for $|n|$ large enough, one has

$$
\begin{aligned}
\left|\dot{\lambda}_{n}-n \pi\right| \cos (\pi / 3) & \leq\left|\dot{\lambda}_{n}-n \pi\right|\left|\int_{0}^{1} \cos \left(\left(\dot{\lambda}_{n}-n \pi\right) s+n \pi\right) \mathrm{d} s\right| \\
& =\left|\sin \left(\dot{\lambda}_{n}\right)-\sin (n \pi)\right|=\ell_{n}^{2},
\end{aligned}
$$

proving the first claimed asymptotics. They in turn yield the second ones by Lemma 2.14 (ii).

By the same arguments one can prove that similar results hold for the Dirichlet eigenvalues.

Lemma 3.16. For any $v \in H_{c}^{1}$, the Dirichlet eigenvalues of $Q(v)$ in $\mathbb{C}^{+}$have the following asymptotics as $n \rightarrow \infty$

$$
\mu_{n}=n \pi+\ell_{n}^{2} \quad \text { and } \quad \frac{1}{16 \mu_{-n}}=n \pi+\ell_{n}^{2} .
$$

These estimates hold locally uniformly in $H_{c}^{1}$. 
We will now use Lemma 3.15 and Lemma 3.16 to prove the following result for the periodic eigenvalues of $Q(v)$.

Lemma 3.17. For any $v \in H_{c}^{1}$, the periodic eigenvalues of $Q(v)$ in $\mathbb{C}^{+}$have the following asymptotics as $n \rightarrow \infty$

$$
\lambda_{n}^{ \pm}=n \pi+\ell_{n}^{2} \quad \text { and } \quad \frac{1}{16 \lambda_{-n}^{ \pm}}=n \pi+\ell_{n}^{2}
$$

These estimates hold locally uniformly on $H_{c}^{1}$.

Proof. Let $v \in H_{c}^{1}$ be given. Since by Lemma 3.16, $\mu_{n}=n \pi+\ell_{n}^{2}$, Lemma 3.14 yields $\delta\left(\mu_{n}\right)=\ell_{n}^{2}$. Hence by Lemma 3.8 one has $\Delta^{2}\left(\mu_{n}\right)-1=\delta^{2}\left(\mu_{n}\right)=\ell_{n}^{1}$. On the other hand, Lemma 3.14 also yields that $\Delta\left(\mu_{n}\right)=(-1)^{n}+\ell_{n}^{2}$. Writing $\Delta^{2}\left(\mu_{n}\right)-1=\left(\Delta\left(\mu_{n}\right)-1\right)\left(\Delta\left(\mu_{n}\right)+1\right)$ the two latter estimates together imply that

$$
\Delta\left(\mu_{n}\right)=(-1)^{n}+\ell_{n}^{1}
$$

A similar estimate holds for $\Delta\left(\dot{\lambda}_{n}\right)$. Indeed, since $\dot{\lambda}_{n}-\mu_{n}=\ell_{n}^{2}$ by Lemma 3.15 and Lemma 3.16,

$$
\Delta\left(\dot{\lambda}_{n}\right)-\Delta\left(\mu_{n}\right)=\left(\dot{\lambda}_{n}-\mu_{n}\right) \int_{0}^{1} \dot{\Delta}\left(t \dot{\lambda}_{n}+(1-t) \mu_{n}\right) \mathrm{d} t,
$$

and $\dot{\Delta}\left(t \dot{\lambda}_{n}+(1-t) \mu_{n}\right)=\ell_{n}^{2}$ uniformly in $0 \leq t \leq 1$ by Lemma 3.14, it follows that $\Delta\left(\dot{\lambda}_{n}\right)-\Delta\left(\mu_{n}\right)=\ell_{n}^{1}$. Together with (3.22) this yields $\Delta\left(\dot{\lambda}_{n}\right)=(-1)^{n}+\ell_{n}^{1}$. The latter estimates can be applied as follows. Since $\dot{\Delta}\left(\dot{\lambda}_{n}\right)=0$ one has $[(1-$ t) $\left.\dot{\Delta}\left(t \lambda_{n}^{ \pm}+(1-t) \dot{\lambda}_{n}\right)\right]_{0}^{1}=0$ and therefore integrating by parts,

$$
\begin{aligned}
\Delta\left(\lambda_{n}^{ \pm}\right)-\Delta\left(\dot{\lambda}_{n}\right) & =\left(\lambda_{n}^{ \pm}-\dot{\lambda}_{n}\right) \int_{0}^{1} \dot{\Delta}\left(t \lambda_{n}^{ \pm}+(1-t) \dot{\lambda}_{n}\right) \mathrm{d} t \\
& =\left(\lambda_{n}^{ \pm}-\dot{\lambda}_{n}\right)^{2} \int_{0}^{1}(1-t) \ddot{\Delta}\left(t \lambda_{n}^{ \pm}+(1-t) \dot{\lambda}_{n}\right) \mathrm{d} t .
\end{aligned}
$$

Hence

$$
\left(\lambda_{n}^{ \pm}-\dot{\lambda}_{n}\right)^{2} \int_{0}^{1}(1-t) \ddot{\Delta}\left(t \lambda_{n}^{ \pm}+(1-t) \dot{\lambda}_{n}\right) \mathrm{d} t=\ell_{n}^{1} .
$$

Since $\Delta$ is analytic in $\lambda$ and $\dot{\Delta}\left(\zeta_{n}\right)=-\sin \left(\zeta_{n}\right)+\ell_{n}^{2}$ by Lemma 3.14, Cauchy's estimate yields

$$
\ddot{\Delta}\left(t \lambda_{n}^{ \pm}+(1-t) \dot{\lambda}_{n}\right)=-\cos \left(t \lambda_{n}^{ \pm}+(1-t) \dot{\lambda}_{n}\right)+\ell_{n}^{2}
$$

uniformly in $0 \leq t \leq 1$. For $n$ sufficiently large, $t \lambda_{n}^{ \pm}+(1-t) \dot{\lambda}_{n}$ is in $D_{n}$ and hence

$$
\int_{0}^{1}(1-t) \ddot{\Delta}\left(t \lambda_{n}^{ \pm}+(1-t) \dot{\lambda}_{n}\right) \mathrm{d} t
$$

is uniformly bounded away from zero for such $n$. So (3.23) yields $\lambda_{n}^{ \pm}-\dot{\lambda}_{n}=\ell_{n}^{2}$. Since by Lemma $3.15, \dot{\lambda}_{n}=n \pi+\ell_{n}^{2}$, the first claimed asymptotics follow. Those then yield the second ones by Lemma 2.14 (ii). 


\section{Proofs of Theorem 1.1 and Theorem 1.2}

In this section we prove Theorem 1.1 and Theorem 1.2. Actually, we prove stronger versions of them as stated in Theorem 4.10 and Theorem 4.15 below. For any potential $v$ in $H_{c}^{1}$ let

$$
G_{n} \equiv G_{n}(v):=\left[\lambda_{n}^{-}, \lambda_{n}^{+}\right]:=\left\{(1-t) \lambda_{n}^{-}+t \lambda_{n}^{+}: 0 \leq t \leq 1\right\}, \quad n \in \mathbb{Z} .
$$

By a slight abuse of terminology we refer to $G_{n}$ as the $n$th closed spectral gap, although if $v$ is not real valued it lacks a spectral interpretation. Furthermore for any $v \in H_{c}^{1}$ and $n \in \mathbb{Z}$ we introduce the gap length

$$
\gamma_{n}(v):=\lambda_{n}^{+}(v)-\lambda_{n}^{-}(v), \quad n \in \mathbb{Z} .
$$

Note that in general, $\gamma_{n}(v)$ is a complex number, but in case $v$ is real valued, it is real and equals the length of the gap $G_{n}(v)$. For $d \geq 1$ and $s \in \mathbb{R}_{\geq 0}$, denote by $H^{s}\left(\mathbb{T}_{2}, \mathbb{C}^{d}\right)$ the Sobolev space of order $s$ of two periodic functions with values in $\mathbb{C}^{d}$,

$$
\begin{aligned}
& H^{s}\left(\mathbb{T}_{2}, \mathbb{C}^{d}\right):=\left\{u=\sum_{n \in \mathbb{Z}} u_{n} e_{n}: u_{n} \in \mathbb{C}^{d} \text { and }\|u\|_{s}<\infty\right\}, \\
&\|u\|_{s}:=\left(\sum_{n \in \mathbb{Z}}\langle n\rangle^{2 s}\left|u_{n}\right|^{2}\right)^{1 / 2},
\end{aligned}
$$

where $\mathbb{T}_{2}=\mathbb{R} / 2 \mathbb{Z}, e_{n}(x)=e^{\mathrm{i} n \pi x}$, and $|a|=\left(\sum_{j=1}^{d}\left|a_{j}\right|^{2}\right)^{1 / 2}$ for any $a=$ $\left(a_{1}, \ldots, a_{d}\right) \in \mathbb{C}^{d}$. We recall that the weights $\langle n\rangle^{s}:=\left(1+\pi^{2} n^{2}\right)^{s / 2}$ are submultiplicative for any $s \geq 0$, i.e., $\langle n+m\rangle^{s} \leq\langle n\rangle^{s}\langle m\rangle^{s}$. The $L^{2}$-inner product is defined for $f, g \in H^{0}\left(\mathbb{T}_{2}, \mathbb{C}^{d}\right) \equiv L^{2}\left(\mathbb{T}_{2}, \mathbb{C}^{d}\right)$ by

$$
\langle f, g\rangle_{c}=\frac{1}{2} \int_{0}^{2} f \bar{g} \mathrm{~d} x=\frac{1}{2} \int_{0}^{2} \sum_{j=1}^{d} f^{(j)}(x) \overline{g^{(j)}(x)} \mathrm{d} x,
$$

where $f^{(j)}, 1 \leq j \leq d$, denote the components of $f$. For a scalar valued function $u \in H^{s}\left(\mathbb{T}_{2}, \mathbb{C}\right)$ and a vector valued function $v \in H^{s}\left(\mathbb{T}_{2}, \mathbb{C}^{d}\right)$ with $v=\sum_{n \in \mathbb{Z}} v_{n} e_{n}$ and $v_{n} \in \mathbb{C}^{d}$ one has

$$
\|u v\|_{s} \leq\|u\|_{s} \sum_{n \in \mathbb{Z}}\langle n\rangle^{s}\left|v_{n}\right|
$$

Hence by the Cauchy-Schwarz inequality, $\sum_{n \in \mathbb{Z}} \frac{1}{\langle n\rangle^{2}} \leq 1+\frac{2}{\pi^{2}} \sum_{n=1}^{\infty} \frac{1}{n^{2}}$, and since

$$
\sum_{n=1}^{\infty} \frac{1}{n^{2}}=\frac{\pi^{2}}{6}
$$

one has

$$
\|u v\|_{s} \leq 2\|u\|_{s}\|v\|_{s+1} .
$$


Recall that by $(2.5),(2.6), \mathcal{Q}(q, p)=\mathcal{Q}_{1} \partial_{x}+\mathcal{Q}_{0}(q, p)$ with

$$
\mathcal{Q}_{1}=\left(\begin{array}{l}
R \\
\end{array}\right), \quad \mathcal{Q}_{0}(q, p)=\left(\begin{array}{ll}
\mathcal{A}(q, p) & \mathcal{B}(q, p) \\
\mathcal{B}(q, p) &
\end{array}\right)
$$

and

$$
\mathcal{A}(q, p)=\frac{1}{4} \varphi J, \varphi:=-\mathrm{i}\left(P p+q_{x}\right), \quad \mathcal{B}(q, p)=\frac{1}{4}\left(\begin{array}{cc}
\cosh (q / 2) & -\sinh (q / 2) \\
-\sinh (q / 2) & \cosh (q / 2)
\end{array}\right) .
$$

It turns out to be useful to introduce the linear isomorphism $H_{\mathbb{C}}^{s+1} \times H_{\mathbb{C}}^{s+1} \rightarrow$ $H_{\mathbb{C}}^{s+1} \times H_{\mathbb{C}}^{s},(q, p) \mapsto(q, \varphi)$ and use $(q, \varphi)$ instead of $(q, p)$ as phase space variables. Furthermore introduce

$$
\tilde{H}_{c}^{s+1}:=H_{\mathbb{C}}^{s+1} \times H_{\mathbb{C}}^{s}
$$

and by a slight abuse of notation we write $\mathcal{Q} \equiv \mathcal{Q}(q, \varphi)$ and $\mathcal{Q}_{0} \equiv \mathcal{Q}_{0}(q, \varphi)$ for the operators $\mathcal{Q}(q, p)$ and $\mathcal{Q}_{0}(q, p)$ respectively. We rewrite equation $(2.11)$ in the form

$$
\mathcal{Q}(\lambda) F=\mathcal{Q}_{0} F, \quad \mathcal{Q}(\lambda):=-\mathcal{Q}_{1} \partial_{x}+\lambda I
$$

and introduce the $L^{2}$-orthogonal basis of $L^{2}\left(\mathbb{T}_{2}, \mathbb{C}^{4}\right)$,

$$
e_{n}^{(j)}(x)=e_{n}(x) a^{(j)}, \quad e_{n}(x)=e^{\mathrm{i} n \pi x}, \quad \forall 1 \leq j \leq 4, n \in \mathbb{Z},
$$

where $a^{(1)}, a^{(2)}, a^{(3)}, a^{(4)}$ denote the standard basis in $\mathbb{C}^{4}, a^{(1)}=(1,0,0,0), a^{(2)}=$ $(0,1,0,0), a^{(3)}=(0,0,1,0), a^{(4)}=(0,0,0,1)$. Note that

$$
\begin{array}{ll}
\mathcal{Q}(\lambda) e_{n}^{(1)}=(\lambda+n \pi) e_{n}^{(1)}, & \mathcal{Q}(\lambda) e_{n}^{(2)}=(\lambda-n \pi) e_{n}^{(2)}, \\
\mathcal{Q}(\lambda) e_{n}^{(3)}=\lambda e_{n}^{(3)}, & \mathcal{Q}(\lambda) e_{n}^{(4)}=\lambda e_{n}^{(4)},
\end{array}
$$

suggesting to decompose $H^{s}\left(\mathbb{T}_{2}, \mathbb{C}^{4}\right)$ with $s \geq 0$ for any given $n \in \mathbb{Z}$ as $H^{s}\left(\mathbb{T}_{2}, \mathbb{C}^{4}\right)=\mathcal{P}_{n} \oplus \mathcal{K}_{n}$, where

$$
\begin{aligned}
& \mathcal{P}_{n}:=\left\{f_{-n}^{(1)} e_{-n}^{(1)}+f_{n}^{(2)} e_{n}^{(2)}: f_{-n}^{(1)}, f_{n}^{(2)} \in \mathbb{C}\right\} \\
& \mathcal{K}_{n}:=\left\{\sum_{k \in \mathbb{Z}, 1 \leq j \leq 4} f_{k}^{(j)} e_{k}^{(j)} \in H^{s}\left(\mathbb{T}_{2}, \mathbb{C}^{4}\right): f_{k}^{(j)} \in \mathbb{C}, f_{-n}^{(1)}=0, f_{n}^{(2)}=0\right\} .
\end{aligned}
$$

Denote the $L^{2}$-orthogonal projections onto $\mathcal{P}_{n}$ and $\mathcal{K}_{n}$ by $P_{n}$ and $K_{n}$, respectively. The subspaces $\mathcal{P}_{n}$ and $\mathcal{K}_{n}$ are invariant under $\mathcal{Q}(\lambda)$. Furthermore, introduce for any $n \in \mathbb{Z}$ the complex strip

$$
\Pi_{n}=\{\lambda \in \mathbb{C}:|\mathfrak{R} \lambda-n \pi| \leq \pi / 2\} .
$$

Note that these strips cover $\mathbb{C}$ and that for any $n \neq 0$, the restriction of $\mathcal{Q}(\lambda)$ to $\mathcal{K}_{n}$, again denoted by $\mathcal{Q}(\lambda)$, is invertible for any $\lambda \in \Pi_{n}$. Writing $F=u+v$ with $u:=P_{n} F$ and $v:=K_{n} F$, equation (4.8) decomposes into the following system of equations

$$
\mathcal{Q}(\lambda) u=P_{n} \mathcal{Q}_{0}(u+v),
$$




$$
\mathcal{Q}(\lambda) v=K_{n} \mathcal{Q}_{0}(u+v),
$$

called $P$ - and, respectively, $K$-equation. (Note that in this section, $u$ and $v$ have a different meaning than elsewhere in the paper.) Given any $n \neq 0$ we first solve the $K$-equation for any given $u \in \mathcal{P}_{n}$ and then substitute the solution into the $P$-equation, leading to a $2 \times 2$ system of linear equations with a $2 \times 2$ coefficient matrix $S_{n}$, which is singular precisely when $\lambda$ is a periodic eigenvalue of $\mathcal{Q}$.

Actually, to solve (4.12), it suffices to determine $\mathcal{Q}_{0} v$. Hence in a first step, we derive from (4.13) an equation for $\mathcal{Q}_{0} v$ instead of $v$. Once $u$ and $\mathcal{Q}_{0} v$ are found, $v$ can be easily determined from (4.13), $v=\mathcal{Q}(\lambda)^{-1} K_{n}\left(\mathcal{Q}_{0} u+\mathcal{Q}_{0} v\right)$. We begin by deriving from (4.13) an equation for $\mathcal{Q}_{0} v$. Given any $n \neq 0$ and $\lambda \in \Pi_{n}$, apply the operator $\mathcal{Q}_{0} \mathcal{Q}(\lambda)^{-1}$ to (4.13) to obtain

$$
\mathcal{Q}_{0} v=\mathcal{Q}_{0} \mathcal{Q}(\lambda)^{-1} K_{n} \mathcal{Q}_{0}(u+v),
$$

which leads to the following equation for $\tilde{v}=\mathcal{Q}_{0} v \in L^{2}\left(\mathbb{T}_{2}, \mathbb{C}^{4}\right)$,

$$
\left(I d-T_{n}\right) \tilde{v}=T_{n} \mathcal{Q}_{0} u, \quad T_{n}:=\mathcal{Q}_{0} \mathcal{Q}(\lambda)^{-1} K_{n}: L^{2}\left(\mathbb{T}_{2}, \mathbb{C}^{4}\right) \rightarrow L^{2}\left(\mathbb{T}_{2}, \mathbb{C}^{4}\right) .
$$

We then prove that for $|n|$ sufficiently large, $T_{n}^{4}$ is a contraction implying that for such $n, I d-T_{n}^{4}$ is invertible. The invertibility of the operator $I d-T_{n}$ then follows from the identity

$$
\left(I d-T_{n}\right)^{-1}=\left(I d+T_{n}\right)\left(I d+T_{n}^{2}\right)\left(I d-T_{n}^{4}\right)^{-1} .
$$

First we need to introduce some more notation. For $d=1,2,4$ and $s \geq 0$ we consider on $H^{s}\left(\mathbb{T}_{2}, \mathbb{C}^{d}\right)$ the shifted norms

$$
\|u\|_{s ; n}:=\left\|u e_{n}\right\|_{s}=\left(\sum_{k \in \mathbb{Z}}\langle k+n\rangle^{2 s}\left|u_{n}\right|^{2}\right)^{1 / 2} .
$$

Note that the estimate (4.6) continues to be valid for these norms. More precisely, the following holds:

Lemma 4.1. Let $s \in \mathbb{R}_{\geq 0}$ and $n \in \mathbb{Z}$. Then the following holds:

(i) For any $u \in H^{s}\left(\mathbb{T}_{2}, \mathbb{C}\right), v \in H^{s+1}\left(\mathbb{T}_{2}, \mathbb{C}^{d}\right)$ :

$$
\|u v\|_{s ; n} \leq 2\|u\|_{s ; n}\|v\|_{s+1}, \quad\|u v\|_{s ; n} \leq 2\|u\|_{s}\|v\|_{s+1 ; n} .
$$

(ii) For any $u \in H^{s+1}\left(\mathbb{T}_{2}, \mathbb{C}\right), v \in H^{s}\left(\mathbb{T}_{2}, \mathbb{C}^{d}\right)$ :

$$
\|u v\|_{s ; n} \leq 2\|u\|_{s+1 ; n}\|v\|_{s}, \quad\|u v\|_{s ; n} \leq 2\|u\|_{s+1}\|v\|_{s ; n} .
$$

Proof. One computes

$$
\|u v\|_{s ; n}=\left\|u v e_{n}\right\|_{s} \leq 2\left\|u e_{n}\right\|_{s}\|v\|_{s+1}=2\|u\|_{s ; n}\|v\|_{s+1} .
$$

The other inequalities are obtained in a similar fashion. 
Lemma 4.2. For any $(q, \varphi) \in \tilde{H}_{c}^{s+1}$ with $s \geq 0, l \in \mathbb{Z}$, and $\lambda \in \Pi_{n}$ with $n \neq$ 0 , the following holds:

(i) Decomposing

$$
T_{n}=\mathcal{Q}_{0} \mathcal{Q}(\lambda)^{-1} K_{n}:\left(H^{s}\left(\mathbb{T}_{2}, \mathbb{C}^{4}\right),\|\cdot\|_{s ; l}\right) \rightarrow\left(H^{s}\left(\mathbb{T}_{2}, \mathbb{C}^{4}\right),\|\cdot\|_{s ; l}\right)
$$

according to

$$
\mathcal{Q}_{0}=\left(\begin{array}{l}
\mathcal{A} \\
\end{array}\right)+\left(\mathcal{B}_{\mathcal{B}}\right)+\left(\begin{array}{l}
\mathcal{B} \\
\end{array}\right)
$$

the resulting operators satisfy

$$
\begin{aligned}
& \left\|\left(\begin{array}{c}
\mathcal{A} \\
)
\end{array}\right) \mathcal{Q}(\lambda)^{-1} K_{n}\right\|_{s ; l} \leq\|\varphi\|_{s}, \\
& \left\|\left(\begin{array}{c}
\mathcal{B} \\
)
\end{array}\right) \mathcal{Q}(\lambda)^{-1} K_{n}\right\|_{s ; l} \leq\|\sinh (q / 2)\|_{s}+\|\cosh (q / 2)\|_{s}, \\
& \left\|\left(\begin{array}{c}
\mathcal{B} \\
\end{array}\right) \mathcal{Q}(\lambda)^{-1} K_{n}\right\|_{s ; l} \leq \frac{\|\sinh (q / 2)\|_{s+1}+\|\cosh (q / 2)\|_{s+1}}{|n|} .
\end{aligned}
$$

(ii) $T_{n}$ is bounded. More precisely, $\left\|T_{n}\right\|_{s ; l} \leq R_{s}$, where

$$
R_{s} \equiv R_{s}(q, \varphi):=\|\varphi\|_{s}+\|\sinh (q / 2)\|_{s+1}+\|\cosh (q / 2)\|_{s+1} .
$$

Proof. Let $n \neq 0$. Clearly (4.18) follows from (4.15)-(4.17). The latter estimates are proved separately. To prove (4.15) note that for $m \neq n$

$$
\min _{\lambda \in \Pi_{n}}|\lambda-m \pi| \geq|n-m| \geq 1
$$

implying that for any $\lambda \in \Pi_{n}$, the restriction of $\mathcal{Q}(\lambda)$ to the invariant subspace $\mathcal{K}_{n}$ is invertible and that its inverse is uniformly bounded for $\lambda \in \Pi_{n}$. For any $F=\sum_{m \in \mathbb{Z}, j=1,2,3,4} f_{m}^{(j)} e_{m}^{(j)}$ with $f_{m}^{(j)} \in \mathbb{C}$, and $\lambda \in \Pi_{n}$

$$
\mathcal{Q}(\lambda)^{-1} K_{n} F=\sum_{m \neq n} \frac{1}{\lambda-m \pi}\left(f_{-m}^{(1)} e_{-m}^{(1)}+f_{m}^{(2)} e_{m}^{(2)}\right)+\frac{1}{\lambda} \sum_{m \in \mathbb{Z}}\left(f_{m}^{(3)} e_{m}^{(3)}+f_{m}^{(4)} e_{m}^{(4)}\right)
$$

is well defined. Taking into account Lemma 4.1 and definition (2.7) of $\mathcal{A}$ one has

$$
\begin{aligned}
\left\|(\mathcal{A}) \mathcal{Q}(\lambda)^{-1} \mathcal{K}_{n} F\right\|_{s ; l} & =\left\|(\mathcal{A}) \sum_{m \neq n} \frac{1}{\lambda-m \pi}\left(f_{-m}^{(1)} e_{-m}^{(1)}+f_{m}^{(2)} e_{m}^{(2)}\right)\right\|_{s ; l} \\
& \leq \frac{1}{4}\|\varphi\|_{s} \sum_{m \neq n} \frac{\left|f_{-m+l}^{(1)}\right|\langle-m+l\rangle^{s}+\left|f_{m+l}^{(2)}\right|\langle m+l\rangle^{s}}{|n-m|} .
\end{aligned}
$$

For $1 \leq j \leq 4$, let $f^{(j)}:=\sum_{m \in \mathbb{Z}} f_{m}^{(j)} e_{m}^{(j)}$. By the Cauchy-Schwarz inequality and (4.5) one then concludes 


$$
\begin{gathered}
\sum_{m \neq n} \frac{\left|f_{-m+l}^{(1)}\right|\langle-m+l\rangle^{s}+\left|f_{m+l}^{(2)}\right|\langle m+l\rangle^{s}}{|n-m|} \\
\quad \leq\left\|f^{(1)}+f^{(2)}\right\|_{s ; l}\left(\sum_{m \neq n} \frac{1}{|m-n|^{2}}\right)^{1 / 2} \leq \frac{\pi}{\sqrt{3}}\|F\|_{s ; l},
\end{gathered}
$$

where we used that

$$
\left\|f^{(1)}+f^{(2)}\right\|_{s ; l}^{2}=\left\|f^{(1)}\right\|_{s ; l}^{2}+\left\|f^{(2)}\right\|_{s ; l}^{2} \leq\|F\|_{s ; l}^{2} .
$$

This shows (4.15). A similar estimate holds for

$$
\left\|\left({ }_{\mathcal{B}}\right) \mathcal{Q}(\lambda)^{-1} \mathcal{K}_{n} F\right\|_{s ; l}
$$

where $\frac{1}{4}\|\varphi\|_{s}$ in the estimate above is replaced by $\frac{1}{4}\|\sinh (q / 2)\|_{s}+\frac{1}{4}\|\cosh (q / 2)\|_{s}$. This yields estimate (4.16). On the other hand,

$$
\left(\begin{array}{c}
\mathcal{B} \\
\end{array}\right) \mathcal{Q}(\lambda)^{-1} \mathcal{K}_{n} F=\left({ }^{\mathcal{B}}\right) \frac{1}{\lambda} \sum_{m \in \mathbb{Z}}\left(f_{m}^{(3)} e_{m}^{(3)}+f_{m}^{(4)} e_{m}^{(4)}\right) .
$$

Since by (4.19), $\frac{1}{|\lambda|} \leq \frac{1}{|n|}$ for any $\lambda \in \Pi_{n}$, estimate (4.17) then follows from Lemma 4.1.

Lemma 4.3. Let $(q, \varphi) \in \tilde{H}_{c}^{s+1}$ with $s \geq 0$ and $\lambda \in \Pi_{n}$ with $n \in \mathbb{Z} \backslash\{0\}$. Then the following holds:

(i) For any $F=\sum_{j=1}^{4} f^{(j)} \in H^{s}\left(\mathbb{T}_{2}, \mathbb{C}^{4}\right)$,

$$
\left\|T_{n}\left(\begin{array}{l}
\mathcal{A} \\
\end{array}\right) \mathcal{Q}(\lambda)^{-1} K_{n} f^{(1)}\right\|_{s ;-n} \leq \frac{1}{2|n|} R_{s}(q, \varphi)\|\varphi\|_{s}\left\|f^{(1)}\right\|_{s ;-n}
$$

and

$$
\left\|T_{n}\left(\begin{array}{l}
\mathcal{A} \\
\end{array}\right) \mathcal{Q}(\lambda)^{-1} K_{n} f^{(2)}\right\|_{s ; n} \leq \frac{1}{2|n|} R_{s}(q, \varphi)\|\varphi\|_{s}\left\|f^{(2)}\right\|_{s ; n}
$$

while for $j=3,4$

$$
T_{n}(\mathcal{A}) \mathcal{Q}(\lambda)^{-1} K_{n} f^{(j)}=0
$$

(ii) Furthermore

$$
\left\|\left[(\mathcal{A}) \mathcal{Q}(\lambda)^{-1} K_{n}\right]^{3}\right\|_{s ; \pm n} \leq \frac{1}{|n|}\|\varphi\|_{s}^{3} .
$$


(iii) For any $F=\sum_{j=1}^{4} f^{(j)} \in H^{s}\left(\mathbb{T}_{2}, \mathbb{C}^{4}\right)$,

$$
\left\|T_{n}\left(\begin{array}{l}
\mathcal{A} \\
\end{array}\right) \mathcal{Q}(\lambda)^{-1} K_{n}\right\|_{s ; \pm n} \leq R_{s}(q, \varphi)\left(\frac{2}{\sqrt{|n|}}\|\varphi\|_{s}+R_{s ;|n|}(\varphi)\right),
$$

where for any $g=\sum_{k \in \mathbb{Z}} g_{k} e_{k} \in H^{s}\left(\mathbb{T}_{2}, \mathbb{C}\right)$,

$$
R_{s ;|n|}(g):=\left(\sum_{|k| \geq|n|}\langle k\rangle^{2 s}\left|g_{k}\right|^{2}\right)^{1 / 2} .
$$

Proof. (i) Writing $\varphi=\sum_{m \in \mathbb{Z}} \varphi_{m} e_{m}$, it is easy to see that for $F=\sum_{j=1}^{4} f^{(j)} \in$ $H^{s}\left(\mathbb{T}, \mathbb{C}^{4}\right)$

$$
\begin{aligned}
g^{(1)} & :=\mathcal{Q}(\lambda)^{-1} K_{n}(\mathcal{A}) \mathcal{Q}(\lambda)^{-1} K_{n} f^{(2)} \\
& =\frac{1}{4} \sum_{k \neq-n} \sum_{m \neq n} \frac{f_{m}^{(2)} \varphi_{k-m}}{(\lambda-m \pi)(\lambda+k \pi)} e_{k}^{(1)}
\end{aligned}
$$

and similarly

$$
\begin{aligned}
g^{(2)} & :=\mathcal{Q}(\lambda)^{-1} K_{n}(\mathcal{A}) \mathcal{Q}(\lambda)^{-1} K_{n} f^{(1)} \\
& =\frac{1}{4} \sum_{k \neq n} \sum_{m \neq-n} \frac{-f_{m}^{(1)} \varphi_{k-m}}{(\lambda+m \pi)(\lambda-k \pi)} e_{k}^{(2)},
\end{aligned}
$$

while for $j=3,4$

$$
\left(\begin{array}{l}
\mathcal{A} \\
\end{array}\right) \mathcal{Q}(\lambda)^{-1} K_{n} f^{(j)}=0
$$

Note that the coefficients of $g^{(1)}=\sum_{k \neq-n} g_{k}^{(1)} e_{k}^{(1)}$ and $g^{(2)}=\sum_{k \neq n} g_{k}^{(2)} e_{k}^{(2)}$ are given by

$$
g_{k}^{(1)}=\frac{1}{4} \sum_{m \neq n} \frac{f_{m}^{(2)} \varphi_{k-m}}{(\lambda-m \pi)(\lambda+k \pi)}, \quad g_{k}^{(2)}=-\frac{1}{4} \sum_{m \neq-n} \frac{f_{m}^{(1)} \varphi_{k-m}}{(\lambda+m \pi)(\lambda-k \pi)} .
$$

By Lemma 4.1, one has for $j=1,2$ and $i=1,2$ such that $\{1,2\}=\{i, j\}$

$$
\begin{aligned}
\left\|T_{n}\left(\begin{array}{l}
\mathcal{A} \\
\end{array}\right) \mathcal{Q}(\lambda)^{-1} K_{n} f^{(j)}\right\|_{s ; l} & =\left\|\mathcal{Q}_{0} g^{(i)}\right\|_{s ; l} \\
\leq & \frac{1}{4}\left(\|\varphi\|_{s}+\|\sinh (q / 2)\|_{s}+\|\cosh (q / 2)\|_{s}\right)\left\|g^{(i)} e_{l}\right\|_{W^{s, 1}}
\end{aligned}
$$

where

$$
\left\|g^{(i)}\right\|_{W^{s, 1}}:=\sum_{m \in \mathbb{Z}}\langle m\rangle^{s}\left|g_{m}^{(i)}\right|
$$


The bounds (4.20) and (4.21) then follow from corresponding bounds of $\left\|g^{(1)} e_{n}\right\|_{W^{s, 1}}$ and $\left\|g^{(2)} e_{-n}\right\|_{W^{s, 1}}$. Indeed,

$$
\begin{aligned}
\left\|g^{(1)} e_{n}\right\|_{W^{s, 1}} & =\left\|\frac{1}{4} \sum_{k \neq-n} \sum_{m \neq n} \frac{f_{m}^{(2)} \varphi_{k-m}}{(\lambda-m \pi)(\lambda+k \pi)} e_{k}^{(1)} e_{n}\right\|_{W^{s, 1}} \\
& \leq \frac{1}{4} \sum_{k \neq-n} \sum_{m \neq n} \frac{\langle k+n\rangle^{s}}{|n-m||n+k|}\left|f_{m}^{(2)}\right|\left|\varphi_{k-m}\right| .
\end{aligned}
$$

Since for $k \neq-n, \frac{\langle k+n\rangle^{s}}{|k+n|} \leq 4\langle k+n\rangle^{s-1}$ and $\langle k+n\rangle^{s-1} \leq\langle m+n\rangle^{s-1}\langle k-m\rangle^{s-1}$ one obtains by Young's inequality

$$
\frac{1}{4} \sum_{k \neq-n} \sum_{m \neq n} \frac{\langle k+n\rangle^{s}}{|n-m||n+k|}\left|f_{m}^{(2)}\right|\left|\varphi_{k-m}\right| \leq \sum_{m \neq n} \frac{\left|f_{m}^{(2)}\right|\langle m+n\rangle^{s-1}}{|n-m|} \sum_{k \in \mathbb{Z}}\langle k\rangle^{s-1}\left|\varphi_{k}\right|
$$

and by the Cauchy-Schwarz inequality,

$$
\begin{aligned}
\sum_{m \neq n} \frac{\left|f_{m}^{(2)}\right|\langle m+n\rangle^{s-1}}{|n-m|} & =\sum_{m \neq n} \frac{1}{\langle m+n\rangle|n-m|}\left|f_{m}^{(2)}\right|\langle m+n\rangle^{s} \\
& \leq\left\|f^{(2)}\right\|_{s ; n}\left(\sum_{m \neq n} \frac{1}{\langle m+n\rangle^{2}|n-m|^{2}}\right)^{1 / 2}
\end{aligned}
$$

For $n>0, \sum_{m \neq n} \frac{1}{|n-m|^{2}\langle m+n\rangle^{2}}$ is bounded by

$$
\frac{1}{\pi^{2} n^{2}}+\frac{1}{\pi^{2} n^{2}} \sum_{m<0} \frac{1}{m^{2}}+\sum_{m>0, m \neq n} \frac{1}{|n-m|^{2} \pi^{2} n^{2}} \leq \frac{1}{n^{2}}\left(\frac{1}{\pi^{2}}+\frac{3}{\pi^{2}} \sum_{m>0} \frac{1}{m^{2}}\right) .
$$

Since a similar statement holds for $n<0$ and

$$
\sum_{k \in \mathbb{Z}}\langle k\rangle^{s-1}\left|\varphi_{k}\right| \leq\left(\sum_{k \in \mathbb{Z}} \frac{1}{\langle k\rangle^{2}}\right)^{1 / 2}\left(\sum_{k \in \mathbb{Z}}\langle k\rangle^{2 s}\left|\varphi_{k}\right|^{2}\right)^{1 / 2} \leq\|\varphi\|_{s}\left(1+\frac{1}{3}\right)^{1 / 2}
$$

as well as $\sum_{n=1}^{\infty} \frac{1}{n^{2}}=\frac{\pi^{2}}{6}$ one has

$$
\left\|g^{(1)} e_{n}\right\|_{W^{s, 1}} \leq\|\varphi\|_{s} \frac{2}{|n|}\left\|f^{(2)}\right\|_{s ; n} .
$$

A similar computation yields

$$
\left\|g^{(2)} e_{-n}\right\|_{W^{s, 1}} \leq\|\varphi\|_{s} \frac{2}{|n|}\left\|f^{(1)}\right\|_{s ;-n} .
$$

This proves (4.21) and (4.20). 
(ii) To prove (4.22), note that by (4.30) the definition (4.25) of $g^{(1)}$ and the estimate (4.4)

$$
\left\|\left[\left(\begin{array}{l}
\mathcal{A} \\
\end{array}\right) \mathcal{Q}(\lambda)^{-1} K_{n}\right]^{2} f^{(2)}\right\|_{s ; \pm n}=\left\|(\mathcal{A}) g^{(1)}\right\|_{s ; \pm n} \leq \frac{1}{4}\|\varphi\|_{s}\left\|g^{(1)} e_{ \pm n}\right\|_{W^{s, 1}}
$$

and hence by (4.32)

$$
\left\|\left[\left(\begin{array}{l}
\mathcal{A} \\
\end{array}\right) \mathcal{Q}(\lambda)^{-1} K_{n}\right]^{2} f^{(2)}\right\|_{s ; \pm n} \leq \frac{1}{2|n|}\|\varphi\|_{s}^{2}\left\|f^{(2)}\right\|_{s ; \pm n} .
$$

It then follows by (4.15) that

$$
\begin{aligned}
& \left\|\left[\left(\begin{array}{l}
\mathcal{A} \\
\end{array}\right) \mathcal{Q}(\lambda)^{-1} K_{n}\right]^{3} f^{(2)}\right\|_{s ; \pm n} \leq\|\varphi\|_{s}\left\|\left[\left(\begin{array}{l}
\mathcal{A} \\
\end{array}\right) \mathcal{Q}(\lambda)^{-1} K_{n}\right]^{2} f^{(2)}\right\|_{s ; \pm n} \\
& \leq \frac{1}{2|n|}\|\varphi\|_{s}^{3}\left\|f^{(2)}\right\|_{s ; \pm n} .
\end{aligned}
$$

By the definition of $\mathcal{A}=\frac{1}{4} \varphi J$, all components of $(\mathcal{A}) \mathcal{Q}(\lambda)^{-1} K_{n} f^{(1)}$ vanish except the second one. Therefore (4.34) implies

$$
\left\|\left[(\mathcal{A}) \mathcal{Q}(\lambda)^{-1} K_{n}\right]^{3} f^{(1)}\right\|_{s ; \pm n} \leq \frac{1}{2|n|}\|\varphi\|_{s}^{2}\left\|\left(\begin{array}{l}
\mathcal{A} \\
\end{array}\right) \mathcal{Q}(\lambda)^{-1} K_{n} f^{(1)}\right\|_{s ; \pm n} .
$$

By (4.15) it then follows that

$$
\left\|\left[\left(\begin{array}{l}
\mathcal{A} \\
\end{array}\right) \mathcal{Q}(\lambda)^{-1} K_{n}\right]^{3} f^{(1)}\right\|_{s ; \pm n} \leq \frac{1}{2|n|}\|\varphi\|_{s}^{3}\left\|f^{(1)}\right\|_{s ; \pm n}
$$

In view of (4.27) the claimed estimate (4.22) then follows.

(iii) In view of item (i) and the definitions (4.25) and (4.26) of $g^{(1)}$ and $g^{(2)}$, it remains to bound $\left\|g^{(2)} e_{n}\right\|_{W^{s, 1}}$ and $\left\|g^{(1)} e_{-n}\right\|_{W^{s, 1}}$. One computes

$$
\begin{aligned}
\left\|g^{(2)} e_{n}\right\|_{W^{s, 1}} & =\left\|\frac{1}{4} \sum_{k \neq n} \sum_{m \neq-n} \frac{-f_{m}^{(1)} \varphi_{k-m}}{(\lambda+m \pi)(\lambda-k \pi)} e_{k}^{(2)} e_{n}\right\|_{W^{s, 1}} \\
& \leq \frac{1}{4} \sum_{k \neq n} \sum_{m \neq-n} \frac{\left|f_{m}^{(1)}\right|\left|\varphi_{k-m}\right|\langle k+n\rangle^{s}}{|n+m||n-k|} .
\end{aligned}
$$

Since $\langle k+n\rangle^{s} \leq\langle k-m\rangle^{s}\langle m+n\rangle^{s}$, one has

$$
\sum_{k \neq n} \sum_{m \neq-n} \frac{\left|f_{m}^{(1)}\right|\left|\varphi_{k-m}\right|\langle k+n\rangle^{s}}{|n+m||n-k|} \leq \sum_{k \neq n} \sum_{m \neq-n} \frac{\left|f_{m}^{(1)}\right|\langle m+n\rangle^{s}\left|\varphi_{k-m}\right|\langle k-m\rangle^{s}}{|n+m||n-k|} .
$$


We split the latter sum into three parts defined by the three sets of summation indices

$$
\{(k, m):|n-k|>|n| / 2\}, \quad\{(k, m):|n-k| \leq|n| / 2,|n+m|>|n| / 2\},
$$

and

$$
\{(k, m):|n-k| \leq|n| / 2,|n+m| \leq|n| / 2\}
$$

Let

$$
I:=\sum_{|n-k|>\frac{|n|}{2}} \sum_{m \neq-n} \frac{\left|f_{m}^{(1)}\right|\langle m+n\rangle^{s}\left|\varphi_{k-m}\right|\langle k-m\rangle^{s}}{|n+m||n-k|} .
$$

Then by the Cauchy-Schwarz inequality $I$ is bounded by

$$
C\left(\sum_{|n-k|>\frac{|n|}{2}} \sum_{m \neq-n}\left|f_{m}^{(1)}\right|^{2}\langle m+n\rangle^{2 s}\left|\varphi_{k-m}\right|^{2}\langle k-m\rangle^{2 s}\right)^{1 / 2}
$$

where

$$
C:=\left(\sum_{|n-k|>\frac{|n|}{2}} \sum_{m \neq-n} \frac{1}{|n-k|^{2}|n+m|^{2}}\right)^{1 / 2}
$$

and hence

$$
I \leq\left(\sum_{|n-k|>\frac{|n|}{2}} \frac{1}{|n-k|^{2}}\right)^{1 / 2}\left(\sum_{m \neq-n} \frac{1}{|n+m|^{2}}\right)^{1 / 2}\|\varphi\|_{s}\left\|f^{(1)}\right\|_{s ; n} .
$$

By a similar computation

$$
\begin{aligned}
I I & :=\sum_{1 \leq|n-k| \leq \frac{|n|}{2}} \sum_{|n+m|>\frac{|n|}{2}} \frac{\left|f_{m}^{(1)}\right|\langle m+n\rangle^{s}\left|\varphi_{k-m}\right|\langle k-m\rangle^{s}}{|n+m||n-k|} \\
& \leq\left(\sum_{1 \leq|n-k| \leq \frac{|n|}{2}} \frac{1}{|n-k|^{2}}\right)^{1 / 2}\left(\sum_{|n+m|>\frac{|n|}{2}} \frac{1}{|n+m|^{2}}\right)^{1 / 2}\|\varphi\|_{s}\left\|f^{(1)}\right\|_{s ; n} .
\end{aligned}
$$

Since

$$
\sum_{|l|>\frac{|n|}{2}} \frac{1}{l^{2}} \leq 2 \sum_{l>\frac{|n|}{2}} \frac{1}{l^{2}} \leq 2 \int_{\frac{|n|}{2}}^{\infty} \frac{1}{x^{2}} \mathrm{~d} x=\frac{4}{|n|},
$$

it then follows that

$$
\frac{1}{4} I+\frac{1}{4} I I \leq \frac{1}{2}\left(2 \frac{\pi^{2}}{6}\right)^{1 / 2}\left(\frac{4}{|n|}\right)^{1 / 2}\|\varphi\|_{s}\left\|f^{(1)}\right\|_{s ; n} \leq \frac{2}{\sqrt{|n|}}\|\varphi\|_{s}\left\|f^{(1)}\right\|_{s ; n} .
$$


Now let us turn to the sum

$$
I I I:=\sum_{1 \leq|n-k| \leq \frac{|n|}{2}} \sum_{1 \leq|n+m| \leq \frac{|n|}{2}} \frac{\left|f_{m}^{(1)}\right|\langle m+n\rangle^{s}\left|\varphi_{k-m}\right|\langle k-m\rangle^{s}}{|n+m||n-k|} .
$$

Since $|k-m| \geq 2|n|-|k-n|-|m+n| \geq|n|$ for $k, m$ with $|n-k| \leq \frac{|n|}{2}$ and $\mid m+$ $n \mid \leq \frac{|n|}{2}$, the sum $I I I$ is bounded by

$$
\begin{aligned}
\left(\sum_{1 \leq|n-k| \leq \frac{|n|}{2}} \frac{1}{|n-k|^{2}}\right)^{1 / 2}\left(\sum_{1 \leq|n+m| \leq \frac{|n|}{2}} \frac{1}{|n+m|^{2}}\right)^{1 / 2} \\
\times\left(\sum_{|k| \geq|n|}\left|\varphi_{k}\right|^{2}\langle k\rangle^{2 s}\right)^{1 / 2}\left\|f^{(1)}\right\|_{s ; n} \leq 2 \frac{\pi^{2}}{6} R_{s ; n}(\varphi)\left\|f^{(1)}\right\|_{s ; n}
\end{aligned}
$$

and hence

$$
\frac{1}{4} I I I \leq R_{s ; n}(\varphi)\left\|f^{(1)}\right\|_{s ; n} .
$$

Altogether, we thus have proved that

$$
\left\|g^{(2)} e_{n}\right\|_{W^{s, 1}} \leq \frac{2}{\sqrt{|n|}}\|\varphi\|_{s}\left\|f^{(1)}\right\|_{s ; n}+R_{s ;|n|}(\varphi)\left\|f^{(1)}\right\|_{s ; n} .
$$

Combining (4.32) and (4.36) one obtains the estimate

$$
\left\|T_{n}(\mathcal{A}) \mathcal{Q}(\lambda)^{-1} K_{n}\right\|_{s ; n} \leq R_{s}(q, \varphi)\left(\frac{2}{\sqrt{|n|}}\|\varphi\|_{s}+R_{s ;|n|}(\varphi)\right) .
$$

Similarly, one shows that

$$
\left\|g^{(1)} e_{-n}\right\|_{W^{s, 1}} \leq \frac{2}{\sqrt{|n|}}\|\varphi\|_{s}\left\|f^{(2)}\right\|_{s ;-n}+R_{s ;|n|}(\varphi)\left\|f^{(2)}\right\|_{s ;-n}
$$

and deduces

$$
\left\|T_{n}\left(\begin{array}{l}
\mathcal{A} \\
\end{array}\right) \mathcal{Q}(\lambda)^{-1} K_{n}\right\|_{s ;-n} \leq R_{s}(q, \varphi)\left(\frac{2}{\sqrt{|n|}}\|\varphi\|_{s}+R_{s ;|n|}(\varphi)\right) .
$$

This proves (4.23).

Decomposing $T_{n}$ as in Lemma 4.2, the following identities can be verified in a straightforward way.

Lemma 4.4. Let $(q, \varphi) \in \tilde{H}_{c}^{s+1}$ with $s \geq 0$ and $\lambda \in \Pi_{n}$ with $n \in \mathbb{Z} \backslash\{0\}$. Then

$$
\left(\begin{array}{l}
\mathcal{A} \\
\end{array}\right) \mathcal{Q}(\lambda)^{-1} K_{n}\left(\mathcal{B}_{\mathcal{B}}\right) \mathcal{Q}(\lambda)^{-1} K_{n}=0
$$




$$
\begin{aligned}
& \left({ }^{\mathcal{B}}\right) \mathcal{Q}(\lambda)^{-1} K_{n}\left({ }^{\mathcal{A}}\right) \mathcal{Q}(\lambda)^{-1} K_{n}=0, \\
& \left({ }_{\mathcal{B}}\right) \mathcal{Q}(\lambda)^{-1} K_{n}\left({ }_{\mathcal{B}}\right) \mathcal{Q}(\lambda)^{-1} K_{n}=0, \\
& \left({ }^{\mathcal{B}}\right) \mathcal{Q}(\lambda)^{-1} K_{n}\left({ }^{\mathcal{B}}\right) \mathcal{Q}(\lambda)^{-1} K_{n}=0 .
\end{aligned}
$$

Lemma 4.5. Let $(q, \varphi) \in \tilde{H}_{c}^{s+1}$ with $s \geq 0$ and $\lambda \in \Pi_{n}$ with $n \in \mathbb{Z} \backslash\{0\}$.

(i) There exists an absolute constant $C_{0} \geq 1$ so that

$$
\left\|T_{n}^{4}\right\|_{s ; \pm n} \leq \frac{C_{0}}{|n|} R_{s}^{4}, R_{s} \equiv R_{s}(q, \varphi):=\|\varphi\|_{s}+\|\cosh (q / 2)\|_{s+1}+\|\sinh (q / 2)\|_{s+1} .
$$

(ii) With $R_{s,|n|}(\varphi)$ given as in (4.24) one has

$$
\begin{aligned}
\left\|T_{n}^{2}\right\|_{s ; \pm n} \leq R_{s}(q, \varphi)\left(\frac{\|\sinh (q / 2)\|_{s+1}+\|\cosh (q / 2)\|_{s+1}}{|n|}\right. & \\
& \left.+\frac{2}{\sqrt{|n|}}\|\varphi\|_{s}+R_{s ;|n|}(\varphi)\right) .
\end{aligned}
$$

(iii) For any $F=\sum_{j=1}^{4} f^{(j)} \in H^{s}\left(\mathbb{T}_{2}, \mathbb{C}^{4}\right)$ with $f^{(1)}=0$, the following sharper estimate holds

$$
\left\|T_{n}^{2} F\right\|_{s ; n} \leq \frac{1}{|n|} R_{s}^{2}(q, \varphi)\|F\|_{s ; n} .
$$

(iv) For any $F=\sum_{j=1}^{4} f^{(j)} \in H^{s}\left(\mathbb{T}_{2}, \mathbb{C}^{4}\right)$ with $f^{(2)}=0$, one has

$$
\left\|T_{n}^{2} F\right\|_{s ;-n} \leq \frac{1}{|n|} R_{s}^{2}(q, \varphi)\|F\|_{s ;-n} .
$$

Remark 4.6. It follows from Lemma 4.5 (i) that $T_{n}^{4}$ is a $\frac{1}{2}$-contraction for $|n| \geq 2 C_{0} R_{s}^{4}$. In contrast, the estimate of Lemma 4.5 (ii) implies that, $T_{n}^{2}$ is a $\frac{1}{2}$-contraction for $|n| \geq N$ where $N$ can be chosen locally uniformly on $\tilde{H}_{\mathbb{C}}^{s+1}$.

Proof. (i) Decompose $T_{n}$ into three terms,

$$
T_{n}=\left(\begin{array}{l}
\mathcal{A} \\
\end{array}\right) \mathcal{Q}(\lambda)^{-1} K_{n}+\left({ }_{\mathcal{B}}\right) \mathcal{Q}(\lambda)^{-1} K_{n}+\left(\begin{array}{l}
\mathcal{B} \\
\end{array}\right) \mathcal{Q}(\lambda)^{-1} K_{n}
$$

By Lemma 4.4, $T_{n}^{4}$ consists of a sum of terms, each containing

$$
\left({ }^{\mathcal{B}}\right) \mathcal{Q}(\lambda)^{-1} K_{n} \quad \text { or } \quad\left[(\mathcal{A}) \mathcal{Q}(\lambda)^{-1} K_{n}\right]^{3}
$$

as a factor. Using (4.15), (4.17), and (4.22) one obtains the claimed estimate $\left\|T_{n}^{4}\right\|_{s ; n} \leq C_{0} R_{s}^{4} /|n|$. 
(ii) Note that by Lemma 4.3

$$
\left\|T_{n}^{2}\right\|_{s ; \pm n} \leq\left\|T_{n}\left(\begin{array}{c}
\mathcal{B} \\
\mathcal{B}
\end{array}\right) \mathcal{Q}(\lambda)^{-1} K_{n}\right\|_{s ; \pm n}+R_{s}(q, \varphi)\left(\frac{2}{\sqrt{|n|}}\|\varphi\|_{s}+R_{s ; n}(\varphi)\right)
$$

and by Lemma 4.2 and Lemma 4.4,

$$
\begin{aligned}
\left\|T_{n}\left(\begin{array}{l}
\mathcal{B}^{\mathcal{B}} \\
\end{array}\right) \mathcal{Q}(\lambda)^{-1} K_{n}\right\|_{s ; \pm n} \\
\quad \leq R_{s}(q, \varphi) \frac{\|\sinh (q / 2)\|_{s+1}+\|\cosh (q / 2)\|_{s+1}}{|n|}
\end{aligned}
$$

(iii) For any $F=\sum_{j=1}^{4} f^{(j)} \in H^{s}\left(\mathbb{T}_{2}, \mathbb{C}^{4}\right)$

$$
\left\|T_{n}^{2} F\right\|_{s ; n} \leq\left\|T_{n}\left({ }_{\mathcal{B}}{ }^{\mathcal{B}}\right) \mathcal{Q}(\lambda)^{-1} K_{n} F\right\|_{s ; n}+\left\|T_{n}\left(\begin{array}{l}
\mathcal{A} \\
\end{array}\right) \mathcal{Q}(\lambda)^{-1} K_{n} F\right\|_{s ; n} .
$$

If $f^{(1)}=0$ then by Lemma 4.3 (i)

$$
\left\|T_{n}\left(\begin{array}{l}
\mathcal{A} \\
\end{array}\right) \mathcal{Q}(\lambda)^{-1} K_{n} F\right\|_{s ; n} \leq \frac{1}{2|n|} R_{s}(q, \varphi)\|\varphi\|_{s}\left\|f^{(2)}\right\|_{s ; n} .
$$

Hence (4.39) yields (iii).

(iv) Arguing as in the proof of item (iii) one obtains (iv).

We now go back to the $K$ - and $P$-equation. Let $(q, \varphi) \in \tilde{H}_{c}^{s+1}$ be given. Instead of the $K$-equation (4.13) we consider (4.14) which by the definition of $T_{n}$ takes the form $\mathcal{Q}_{0} v=T_{n} \mathcal{Q}_{0}(u+v)$. Solving for $\mathcal{Q}_{0} v$ yields

$$
\left(I d-T_{n}\right) \mathcal{Q}_{0} v=T_{n} \mathcal{Q}_{0} u .
$$

By Lemma 4.5 (iv), $T_{n}^{4}$ is a $1 / 2$-contraction for any $n$ with $|n| \geq 2 C_{0} R_{s}^{4}$. It follows that for such $n,\left(I d-T_{n}^{4}\right)$ and hence $\left(I d-T_{n}\right)$ are invertible where the inverse of $\left(I d-T_{n}\right)$ is given by

$$
\widehat{T}_{n}:=\left(I d-T_{n}\right)^{-1}=\left(I d-T_{n}^{4}\right)^{-1}\left(I d+T_{n}+T_{n}^{2}+T_{n}^{3}\right) .
$$

By Lemma 4.2 for any $s \geq 0$ and $|n| \geq 2 C_{0} R_{s}^{4}$

$$
\left\|\widehat{T}_{n}\right\|_{s ; \pm n} \leq 2\left(1+R_{s}+R_{s}^{2}+R_{s}^{3}\right) \leq 2\left(1+R_{s}\right)^{3} .
$$

By (4.40), $Q_{0} v$ is given by $\mathcal{Q}_{0} v=\widehat{T}_{n} T_{n} \mathcal{Q}_{0} u$ and the $P$-equation (4.12) becomes

$$
\mathcal{Q}(\lambda) u=P_{n} \mathcal{Q}_{0} u+P_{n} \widehat{T}_{n} T_{n} \mathcal{Q}_{0} u .
$$

Since $I d+\widehat{T}_{n} T_{n}=\widehat{T}_{n}$ one is led to $0=\left(\mathcal{Q}(\lambda)-P_{n} \widehat{T}_{n} \mathcal{Q}_{0}\right) u$. Hence given any $|n| \geq 2 C_{0} R_{s}^{2}(q, \varphi), \lambda \in \Pi_{n}$ is a periodic eigenvalue of $\mathcal{Q}$ iff $\operatorname{det}\left(S_{n}(\lambda)\right)=0$, where $S_{n}(\lambda) \equiv S_{n}(\lambda, q, \varphi)$ is the map

$$
S_{n}(\lambda)=\left(\mathcal{Q}(\lambda)-P_{n} \widehat{T}_{n} \mathcal{Q}_{0}\right) P_{n}: \mathcal{P}_{n} \rightarrow \mathcal{P}_{n} .
$$


We now compute the matrix representation of $S_{n}$ with respect to the basis $\left[e_{-n}^{(1)}, e_{n}^{(2)}\right]$ of $\mathcal{P}_{n}$. By (4.9), the matrix representation $[Q(\lambda)]$ of $Q(\lambda)$ is given by

$$
[\mathcal{Q}(\lambda)]=\left(\begin{array}{ll}
\lambda-n \pi & \\
& \lambda-n \pi
\end{array}\right)
$$

and for any $|n| \geq 2 C_{0} R_{s}^{2}(q, \varphi)$, the one of $P_{n} \widehat{T}_{n} \mathcal{Q}_{0} P_{n}$ is given by

$$
\left(\begin{array}{ll}
a_{n}^{+}(\lambda) & b_{n}^{+}(\lambda) \\
b_{n}^{-}(\lambda) & a_{n}^{-}(\lambda)
\end{array}\right):=\left(\begin{array}{ll}
\left\langle\widehat{T}_{n} \mathcal{Q}_{0} e_{-n}^{(1)}, e_{-n}^{(1)}\right\rangle_{c} & \left\langle\widehat{T}_{n} \mathcal{Q}_{0} e_{n}^{(2)}, e_{-n}^{(1)}\right\rangle_{c} \\
\left\langle\widehat{T}_{n} \mathcal{Q}_{0} e_{-n}^{(1)}, e_{n}^{(2)}\right\rangle_{c} & \left\langle\widehat{T}_{n} \mathcal{Q}_{0} e_{n}^{(2)}, e_{n}^{(2)}\right\rangle_{c}
\end{array}\right)
$$

For any $\rho \geq 1$, denote by $\tilde{B}_{\rho}^{s+1}$ the closed ball of radius $\rho$ in $\tilde{H}_{c}^{s+1}$, centered at 0 ,

$$
\tilde{B}_{\rho}^{s+1}:=\left\{(q, \varphi) \in \tilde{H}_{c}^{s+1}: 1+R_{s}(q, \varphi) \leq \rho\right\}
$$

where we recall that $R_{s}(q, \varphi)=\|\varphi\|_{s}+\|\cosh (q / 2)\|_{s+1}+\|\sinh (q / 2)\|_{s+1}$.

Lemma 4.7. Let $s \geq 0, \rho \geq 1$, and $|n| \geq 2 C_{0} \rho^{4}$. Then the following holds:

(i) A complex number $\lambda \in \Pi_{n}$ is a periodic eigenvalue of $Q_{1} \partial_{x}+Q_{0}$ iff

$$
\operatorname{det} S_{n}(\lambda)=\left(\lambda-\pi n-a_{n}(\lambda)\right)^{2}-b_{n}^{+}(\lambda) b_{n}^{-}(\lambda)
$$

vanishes.

(ii) The functions $a_{n}^{ \pm}, b_{n}^{ \pm}$are analytic in $(\lambda,(q, \varphi))$ on $\Pi_{n} \times \tilde{B}_{\rho}^{s+1}$. Furthermore $a_{n}:=a_{n}^{+}$coincides with $a_{n}^{-}$and

$$
a_{n}(\lambda, q, \varphi)=\overline{a_{n}(\bar{\lambda}, \bar{q},-\bar{\varphi})}, \quad b_{n}^{-}(\lambda, q, \varphi)=\overline{b_{n}^{+}(\bar{\lambda}, \bar{q},-\bar{\varphi})} .
$$

Proof. (i) The statement follows from the definition of $S_{n}$ as mentioned in the discussion above.

(ii) By Lemma 4.5 (i), $T_{n}^{4}$ with $|n| \geq 2 C_{0} \rho^{4}$ is a $\frac{1}{2}$-contraction for any element in $\Pi_{n} \times \tilde{B}_{\rho}^{s+1}$. Hence $\left(I d-T_{n}^{4}\right)^{-1}$ can be expanded in its Neumann series, implying that $a_{n}(\lambda)$ and $b_{n}^{ \pm}(\lambda)$ can be written as series which converge normally and are analytic on $\Pi_{n} \times \tilde{B}_{\rho}^{s+1}$. Note that $\operatorname{det} Q_{0}(x)=(\operatorname{det} B(x))^{2}=\frac{1}{4}$. Hence $\mathcal{Q}_{0}(x)$ is invertible for any $x$ and $\widehat{T}_{n} \mathcal{Q}_{0}$ is invertible as an operator. By the definition of $T_{n}=\mathcal{Q}_{0} \mathcal{Q}(\lambda)^{-1} K_{n}$ it then follows that

$$
\begin{aligned}
\left(\mathcal{Q}_{0}^{-1}\left(I d-T_{n}\right)\right)^{*} & =\left(I d-\left(\mathcal{Q}(\lambda)^{-1} K_{n}\right)^{*} \mathcal{Q}_{0}^{*}\right)\left(\mathcal{Q}_{0}^{*}\right)^{-1} \\
& =\left(\mathcal{Q}_{0}^{*}\right)^{-1}\left(I d-\mathcal{Q}_{0}^{*}\left(\mathcal{Q}(\lambda)^{-1} K_{n}\right)^{*}\right) .
\end{aligned}
$$

Using that the adjoints of $\mathcal{Q}_{0}, \mathcal{Q}(\lambda)^{-1} K_{n}$ with respect to $\langle\cdot, \cdot\rangle_{c}$ are given by

$$
\mathcal{Q}_{0}(q, \varphi)^{*}=\mathcal{Q}_{0}(\bar{q},-\bar{\varphi}) \quad \text { and } \quad\left(\mathcal{Q}(\lambda)^{-1} K_{n}\right)^{*}=K_{n} \mathcal{Q}(\bar{\lambda})^{-1}=\mathcal{Q}(\bar{\lambda})^{-1} K_{n}
$$

one has

$$
\left(\mathcal{Q}_{0}^{-1}(q, \varphi)\left(I d-T_{n}(\lambda, q, \varphi)\right)\right)^{*}=\left(\mathcal{Q}_{0}^{-1}(\bar{q},-\bar{\varphi})\left(I d-T_{n}(\bar{\lambda}, \bar{q},-\bar{\varphi})\right)\right) .
$$


Taking the inverse of both sides of the latter identity one gets

$$
\left(\widehat{T}_{n}(\lambda, q, \varphi) \mathcal{Q}_{0}(q, \varphi)\right)^{*}=\widehat{T}_{n}(\bar{\lambda}, \bar{q},-\bar{\varphi}) \mathcal{Q}_{0}(\bar{q},-\bar{\varphi})
$$

Hence $b_{n}^{+}(\lambda, q, \varphi)=\overline{b_{n}^{-}(\bar{\lambda}, \bar{q},-\bar{\varphi})}$ and $a_{n}^{ \pm}(\lambda, q, \varphi)=\overline{a_{n}^{ \pm}(\bar{\lambda}, \bar{q},-\bar{\varphi})}$. It remains to prove that $a_{n}^{+}=a_{n}^{-}$. For a given linear operator $B$ acting on a $\mathbb{C}$-vector space, denote by $\bar{B}$ its complex conjugate defined by $\bar{B} u:=\bar{B} \bar{u}$. Furthermore, note that

$$
\left(\begin{array}{ll}
Z & \\
& Z
\end{array}\right) e_{n}^{(1)}=e_{n}^{(2)} \text { and } \quad\left(\begin{array}{ll}
Z & \\
& Z
\end{array}\right) e_{n}^{(2)}=e_{n}^{(1)} .
$$

Using that $\overline{e_{-n}^{(2)}}=e_{n}^{(2)}$ and $\langle a, b\rangle_{c}=\langle\bar{b}, \bar{a}\rangle_{c}$ one then gets

$$
\begin{aligned}
& a_{n}^{+}=\left\langle\widehat{T}_{n} \mathcal{Q}_{0} e_{-n}^{(1)}, e_{-n}^{(1)}\right\rangle_{c}=\left\langle\widehat{T}_{n} \mathcal{Q}_{0}\left(\begin{array}{ll}
Z & \\
& Z
\end{array}\right) e_{-n}^{(2)},\left(\begin{array}{ll}
Z & \\
& Z
\end{array}\right) e_{-n}^{(2)}\right\rangle_{c} \\
& =\left\langle e_{-n}^{(2)},\left(\begin{array}{ll}
Z & \\
& Z
\end{array}\right)\left(\widehat{T}_{n} \mathcal{Q}_{0}\right)^{*}\left(\begin{array}{ll}
Z & \\
& Z
\end{array}\right) e_{-n}^{(2)}\right\rangle_{c} \\
& =\left\langle\left(\begin{array}{ll}
Z & \\
& Z
\end{array}\right) \overline{\left(\widehat{T}_{n} \mathcal{Q}_{0}\right)^{*}}\left(\begin{array}{ll}
Z & \\
& Z
\end{array}\right) e_{n}^{(2)}, e_{n}^{(2)}\right\rangle_{c} .
\end{aligned}
$$

It remains to compute

$$
\left(\begin{array}{ll}
Z & \\
& Z
\end{array}\right) \overline{\left(\widehat{T}_{n} \mathcal{Q}_{0}\right)^{*}}\left(\begin{array}{ll}
Z & \\
& Z
\end{array}\right)
$$

A straightforward computation yields

$$
\begin{aligned}
\mathcal{Q}_{0}(q, \varphi)^{*} & =\left(\begin{array}{ll}
Z & \\
& Z
\end{array}\right) \overline{\mathcal{Q}_{0}(q, \varphi)}\left(\begin{array}{ll}
Z & \\
& Z
\end{array}\right), \\
\left(\mathcal{Q}(\lambda)^{-1} K_{n}\right)^{*} & =\left(\begin{array}{ll}
Z & \\
& Z
\end{array}\right) \overline{\mathcal{Q}(\lambda)^{-1} K_{n}}\left(\begin{array}{ll}
Z & \\
& Z
\end{array}\right) .
\end{aligned}
$$

Hence the adjoint of $\widehat{T}_{n} \mathcal{Q}_{0}=\left(I d-\mathcal{Q}_{0} \mathcal{Q}(\lambda)^{-1} K_{n}\right)^{-1} \mathcal{Q}_{0}$ is given by

$$
\left(\widehat{T}_{n} \mathcal{Q}_{0}\right)^{*}=\left(\begin{array}{ll}
Z & \\
& Z
\end{array}\right) \overline{\left(\widehat{T}_{n} \mathcal{Q}_{0}\right)}\left(\begin{array}{ll}
Z & \\
& Z
\end{array}\right) .
$$

This implies that $a_{n}^{+}(\lambda)=a_{n}^{-}(\lambda)$.

For a function $f: U \rightarrow \mathbb{C}$, defined on a domain $U \subset X$ of a $\mathbb{C}$-Banach space $(X,\|\cdot\|)$, denote by $|f|_{U}$ its sup norm,

$$
|f|_{U}:=\sup _{\lambda \in U}\|f(\lambda)\| .
$$


Lemma 4.8. Let $(q, \varphi) \in \tilde{H}_{c}^{1}$ and $|n| \geq 2 C_{0} R_{0}^{4}(q, \varphi)$. Then

$$
\left|a_{n}\right|_{\Pi_{n}} \leq \frac{1}{|n|}\left(1+R_{0}(q, \varphi)\right)^{4} R_{0}^{2}(q, \varphi)+\left(1+R_{0}(q, \varphi)\right)^{4}\|\varphi\|_{L^{2}} R_{0 ;|n|}(\varphi) .
$$

Furthermore, if in addition $\varphi \in H_{\mathbb{C}}^{s}$ for some $s \geq 0$, one has $R_{0 ;|n|}(\varphi) \leq \frac{1}{\langle n\rangle^{s}}\|\varphi\|_{s}$ and hence

$$
\left|a_{n}\right|_{\Pi_{n}} \leq 2\left(1+R_{0}(q, \varphi)\right)^{4}\left(\frac{R_{0}^{2}(q, \varphi)}{|n|}+\frac{\|\varphi\|_{L^{2}}\|\varphi\|_{s}}{\langle n\rangle^{s}}\right) .
$$

Proof. Since $\widehat{T}_{n}=I d+\widehat{T}_{n} T_{n}=I d+T_{n}+\widehat{T}_{n} T_{n}^{2}$ and

$$
\mathcal{Q}_{0} e_{-n}^{(1)}=\frac{1}{4}\left(0,-\varphi e_{-n}, \cosh (q / 2) e_{-n},-\sinh (q / 2) e_{-n}\right),
$$

one has $\left\langle\mathcal{Q}_{0} e_{-n}^{(1)}, e_{-n}^{(1)}\right\rangle_{c}=0$. Using that $\widehat{T}_{n}=I d+T_{n}+\widehat{T}_{n} T_{n}^{2}$ we split $a_{n}$ into a sum, $a_{n}=\Sigma_{1}+\Sigma_{2}$, where

$$
\Sigma_{1}:=\left\langle T_{n} \mathcal{Q}_{0} e_{-n}^{(1)}, e_{-n}^{(1)}\right\rangle_{c}, \quad \Sigma_{2}:=\left\langle\widehat{T}_{n} T_{n}^{2} \mathcal{Q}_{0} e_{-n}^{(1)}, e_{-n}^{(1)}\right\rangle_{c} .
$$

Substitute

$$
\varphi=\sum_{m \in \mathbb{Z}} \varphi_{m} e_{m} \quad \text { where } \quad \varphi_{m} \equiv \widehat{\varphi}(m)=\int_{0}^{1} \varphi(x) e^{-\mathrm{i} m \pi x} \mathrm{~d} x
$$

into $T_{n} \mathcal{Q}_{0} e_{-n}^{(1)}=\mathcal{Q}_{0} \mathcal{Q}(\lambda)^{-1} K_{n} \mathcal{Q}_{0} e_{-n}^{(1)}$ to obtain

$$
\begin{aligned}
T_{n} \mathcal{Q}_{0} e_{-n}^{(1)} & =\mathcal{Q}_{0} \mathcal{Q}(\lambda)^{-1} K_{n} \frac{1}{4}\left(\begin{array}{c}
0 \\
-\varphi e_{-n} \\
\cosh (q / 2) e_{-n} \\
-\sinh (q / 2) e_{-n}
\end{array}\right) \\
& =\mathcal{Q}_{0} \mathcal{Q}(\lambda)^{-1} \frac{1}{4}\left(\begin{array}{c}
0 \\
-\sum_{m \neq n} \varphi_{m+n} e_{m} \\
\cosh (q / 2) e_{-n} \\
-\sinh (q / 2) e_{-n}
\end{array}\right)
\end{aligned}
$$

and by (4.9)

$$
\mathcal{Q}(\lambda)^{-1} \frac{1}{4}\left(\begin{array}{c}
0 \\
-\sum_{m \neq n} \varphi_{m+n} e_{m} \\
\cosh (q / 2) e_{-n} \\
-\sinh (q / 2) e_{-n}
\end{array}\right)=\frac{1}{4}\left(\begin{array}{c}
0 \\
-\sum_{m \neq n} \varphi_{m+n} \frac{e_{m}}{\lambda-n \pi} \\
\frac{1}{\lambda} \cosh (q / 2) e_{-n} \\
-\frac{1}{\lambda} \sinh (q / 2) e_{-n}
\end{array}\right) .
$$

Using trigonometric identities, one then concludes

$$
T_{n} \mathcal{Q}_{0} e_{-n}^{(1)}=\frac{1}{16 \lambda}\left(\begin{array}{c}
\cosh (q) e_{-n} \\
-\sinh (q) e_{-n} \\
0 \\
0
\end{array}\right)-\frac{1}{16} \sum_{m \neq n} \frac{\varphi_{m+n}}{\lambda-m \pi} e_{m}\left(\begin{array}{c}
\varphi \\
0 \\
-\sinh (q / 2) \\
\cosh (q / 2)
\end{array}\right)
$$


Hence by (4.19), for any $\lambda \in \Pi_{n}$,

$$
\begin{aligned}
\left|\Sigma_{1}\right| & =\left|\left\langle T_{n} \mathcal{Q}_{0} e_{-n}^{(1)}, e_{-n}^{(1)}\right\rangle_{c}\right| \\
& \leq \frac{1}{16|\lambda|}|\widehat{\cosh (q)}(0)|+\frac{1}{16} \sum_{m \neq n} \frac{\left|\varphi_{m+n}\right|}{|n-m|}\left|\varphi_{-(m+n)}\right| .
\end{aligned}
$$

Since $\cosh (q)=\cosh ^{2}(q / 2)+\sinh ^{2}(q / 2)$ one has by Lemma 4.1

$$
\|\cosh (q)\|_{L^{2}} \leq 2\|\cosh (q / 2)\|_{1}^{2}+2\|\sinh (q / 2)\|_{1}^{2}
$$

and hence

$$
|\widehat{\cosh (q)}(0)| \leq\|\cosh (q)\|_{L^{2}} \leq 2\|\cosh (q / 2)\|_{1}^{2}+2\|\sinh (q / 2)\|_{1}^{2} .
$$

For the second term in (4.50), we split the sum into two parts, $|m-n|>|n|$ and $1 \leq|m-n| \leq|n|$ to get

$$
\frac{1}{16} \sum_{m \neq n} \frac{\left|\varphi_{m+n}\right|}{|n-m|}\left|\varphi_{-(m+n)}\right| \leq \frac{1}{16|n|}\|\varphi\|_{L^{2}}^{2}+\frac{1}{16} \sum_{1 \leq|m-n| \leq|n|}\left|\varphi_{m+n} \| \varphi_{-(m+n)}\right| .
$$

Using that for $|m-n| \leq|n|$ one has $|m+n|=|2 n+m-n| \geq 2|n|-|n|=|n|$ and hence

$$
\sum_{1 \leq|m-n| \leq|n|}\left|\varphi_{m+n}\right|\left|\varphi_{-(m+n)}\right| \leq\|\varphi\|_{L^{2}} R_{0 ;|n|}(\varphi) .
$$

Altogether we thus have shown that

$$
\left|\Sigma_{1}\right| \leq \frac{R_{0}^{2}}{8|n|}+\frac{1}{16}\|\varphi\|_{L^{2}} R_{0 ;|n|}(\varphi) .
$$

Towards $\Sigma_{2}$ note that for any vector valued $L^{2}$-function $f$ and $\left|\left\langle f, e_{-n}^{(i)}\right\rangle_{c}\right| \leq$ $\|f\|_{L^{2}}, 1 \leq i \leq 4$. Hence for $f=\widehat{T}_{n} T_{n}^{2} \mathcal{Q}_{0} e_{-n}^{(1)}$,

$$
\left|\Sigma_{2}\right|=\left|\left\langle\widehat{T}_{n} T_{n}^{2} \mathcal{Q}_{0} e_{-n}^{(1)}, e_{-n}^{(1)}\right\rangle_{c}\right| \leq\left\|\widehat{T}_{n} T_{n}^{2} \mathcal{Q}_{0} e_{-n}^{(1)}\right\|_{L^{2}} .
$$

Hence by (4.41)

$$
\left|\Sigma_{2}\right| \leq\left\|\widehat{T}_{n} T_{n}^{2} \mathcal{Q}_{0} e_{-n}^{(1)}\right\|_{L^{2}} \leq 2\left(1+R_{0}\right)^{3}\left\|T_{n}^{2} \mathcal{Q}_{0} e_{-n}^{(1)}\right\|_{L^{2}} .
$$

Furthermore, by (4.49),

$$
\begin{aligned}
T_{n}^{2} \mathcal{Q}_{0} e_{-n}^{(1)}=\frac{1}{16 \lambda} T_{n}\left(\begin{array}{c}
\cosh (q) e_{-n} \\
-\sinh (q) e_{-n} \\
0 \\
0
\end{array}\right) & -\frac{1}{64 \lambda} \sum_{m \neq n} \frac{\varphi_{m+n}}{\lambda-m \pi} e_{m}\left(\begin{array}{c}
-\sinh (q) \\
\cosh (q) \\
0 \\
0
\end{array}\right) \\
& -T_{n} \frac{1}{16} \sum_{m \neq n} \frac{\varphi_{m+n}}{\lambda-m \pi} \varphi e_{m}^{(1)}
\end{aligned}
$$


We will now estimate the three terms in the latter expression separately. One easily checks, using Lemma 4.2, that for any $\lambda \in \Pi_{n}$

$$
\left\|\frac{1}{16 \lambda} T_{n}\left(\begin{array}{c}
\cosh (q) e_{-n} \\
-\sinh (q) e_{-n} \\
0 \\
0
\end{array}\right)\right\|_{L^{2}} \leq \frac{1}{16|n|} R_{0}\left(\|\cosh (q)\|_{L^{2}}+\|\sinh (q)\|_{L^{2}}\right) .
$$

For the second term in (4.53) one has for any $\lambda \in \Pi_{n}$

$$
\left\|\frac{1}{64 \lambda} \sum_{m \neq n} \frac{\varphi_{m+n}}{\lambda-m \pi} e_{m}\left(\begin{array}{c}
-\sinh (q) \\
\cosh (q) \\
0 \\
0
\end{array}\right)\right\|_{L^{2}} \leq \frac{1}{64|n|}\left(\|\sinh (q)\|_{L^{2}}+\|\cosh (q)\|_{L^{2}}\right)\|\varphi\|_{L^{2}} .
$$

Finally, for the last term in (4.53) one has by Lemma 4.2

$$
\left\|T_{n} \frac{1}{16} \sum_{m \neq n} \frac{\varphi_{m+n}}{\lambda-m \pi} \varphi e_{m}^{(1)}\right\|_{L^{2}} \leq R_{0} \frac{1}{16}\|\varphi\|_{L^{2}}\left(\sum_{m \neq n} \frac{\left|\varphi_{m+n}\right|^{2}}{|n-m|^{2}}\right)^{1 / 2},
$$

where by arguing as above,

$$
\left(\sum_{m \neq n} \frac{\left|\varphi_{m+n}\right|^{2}}{|n-m|^{2}}\right)^{1 / 2} \leq \frac{1}{|n|}\|\varphi\|_{L^{2}}+R_{0 ;|n|}(\varphi) .
$$

Altogether we have proved

$$
\begin{aligned}
\left\|T_{n}^{2} \mathcal{Q}_{0} e_{-n}^{(1)}\right\|_{L^{2}} \leq & \frac{R_{0}}{4|n|}\left(\|\varphi\|_{L^{2}}^{2}+\frac{1}{2}\left(\|\cosh (q)\|_{L^{2}}+\|\sinh (q)\|_{L^{2}}\right)\right) \\
& +\frac{R_{0}}{16}\|\varphi\|_{L^{2}} R_{0 ;|n|}(\varphi) .
\end{aligned}
$$

By Lemma $4.1\|\sinh (q)\|_{L^{2}} \leq 4\|\cosh (q / 2)\|_{1}\|\sinh (q / 2)\|_{1}$ and with (4.51) one obtains

$$
\begin{aligned}
\|\varphi\|_{L^{2}}^{2}+\frac{1}{2}\left(\|\cosh (q)\|_{L^{2}}+\|\right. & \left.\sinh (q) \|_{L^{2}}\right) \\
& \leq\|\varphi\|_{L^{2}}^{2}+\left(\|\cosh (q / 2)\|_{1}+\|\sinh (q / 2)\|_{1}\right)^{2} \leq R_{0}^{2} .
\end{aligned}
$$

Hence by (4.52),

$$
\left|\Sigma_{2}\right| \leq \frac{1}{2|n|}\left(1+R_{0}\right)^{3} R_{0}^{3}+\left(1+R_{0}\right)^{3} R_{0}\|\varphi\|_{L^{2}} R_{0 ;|n|}(\varphi) .
$$

Combining the estimates for $\Sigma_{1}$ and $\Sigma_{2}$ yields (4.46). The estimate (4.47) immediately follows from (4.46).

Next we estimate $b_{n}^{+}(\lambda), b_{n}^{-}(\lambda)$, introduced in (4.43). 
Lemma 4.9. Let $s \geq 0,(q, \varphi) \in \tilde{H}_{c}^{s+1}$ and $\lambda \in \Pi_{n}$ with $|n| \geq 2 C_{0} R_{s}^{4}$. Then the following holds:

$$
\begin{aligned}
& \langle n\rangle^{s}\left|b_{n}^{ \pm}(\lambda) \mp \frac{1}{4} \widehat{\varphi}(\mp 2 n)\right|_{\Pi_{n}} \leq \frac{1}{2|n|}\left(1+R_{s}(q, \varphi)\right)^{6}, \\
& \left|b_{n}^{+} b_{n}^{-}\right|_{\Pi_{n}} \leq \frac{|\widehat{\varphi}(-2 n)|^{2}+|\widehat{\varphi}(2 n)|^{2}}{16}+\frac{1}{2 n^{2}}\left(1+R_{0}(q, \varphi)\right)^{12} \\
& \langle n\rangle^{2 s}\left|b_{n}^{+} b_{n}^{-}\right|_{\Pi_{n}} \leq \frac{1}{16}\|\varphi\|_{s}^{2}+\frac{1}{2 n^{2}}\left(1+R_{s}(q, \varphi)\right)^{12} .
\end{aligned}
$$

Furthermore

$$
\left(\sum_{n \geq 2 C_{0}\left(1+R_{s}\right)^{4}+1}\langle n\rangle^{2 s} 6\left|b_{n}^{+} b_{n}^{-}\right|_{\Pi_{n}}\right)^{1 / 2} \leq\|\varphi\|_{s}+2\left(1+R_{s}(q, \varphi)\right)^{4} .
$$

Proof. We begin by proving the estimate (4.54) for $b_{n}^{-}(\lambda)$ for $n$ with $|n| \geq$ $2 C_{0} R_{s}^{4}$. By the definition (4.43), $b_{n}^{-}(\lambda)=\left\langle\widehat{T}_{n} \mathcal{Q}_{0} e_{-n}^{(1)}, e_{n}^{(2)}\right\rangle_{c}$. Arguing as in the proof of Lemma 4.8, one gets for any $\lambda \in \Pi_{n}$

$$
b_{n}^{-}(\lambda)=\left\langle\mathcal{Q}_{0} e_{-n}^{(1)}, e_{n}^{(2)}\right\rangle_{c}+\left\langle T_{n} \mathcal{Q}_{0} e_{-n}^{(1)}, e_{n}^{(2)}\right\rangle_{c}+\left\langle\widehat{T}_{n} T_{n}^{2} \mathcal{Q}_{0} e_{-n}^{(1)}, e_{n}^{(2)}\right\rangle_{c} .
$$

By (4.48), one has

$$
\left\langle\mathcal{Q}_{0} e_{-n}^{(1)}, e_{n}^{(2)}\right\rangle_{c}=-\frac{1}{4} \int_{0}^{1} \varphi e_{-n} e_{-n} \mathrm{~d} x=-\frac{1}{4} \widehat{\varphi}(2 n) .
$$

By (4.49) and (4.6), for any $\lambda \in \Pi_{n}$

$$
\begin{aligned}
\left|\left\langle T_{n} \mathcal{Q}_{0} e_{-n}^{(1)}, e_{n}^{(2)}\right\rangle_{c}\right| & =\frac{1}{16|\lambda|}|\widehat{\sinh (q)}(2 n)| \leq \frac{1}{16|\lambda|\langle 2 n\rangle^{s}}\|\sinh (q)\|_{s} \\
& \leq \frac{1}{4|n|\langle 2 n\rangle^{s}}\|\sinh (q / 2)\|_{s+1}\|\cosh (q / 2)\|_{s+1} .
\end{aligned}
$$

By (4.41),

$$
\langle n\rangle^{s}\left|\left\langle\widehat{T}_{n} T_{n}^{2} \mathcal{Q}_{0} e_{-n}^{(1)}, e_{n}^{(2)}\right\rangle_{c}\right| \leq\left\|\widehat{T}_{n} T_{n}^{2} \mathcal{Q}_{0} e_{-n}^{(1)}\right\|_{s ; n} \leq 2\left(1+R_{s}\right)^{3}\left\|T_{n}^{2} \mathcal{Q}_{0} e_{-n}^{(1)}\right\|_{s ; n} .
$$

Since by (4.48), the first entry of $\mathcal{Q}_{0} e_{-n}^{(1)}$ vanishes one can apply Lemma 4.5 (iii) to obtain $\left\|T_{n}^{2} \mathcal{Q}_{0} e_{-n}^{(1)}\right\|_{s ; n} \leq \frac{1}{|n|} R_{s}^{2}\left\|\mathcal{Q}_{0} e_{-n}^{(1)}\right\|_{s ; n}$. Similarly, by the formula (4.48) for $\mathcal{Q}_{0} e_{-n}^{(1)}$ one has $\left\|\mathcal{Q}_{0} e_{-n}^{(1)}\right\|_{s ; n} \leq \frac{1}{4} R_{s}$ and the claimed estimate of $b_{n}^{-}(\lambda)$ of $(4.54)$ follows. The estimate for $b_{n}^{+}(\lambda)$ is proved in a similar fashion. To prove (4.55) and (4.56) use that $|a b| \leq \frac{1}{2}\left(|a|^{2}+|b|^{2}\right)$ to obtain for $\lambda \in \Pi_{n}$

$$
\left|b_{n}^{+} b_{n}^{-}\right| \leq\left(\left|b_{n}^{+}-\frac{1}{4} \widehat{\varphi}(-2 n)\right|+\left|\frac{1}{4} \widehat{\varphi}(-2 n)\right|\right)\left(\left|b_{n}^{-}+\frac{1}{4} \widehat{\varphi}(2 n)\right|+\left|\frac{1}{4} \widehat{\varphi}(2 n)\right|\right)
$$




$$
\leq\left|b_{n}^{+}-\frac{1}{4} \widehat{\varphi}(-2 n)\right|^{2}+\left|\frac{1}{4} \widehat{\varphi}(-2 n)\right|^{2}+\left|b_{n}^{-}+\frac{1}{4} \widehat{\varphi}(2 n)\right|^{2}+\left|\frac{1}{4} \widehat{\varphi}(2 n)\right|^{2} .
$$

Hence by (4.54), for any $\lambda \in \Pi_{n}$,

$$
\left|b_{n}^{+} b_{n}^{-}\right| \leq \frac{1}{16}\left(|\widehat{\varphi}(-2 n)|^{2}+|\widehat{\varphi}(2 n)|^{2}\right)+\frac{1}{2 n^{2}\langle n\rangle^{2 s}}\left(1+R_{s}\right)^{12} .
$$

For $s=0$, this yields (4.55) and for $s \geq 0$ arbitrary (4.56). Finally since

$$
\sum_{n \geq 2 C_{0}\left(1+R_{s}\right)^{4}+1}\langle n\rangle^{2 s}|\widehat{\varphi}(-2 n)|^{2}+\langle n\rangle^{2 s}|\widehat{\varphi}(2 n)|^{2} \leq\|\varphi\|_{s}^{2}
$$

and since $C_{0} \geq 1$ and therefore

$$
\sum_{n \geq 2 C_{0}\left(1+R_{s}\right)^{4}+1} \frac{1}{n^{2}} \leq \int_{2 C_{0}\left(1+R_{s}\right)^{4}}^{\infty} \frac{1}{x^{2}} \mathrm{~d} x=\frac{1}{2 C_{0}\left(1+R_{s}\right)^{4}}
$$

(4.57) follows from (4.58).

We now prove the following stronger version of Theorem 1.1.

Theorem 4.10. Let $s \geq 0$ and $(q, p) \in H_{c}^{s+1}$. Then there exists $N_{1} \geq 2 C_{0}(1+$ $\left.R_{s}\right)^{4}+1$ so that for any $|n| \geq N_{1}$, det $S_{n}=\left(\lambda-\pi n-a_{n}(\lambda)\right)^{2}-b_{n}^{+}(\lambda) b_{n}^{-}(\lambda)$ has, when counted with multiplicities, exactly two roots $\lambda_{n}^{ \pm}$in $\Pi_{n}$. They are contained in $D_{n} \subset \Pi_{n}$. Furthermore, $\gamma_{n}=\lambda_{n}^{+}-\lambda_{n}^{-}$satisfy

$$
\left|\gamma_{n}\right|^{2} \leq 6\left|b_{n}^{+} b_{n}^{-}\right|_{\Pi_{n}}, \quad n \geq N_{1}
$$

and

$$
\left(\sum_{n \geq N_{1}}\langle n\rangle^{2 s}\left|\gamma_{n}(q, p)\right|^{2}\right)^{1 / 2} \leq\|\varphi(q, p)\|_{s}+2\left(1+R_{s}(q, p)\right)^{4}
$$

where by a slight abuse of terminology,

$$
R_{s}(q, p) \equiv R_{s}(q, \varphi(q, p))=\|\varphi\|_{s}+\|\sinh (q / 2)\|_{s+1}+\|\cosh (q / 2)\|_{s+1} .
$$

Remark 4.11. Recall that by the reciprocity law, for any $n \geq 0$,

$$
\frac{1}{16 \lambda_{-n}^{-}(q, p)}-\frac{1}{16 \lambda_{-n}^{+}(q, p)}=\lambda_{n}^{+}(-q, p)-\lambda_{n}^{-}(-q, p)=\gamma_{n}(-q, p) .
$$

Hence (4.59) applied to $(-q, p)$ leads to the estimate

$$
\left|\frac{1}{16 \lambda_{-n}^{-}(q, p)}-\frac{1}{16 \lambda_{-n}^{+}(q, p)}\right|^{2} \leq 6\left|b_{n}^{+} b_{n}^{-}\right|_{\Pi_{n},-q, p}, \quad n \geq N_{1} .
$$


Proof. By assumption $(q, \varphi) \in \tilde{H}^{s+1}$. According to Lemma 4.8 and 4.9, there exists $N_{1} \geq 2 C_{0}\left(1+R_{0}\right)^{4}+1$ so that for any $|n| \geq N_{1},\left|a_{n}\right|_{\Pi_{n}},\left|b_{n}^{+} b_{n}^{-}\right|_{\Pi_{n}} \leq \frac{\pi}{48}$. Hence, for any $|n| \geq N_{1}$ and $\lambda \in \Pi_{n}$

$$
\left|\operatorname{det} S_{n}(\lambda)-\left(\lambda-n \pi-a_{n}(\lambda)\right)^{2}\right| \leq\left|b_{n}^{+}(\lambda) b_{n}^{-}(\lambda)\right|^{2} \leq\left(\frac{\pi}{48}\right)^{2}
$$

and

$$
\inf _{\lambda \in \partial D_{n}}\left|\lambda-n \pi-a_{n}(\lambda)\right|^{2} \geq\left|\frac{\pi}{3}-\sup _{\lambda \in \partial D_{n}}\right| a_{n}(\lambda)||^{2}>\left(\frac{\pi}{48}\right)^{2} .
$$

As det $S_{n}(\lambda)$ and $\left(\lambda-n \pi-a_{n}(\lambda)\right)^{2}$ are both analytic on $\Pi_{n}$, by Rouché's Theorem, they have the same number of roots in $D_{n}$ when counted with multiplicities. By the same argument, one shows that $\left(\lambda-n \pi-a_{n}(\lambda)\right)^{2}$ and $(\lambda-n \pi)^{2}$ have the same number of roots in $D_{n}$ when counted with multiplicities. Hence $\operatorname{det} S_{n}(\lambda)$ has two roots in $D_{n}$. By choosing $N_{1}$ larger than the integer $N$ in Lemma 3.11 (Counting Lemma), it follows that these two roots are precisely the periodic eigenvalues $\lambda_{n}^{+}$ and $\lambda_{n}^{-}$.

To prove the claimed estimate for the gaps $\gamma_{n}=\lambda_{n}^{+}-\lambda_{n}^{-}$, we write

$$
\operatorname{det} S_{n}=\left(\lambda-\pi n-a_{n}\right)^{2}-b_{n}^{+} b_{n}^{-}=g_{+} g_{-},
$$

where

$$
g_{ \pm}=\lambda-n \pi-a_{n} \pm \sigma_{n}, \quad \sigma_{n}=\sqrt{b_{n}^{+} b_{n}^{-}}
$$

and the choice of the branch of the root does not matter. Each root $\xi_{n} \in D_{n}$ of $\operatorname{det} S_{n}$ is either a root of $g_{+}$or $g_{-}$and hence is of the form $\xi_{n}=n \pi+a_{n}\left(\xi_{n}\right) \pm$ $\sigma\left(\xi_{n}\right)$. It then follows that

$\left|\lambda_{n}^{+}-\lambda_{n}^{-}\right| \leq\left|a_{n}\left(\lambda_{n}^{+}\right)-a_{n}\left(\lambda_{n}^{-}\right)\right|+\left|\sigma_{n}\left(\lambda_{n}^{+}\right)\right|+\left|\sigma_{n}\left(\lambda_{n}^{-}\right)\right| \leq\left|\partial_{\lambda} a_{n}\right|_{D_{n}}\left|\lambda_{n}^{+}-\lambda_{n}^{-}\right|+2\left|\sigma_{n}\right|_{\Pi_{n}}$.

Since $\operatorname{dist}\left(D_{n}, \partial \Pi_{n}\right) \geq \pi / 6$, we obtain from Cauchy's estimate $\left|\partial_{\lambda} a_{n}\right|_{D_{n}} \leq$ $\frac{\left|a_{n}\right|_{\Pi_{n}}}{\pi / 6} \leq \frac{1}{8}$, which implies that $\frac{7}{8}\left|\lambda_{n}^{+}-\lambda_{n}^{-}\right| \leq 2\left|\sqrt{b_{n}^{+} b_{n}^{-}}\right|_{\Pi_{n}}$ and therefore $\mid \lambda_{n}^{+}-$ $\left.\lambda_{n}^{-}\right|^{2} \leq 6\left|b_{n}^{+} b_{n}^{-}\right|_{\Pi_{n}}$. By Lemma 4.9 one then gets that

$$
\left(\sum_{n \geq N_{1}}\langle n\rangle^{2 s}\left|\gamma_{n}\right|^{2}\right)^{1 / 2} \leq\|\varphi\|_{s}+2\left(1+R_{s}\right)^{4} .
$$

Together with Lemma 2.14 (reciprocity in $\lambda$ ), this yields (4.59) and (4.60).

Remark 4.12. Assume that $(q, p) \in H_{c}^{1}$ is real valued. Then $-\bar{\varphi}=\varphi$ and hence by Lemma 4.7

$$
b_{n}^{-}(\lambda, q, \varphi)=\overline{b_{n}^{+}(\lambda, q, \varphi)}, \quad a_{n}(\lambda, q, \varphi) \in \mathbb{R}, \quad \forall \lambda \in \mathbb{R}^{*} .
$$

Furthermore, by Lemma $4.9(s=0)$,

$$
\left|b_{n}^{+}\left(\lambda_{n}^{ \pm}\right)-\frac{1}{4} \widehat{\varphi}(-2 n)\right|=O\left(\frac{1}{n}\right)
$$


and hence

$$
\sigma_{n}\left(\lambda_{n}^{ \pm}\right)=\left|b_{n}^{+}\left(\lambda_{n}^{ \pm}\right)\right|=\frac{1}{4}|\widehat{\varphi}(-2 n)|+O\left(\frac{1}{n}\right) .
$$

On the other hand, by definition

$$
a_{n}(\lambda)=\left\langle\left(I d-T_{n}(\lambda)\right)^{-1} \mathcal{Q}_{0} e_{-n}^{(1)}, e_{-n}^{(1)}\right\rangle_{c} .
$$

Expanding $\left(I d-T_{n}(\lambda)\right)^{-1}$ in the form

$$
\left(I d-T_{n}(\lambda)\right)^{-1}=I d+\sum_{k=1}^{3} T_{n}(\lambda)^{k}+T_{n}(\lambda)^{4}\left(I d-T_{n}(\lambda)\right)^{-1}
$$

(4.5) and (4.48) yield

$$
a_{n}\left(\lambda_{n}^{ \pm}\right)=\sum_{k=1}^{3}\left\langle T_{n}\left(\lambda_{n}^{ \pm}\right)^{k} \mathcal{Q}_{0} e_{-n}^{(1)}, e_{-n}^{(1)}\right\rangle_{c}+O\left(\frac{1}{n}\right) .
$$

By (4.61), (4.62), there exists $\rho_{n}^{ \pm} \in\{1,-1\}$ so that $\lambda_{n}^{ \pm}=n \pi+a_{n}\left(\lambda_{n}^{ \pm}\right)+\rho_{n}^{ \pm} \sigma_{n}\left(\lambda_{n}^{ \pm}\right)$. It implies that

$$
\lambda_{n}^{+}-\lambda_{n}^{-}=\sum_{k=1}^{3}\left\langle\left(T_{n}\left(\lambda_{n}^{+}\right)^{k}-T_{n}\left(\lambda_{n}^{-}\right)^{k}\right) \mathcal{Q}_{0} e_{-n}^{(1)}, e_{-n}^{(1)}\right\rangle_{c}+\left(\rho_{n}^{+}-\rho_{n}^{-}\right) \frac{1}{4}|\widehat{\varphi}(-2 n)|+O\left(\frac{1}{n}\right) .
$$

For $|n|$ sufficiently large the $2 \times 2$ matrix $S_{n}$ contains all the information about the $n$th periodic eigenvalues of a potential. In order to study their asymptotics for $|n| \rightarrow \infty$ in terms of the regularity of the potential, we analyze $S_{n}(\lambda), \lambda \in \Pi_{n}$, further. We will prove that the diagonal of $S_{n}(\lambda)$ vanishes at a unique point $\lambda=$ $\sigma_{n}(q, \varphi)$. These values will be used to locally define a real analytic perturbation of the Fourier transform which allows to characterize the regularity of potentials mentioned above. First we need to establish some auxiliary results.

Lemma 4.13. Let $s>0$ and $\rho>0$. Then for any $(q, \varphi) \in \tilde{B}_{\rho}^{s+1}$ and $|n| \geq$ $\max \left(2 C_{0} \rho^{4}, \sqrt[s]{96 \rho^{6}}, 96 \rho^{6}\right)$, there is a unique analytic function $\sigma_{n}: \tilde{B}_{\rho}^{s+1} \rightarrow \mathbb{C}$ such that

(i) $\sigma_{n}(q, \varphi)=n \pi+a_{n}\left(\sigma_{n}(q, \varphi),(q, \varphi)\right),(q, \varphi) \in \tilde{B}_{\rho}^{s+1}$,

(ii) $\sup _{(q, \varphi) \in \tilde{B}_{\rho}^{s+1}}\left|\sigma_{n}(q, \varphi)-n \pi\right| \leq \frac{\pi}{48}$,

(iii) $\sigma_{n}(q, \varphi) \in \mathbb{R}$ for any real valued $(q, \varphi) \in \tilde{B}_{\rho}^{s+1}$.

Proof. For any given $|n| \geq \max \left(2 C_{0} \rho^{4}, \sqrt[s]{48 \rho^{6}}, 96 \rho^{6}\right)$, consider the map $T$ with domain of definition $E:=\left\{\sigma: \tilde{B}_{\rho}^{s+1} \rightarrow D_{n}^{\prime}: \sigma\right.$ real analytic $\}$ and $D_{n}^{\prime}=$ $\{\lambda \in \mathbb{C}:|\lambda-n \pi| \leq \pi / 48\} \subset D_{n}$, defined by

$$
T \sigma:=n \pi+a_{n}(\sigma(\cdot), \cdot) .
$$


The set $E$ is obviously not empty since the constant function $\sigma \equiv n \pi$ is in $E$. Note that by the definition of $\tilde{B}_{\rho}^{s+1}$, the assumed lower bound for $|n|$, and Lemma 4.8

$$
\left|a_{n}\right|_{\Pi_{n} \times \tilde{B}_{\rho}^{s+1}} \leq 2\left(1+R_{0}\right)^{4}\left(\frac{R_{0}^{2}}{|n|}+\frac{\|\varphi\|_{L^{2}}\|\varphi\|_{s}}{\langle n\rangle^{s}}\right) \leq 2 \rho^{4}\left(\frac{\rho^{2}}{96 \rho^{6}}+\frac{\rho^{2}}{96 \rho^{6}}\right) \leq \frac{\pi}{48}
$$

implying that $T$ maps $E$ into $E$. Endow $E$ with the metric $d\left(\sigma_{1}, \sigma_{2}\right)=\mid \sigma_{1}-$ $\left.\sigma_{2}\right|_{\tilde{B}_{\rho}^{s+1}}$. Then $E$ is complete. We claim that $T$ is a contraction. Indeed

$$
d\left(T\left(\sigma_{1}\right), T\left(\sigma_{2}\right)\right)=\left|T\left(\sigma_{1}\right)-T\left(\sigma_{2}\right)\right|_{\tilde{B}_{\rho}^{s+1}} \leq\left|\partial_{\lambda} a_{n}\right|_{D_{n}^{\prime} \times \tilde{B}_{\rho}^{s+1}} d\left(\sigma_{1}, \sigma_{2}\right) \leq \frac{1}{23} d\left(\sigma_{1}, \sigma_{2}\right)
$$

as by Cauchy's estimate

$$
\left|\partial_{\lambda} a_{n}\right|_{D_{n}^{\prime} \times \tilde{B}_{\rho}^{s+1}} \leq \frac{\left|a_{n}\right|_{\Pi_{n} \times \tilde{B}_{\rho}^{s+1}}}{\operatorname{dist}\left(D_{n}^{\prime}, \partial \Pi_{n}\right)} \leq \frac{\pi / 48}{\pi / 2-\pi / 48}=\frac{1}{23} .
$$

Hence $T$ admits a unique fixed point in $E$, denoted by $\sigma_{n}$. By construction, $\sigma_{n}$ satisfies (i), (ii) and item (iii) holds since, by the uniqueness of $\sigma_{n}$ and Lemma 4.7 (ii), one has $\sigma_{n}(\bar{q},-\bar{\varphi})=\overline{\sigma_{n}(q, \varphi)}$. $\tilde{B}_{\rho}^{s+1}$,

Let $s>0$ and $\rho>0$. Then for any $|n| \geq \max \left(2 C_{0} \rho^{4}, \sqrt[s]{96 \rho^{6}}\right)$ and $(q, \varphi) \in$

$$
S_{n}\left(\sigma_{n}(q, \varphi), q, \varphi\right)=\left(\begin{array}{cc}
0 & -b_{n}^{+}\left(\sigma_{n}(q, \varphi), q, \varphi\right) \\
-b_{n}^{-}\left(\sigma_{n}(q, \varphi), q, \varphi\right) & 0
\end{array}\right) .
$$

By Lemma 4.9, we know that $b_{n}^{+}(\lambda)$ is close to $\frac{1}{4} \widehat{\varphi}(-2 n)$ and $b_{n}^{-}(\lambda)$ is close to $-\frac{1}{4} \widehat{\varphi}(2 n)$. For any given $s>0$, define the perturbed Fourier series $\mathcal{F}_{s, \rho}(q, \varphi) \in$ $H_{\mathbb{C}}^{s}$ for $(q, \varphi) \in \tilde{B}_{\rho}^{s+1}$ as follows:

$$
\begin{aligned}
\mathcal{F}_{s, \rho}(q, \varphi):= & \sum_{|n| \leq M_{s, \rho}+1} \varphi_{n} e_{n} \\
& +\sum_{n>M_{s, \rho}+1} 4 b_{n}^{+}\left(\sigma_{n}(q, \varphi), q, \varphi\right) e_{-2 n}-4 b_{n}^{-}\left(\sigma_{n}(q, \varphi), q, \varphi\right) e_{2 n}
\end{aligned}
$$

where

$$
M_{s, \rho}:=\max \left(2 C_{0} \rho^{4}, \sqrt[s]{96 \rho^{6}}, 96 \rho^{6}, 2^{20+2 s} \rho^{10}\right) .
$$

The choice of $M_{s, \rho}$ ensures that $\mathcal{F}_{s, \rho}$ is a local diffeomorphism (see proof of Lemma 4.14 below). Furthermore we introduce

$$
\Phi_{s, \rho}: \tilde{B}_{\rho}^{s+1} \rightarrow \tilde{H}_{c}^{s+1},(q, \varphi) \mapsto\left(q, \mathcal{F}_{s, \rho}(q, \varphi)\right) .
$$

Lemma 4.14. Let $\rho \geq 1$ and $s>0$. Then $\Phi_{s, \rho}: \tilde{B}_{\rho}^{s+1} \rightarrow \Phi_{\rho}^{s}\left(\tilde{B}_{\rho}^{s+1}\right) \subset \tilde{H}_{c}^{s+1}$ is a real analytic diffeomorphism such that

$$
\frac{\|\varphi\|_{s}}{2} \leq\left\|\mathcal{F}_{s, \rho}(q, \varphi)\right\|_{s} \leq 2\|\varphi\|_{s}, \quad(q, \varphi) \in \tilde{B}_{\rho}^{s+1}
$$


implying that $\tilde{B}_{\rho / 2}^{s+1} \subset \Phi_{s, \rho}\left(\tilde{B}_{\rho}^{s+1}\right)$. Moreover

$$
\sup _{(q, \varphi) \in \tilde{B}_{\rho}^{s}}\left\|\partial_{\varphi} \mathcal{F}_{s, \rho}(q, \varphi)-I d_{H_{\mathbb{C}}^{s}}\right\|_{s} \leq \frac{1}{4}
$$

Proof. For any $|n| \geq M_{s, \rho} \geq 2 C_{0} \rho^{4}, \sigma_{n}$ maps $\tilde{B}_{2 \rho}^{s+1}$ into $\Pi_{n}$ (cf. Lemma 4.13 (ii)) and $b_{n}^{ \pm}\left(\sigma_{n}(q, \varphi), q, \varphi\right)$ is well defined for $(q, \varphi) \in \tilde{B}_{2 \rho}^{s+1}$. By $(4.54)$,

$$
\begin{aligned}
\langle n\rangle^{s}\left|4 b_{n}^{+}\left(\sigma_{n}(q, \varphi), q, \varphi\right)-\widehat{\varphi}(-2 n)\right|_{\tilde{B}_{2 \rho}^{s}} & \leq\langle n\rangle^{s}\left|4 b_{n}^{+}-\widehat{\varphi}(-2 n)\right|_{\Pi_{n} \times \tilde{B}_{2 \rho}^{s}} \\
& \leq \frac{2}{|n|}(2 \rho)^{6}, \\
\langle n\rangle^{s}\left|4 b_{n}^{-}\left(\sigma_{n}(q, \varphi), q, \varphi\right)+\widehat{\varphi}(2 n)\right|_{\tilde{B}_{2 \rho}^{s}} & \leq\langle n\rangle^{s}\left|4 b_{n}^{-}+\widehat{\varphi}(2 n)\right|_{\Pi_{n} \times \tilde{B}_{2 \rho}^{s}} \\
& \leq \frac{2}{|n|}(2 \rho)^{6} .
\end{aligned}
$$

Hence the map $\mathcal{F}_{s, \rho}$ is defined on $\tilde{B}_{2 \rho}^{s+1}$ and takes values in $H^{s}(\mathbb{T}, \mathbb{C})$. Moreover, by the definition of $\mathcal{F}_{s, \rho}$ and (4.65), (4.66),

$$
\begin{aligned}
\sup _{(q, \varphi) \in \tilde{B}_{2 \rho}^{s+1}}\left\|\mathcal{F}_{s, \rho}(q, \varphi)-\varphi\right\|_{s}^{2} \leq & \sum_{n>M_{s, \rho}+1}\langle 2 n\rangle^{2 s}\left|4 b_{n}^{+}(\sigma(q, \varphi), q, \varphi)-\widehat{\varphi}(-2 n)\right|_{\tilde{B}_{2 \rho}^{s+1}}^{2} \\
& +\langle 2 n\rangle^{2 s}\left|4 b_{n}^{-}(\sigma(q, \varphi), q, \varphi)+\widehat{\varphi}(2 n)\right|_{\tilde{B}_{2 \rho}^{s+1}}^{2} \\
\leq & 2^{2 s} \sum_{n>M_{s, \rho}+1} \frac{8}{n^{2}}(2 \rho)^{12} \leq 2^{16+2 s} \frac{\rho^{12}}{M_{s, \rho}} \leq \frac{\rho^{2}}{16}
\end{aligned}
$$

By Cauchy's estimate applied to $\mathcal{F}_{s, \rho}(q, \cdot)$ on $\tilde{B}_{\rho}^{s+1}$

$$
\sup _{(q, \varphi) \in \tilde{B}_{\rho}^{s+1}}\left\|\partial_{\varphi} \mathcal{F}_{s, \rho}(q, \varphi)-I d_{H_{\mathbb{C}}^{s}}\right\|_{s} \leq \frac{1}{\rho} \sup _{(q, \varphi) \in \tilde{B}_{2 \rho}^{s+1}}\left\|\mathcal{F}_{s, \rho}(q, \varphi)-\varphi\right\|_{s} \leq \frac{1}{4}
$$

Hence $d_{\varphi} \mathcal{F}_{s, \rho}(q, \varphi): H^{s}(\mathbb{T}, \mathbb{C}) \rightarrow H^{s}(\mathbb{T}, \mathbb{C})$ is invertible for any $(q, \varphi) \in \tilde{B}_{\rho}^{s+1}$ and so is

$$
d \Phi_{s, \rho}=\left(\begin{array}{cc}
I d & 0 \\
\partial_{q} \mathcal{F}_{s, \rho} & \partial_{\varphi} \mathcal{F}_{s, \rho}
\end{array}\right) .
$$

We thus have proved that for any $s>0$, the map $\Phi_{s, \rho}: \tilde{B}_{\rho}^{s+1} \rightarrow \tilde{H}_{c}^{s+1}$ is a local diffeomorphism. Furthermore,

$$
\begin{aligned}
\left|\left\|\mathcal{F}_{s, \rho}(q, \varphi)\right\|_{s}-\|\varphi\|_{s}\right| & \leq\left\|\mathcal{F}_{s, \rho}(q, \varphi)-\varphi\right\|_{s} \\
& \leq \sup _{(q, \varphi) \in \tilde{B}_{\rho}^{s+1}}\left\|\partial_{\varphi} \mathcal{F}_{s, \rho}(q, \varphi)-I d_{H^{s}(\mathbb{T}, \mathbb{C})}\right\|_{s}\|\varphi\|<\frac{1}{4}\|\varphi\| .
\end{aligned}
$$

Hence

$$
\frac{\|\varphi\|_{s}}{2} \leq\left\|\mathcal{F}_{s, \rho}(q, \varphi)\right\|_{s} \leq 2\|\varphi\|_{s}, \quad \forall(q, \varphi) \in \tilde{B}_{\rho}^{s+1}
$$


To see that $\Phi_{s, \rho}: \tilde{B}_{\rho}^{s+1} \rightarrow \tilde{H}_{c}^{s+1}$ is one-to-one, note that for $\left(q, \varphi_{1}\right),\left(q, \varphi_{2}\right) \in$ $\tilde{B}_{\rho}^{s+1}$

$$
\begin{aligned}
\| \mathcal{F}_{s, \rho}\left(q, \varphi_{1}\right)- & \mathcal{F}_{s, \rho}\left(q, \varphi_{2}\right)-\left(\varphi_{1}-\varphi_{2}\right) \|_{s} \\
& \leq \sup _{(q, \varphi) \in \tilde{B}_{\rho}^{s+1}}\left\|\partial_{\varphi} \mathcal{F}_{s, \rho}-I d_{H^{s}(\mathbb{T}, \mathbb{C})}\right\|\left\|\varphi_{1}-\varphi_{2}\right\|_{s} \leq \frac{1}{4}\left\|\varphi_{1}-\varphi_{2}\right\|_{s} .
\end{aligned}
$$

Thus if $\mathcal{F}_{s, \rho}\left(q, \varphi_{1}\right)=\mathcal{F}_{s, \rho}\left(q, \varphi_{2}\right)$, one has $\left\|\varphi_{1}-\varphi_{2}\right\|_{s} \leq \frac{1}{4}\left\|\varphi_{1}-\varphi_{2}\right\|$ which implies $\varphi_{1}=\varphi_{2}$.

Finally, we come to the proof of Theorem 1.2. As already mentioned, we actually prove a slightly stronger version of this theorem, stated below. Recall that a potential $(q, p) \in H_{c}^{1}$ is said to be a right [left] sided $N$-gap potential with $N \in \mathbb{Z}_{\geq 0}$ if

$$
\forall n>N \quad \gamma_{n}(q, p)=0 \quad\left[\forall n>N \quad \gamma_{-n}(q, p)=0\right] .
$$

It is said to be a right [left] sided finite gap potential if it is a right [left] sided $N$-gap potential for some $N \in \mathbb{Z}$. Denote by $L F G_{c}^{s}$ and $R F G_{c}^{s}$ the following subsets of $H_{c}^{s}$ :

$$
L F G_{c}^{s}:=\left\{(q, p) \in H_{c}^{s}:(q, p) \text { left sided finite gap potential }\right\}
$$

and

$$
R F G_{c}^{s}:=\left\{(q, p) \in H_{c}^{s}:(q, p) \text { right sided finite gap potential }\right\} .
$$

\section{Theorem 4.15.}

(i) For any $s \in \mathbb{R}_{\geq 1}, L F G_{c}^{s}$ and $R F G_{c}^{s}$ are dense in $H_{c}^{s}$.

(ii) For any $s \in \mathbb{R}_{\geq 1}$, the sets $L F G_{c}^{s} \cap H_{r}^{s}$ and $R F G_{c}^{s} \cap H_{r}^{s}$ are dense in the real Hilbert space $H_{r}^{s}:=\left\{v \in H_{c}^{s}: v\right.$ real valued $\}$.

Proof. (i) Since $\tilde{H}_{c}^{s+1}$ and $H_{c}^{s+1}$ are isomorphic (cf. (4.7)) and $\tilde{H}_{c}^{s+1}$ is dense in $\tilde{H}_{c}^{s}$, it suffices to prove that, for any $s \in \mathbb{R}_{\geq 1}$ and $\rho \geq 1$, the sets

$$
\mathcal{L}_{\rho}^{s+1}:=\left\{(q, \varphi) \in \tilde{B}_{\rho}^{s+1}:(q, \varphi) \text { left sided finite gap potential }\right\}
$$

and

$$
\mathcal{R}_{\rho}^{s+1}:=\left\{(q, \varphi) \in \tilde{B}_{\rho}^{s+1}:(q, \varphi) \text { right sided finite gap potential }\right\}
$$

are dense in $\tilde{B}_{\rho}^{s+1}$. By a slight abuse of terminology, we say that $(q, \varphi)=(q, P p+$ $\left.q_{x}\right)$ is a right or left sided finite gap potential if $(q, p)$ is such a potential. Let us first prove that $\mathcal{R}_{\rho}^{s+1}$ is dense in $\tilde{B}_{\rho}^{s+1}$. For any $M \in \mathbb{Z}_{\geq 1}$, denote by $\mathcal{G}_{s, M}$ the closed subspace of $H^{s}(\mathbb{T}, \mathbb{C})$ spanned by $e_{2 k}=e^{\mathrm{i} 2 k \pi x},|k| \leq M$. Then $\mathcal{G}_{s, M}$ is an increasing sequence of subspaces of $H^{s}(\mathbb{T}, \mathbb{C})$ and $\bigcup_{M \geq M_{0}} \mathcal{G}_{s, M}$ is dense in $H^{s}(\mathbb{T}, \mathbb{C})$. Hence $\bigcup_{M \geq M_{0}} H^{s+1}(\mathbb{T}, \mathbb{C}) \times \mathcal{G}_{s, M}$ is dense in $\tilde{H}_{c}^{s+1}$. Here $M_{0}=$ $\max \left(M_{s, \rho}, N_{1}\right)$, where $M_{s, \rho}$ is given by (4.63) and $N_{1}$ by Theorem 4.10. Since, by Lemma $4.14, \Phi_{s, \rho}: \tilde{B}_{\rho}^{s+1} \rightarrow \Phi_{s, \rho}\left(\tilde{B}_{\rho}^{s+1}\right)$ is a (real analytic) diffeomorphism, it 
follows that the preimage of $\Phi_{s, \rho}\left(\tilde{B}_{\rho}^{s+1}\right) \cap\left(\bigcup_{M \geq M_{0}} H^{s+1}(\mathbb{T}, \mathbb{C}) \times \mathcal{G}_{s, M}\right)$ is dense in $\tilde{B}_{\rho}^{s+1}$. We claim that any element in this set is a right sided finite gap potential. Indeed, if for any given $(q, \varphi) \in \tilde{B}_{\rho}^{s+1}, \Phi_{s, \rho}(q, \varphi)=\left(q, \mathcal{F}_{s, \rho}(q, \varphi)\right)$ is in $H^{s+1}(\mathbb{T}, \mathbb{C}) \times \mathcal{G}_{s, M}$ for some $M \geq M_{0}$, then by the definition (4.63) of $\mathcal{F}_{s, \rho}$, $b_{n}^{-}\left(\sigma_{n}(q, \varphi), q, \varphi\right)=0$ and $b_{n}^{+}\left(\sigma_{n}(q, \varphi), q, \varphi\right)=0$ for any $n>M$. Since $(q, \varphi) \in$ $\tilde{B}_{\rho}^{s+1}$ and $M \geq M_{s, \rho} . S_{n}(\lambda, q, \varphi)$ is well defined for $\lambda \in \Pi_{n}$ (cf. (4.42)). Since $M \geq N_{1}$, it follows from Theorem 4.10 that

$$
\operatorname{det} S_{n}\left(\sigma_{n}(q, \varphi)\right)=\left(\sigma_{n}(q, \varphi)-\pi n-a_{n}\left(\sigma_{n}(q, \varphi)\right)\right)^{2}-\left.b_{n}^{+} b_{n}^{-}\right|_{\sigma_{n}(q, \varphi)}=0
$$

Note that $\sigma_{n}(q, \varphi)$ is a double root of $\left(\sigma_{n}(q, \varphi)-\pi n-a_{n}\left(\sigma_{n}(q, \varphi)\right)\right)^{2}$ as well as a double root of $b_{n}^{+} b_{n}^{-}$hence it is a double root of $\operatorname{det} S_{n}$ in $\Pi_{n}$, implying that $\gamma_{n}(q, \varphi)=0$. It means that $(q, \varphi)$ is a right sided $M$-gap potential. We thus have shown that $\mathcal{R}_{\rho}^{s+1}$ is dense in $\tilde{B}_{\rho}^{s+1}$. Using Lemma 2.14 (reciprocity in $\lambda$ ), one sees that the arguments above applied to $(-q, \varphi)$ yield that $\mathcal{L}_{\rho}^{s+1}$ is also dense in $\tilde{B}_{\rho}^{s+1}$. This proves (i). Item (ii) is proved in the same way.

Supports. Both authors were supported by the Swiss National Science Foundation.

\section{References}

[1] P. Djakov and B. Mityagin, Instability zones of periodic 1-dimensional Schrödinger and Dirac operators, Russian Math. Surveys 61 (2006), No. 4, 663-766.

[2] V.E. Zaharov, L.A. Tahtadžjan, and L.D. Faddeev, A complete description of the solutions of the "sine-Gordon" equation, Dokl. Akad. Nauk SSSR 219 (1974), 13341337 (Russian).

[3] B. Grébert and T. Kappeler, The Defocusing NLS Equation and Its Normal Form, Lectures in Mathematics, European Mathematical Society, Zürich, 2014.

[4] T. Kappeler and B. Mityagin, Estimates for periodic and Dirichlet eigenvalues of the Schrödinger operator, SIAM J. Math. Anal. 33 (2001), No. 1, 113-152.

[5] T. Kappeler and J. Pöschel, KdV \& KAM, Ergebnisse der Mathematik und ihrer Grenzgebiete, Springer Verlag, New York, 2003.

[6] T. Kappeler, F. Serier, and P. Topalov, On the characterization of the smoothness of skew-adjoint potentials in periodic Dirac operators, J. Funct. Anal. 256 (2009), No. 7, 2069-2112.

[7] V. Marchenko, Sturm-Liouville Operators and Applications, Operator Theory: Advances and Applications, vol. 22, Birkhäuser, Basel, 1986.

[8] H. McKean, The sine-Gordon and sinh-Gordon equations on the circle, Comm. Pure Appl. Math. 34 (1981), No. 2, 197-257.

[9] J. Pöschel, Hill's potentials in weighted Sobolev spaces and their spectral gaps, Math. Ann. 349 (2011), No. 2, 433-458. 
Received February 15, 2018.

Thomas Kappeler,

Institut für Mathematik, Universität Zürich, Winterthurerstrasse 190, CH-8057 Zürich, Switzerland,

E-mail: thomas .kappeler@math.uzh.ch

Yannick Widmer,

Institut für Mathematik, Universität Zürich, Winterthurerstrasse 190, CH-8057 Zürich, Switzerland,

E-mail: Yannick.Widmer@math.uzh.ch

\section{Про спектральні властивості оператора $L$ у парі Лакса рівняння sine-Гордона}

Thomas Kappeler and Yannick Widmer

Ми аналізуємо періодичний спектр оператора $L$ у парі Лакса рівняння sine-Гордона в термінах регулярності потенціалу.

Ключові слова: рівняння sine-Гордона, рівняння sinh-Гордона, пара Лакса, властивості спадання довжин лакун. 